\title{
Data report: dissolved minor element compositions, sediment major and minor element concentrations, and reactive iron and manganese data from the Lesser Antilles volcanic arc region, IODP Expedition 340 Sites U1394, U1395, U1396, U1399, and U1400'
}

Natalie A. Murray, ${ }^{2}$ Jesse M. Muratli, ${ }^{3}$ Anne M. Hartwell, ${ }^{2}$ Hayley Manners, ${ }^{4}$ Meghan R. Megowan, ${ }^{3}$ Miguel Goñi, ${ }^{3}$ Martin Palmer, ${ }^{5}$ and James McManus ${ }^{2,} 6$

\section{Chapter contents}

Abstract....................

Introduction $\ldots \ldots \ldots \ldots \ldots \ldots \ldots \ldots$

Analytical methods ...............

Results..................... 4

Acknowledgments.............. 5

References..................

Figures....................

Tables. ...................... 18

1 Murray, N.A., Muratli, J.M., Hartwell, A.M., Manners, H., Megowan, M.R., Goñi, M., Palmer, M., and McManus, J., 2016. Data report: dissolved minor element compositions, sediment major and minor element concentrations, and reactive iron and manganese data from the Lesser Antilles volcanic arc region, IODP Expedition 340 Sites U1394, U1395, U1396, U1399, and U1400. In Le Friant, A., Ishizuka, O., Stroncik, N.A., and the Expedition 340 Scientists, Proceedings of the Integrated Ocean Drilling Program, 340: Tokyo (Integrated Ocean Drilling Program Management International, Inc.).

doi:10.2204/iodp.proc.340.207.2016

${ }^{2}$ The University of Akron, Department of Geosciences, Akron OH 44235, USA.

${ }^{3}$ Oregon State University, College of Earth, Ocean, and Atmospheric Sciences, Corvallis OR 97331, USA.

${ }^{4}$ University of Plymouth, School of Geography, Earth and Environmental Sciences, Plymouth, Devon PL4 8AA, United Kingdom.

${ }^{5}$ National Oceanography Centre Southampton, Southampton SO14 3ZH, United Kingdom. ${ }^{6}$ Present address: Bigelow Laboratory for Ocean Sciences, 60 Bigelow Drive, P.O. Box 380, East Boothbay ME 04544, USA. Correspondence author: jmcmanus@bigelow.org

\section{Abstract}

We measured dithionite-extractable iron and manganese along with a variety of bulk sedimentary solid and dissolved phases to constrain diagenetic reactions occurring within the sediment package of Grenada Basin, which is within the Lesser Antilles volcanic arc region. Core material was obtained during Integrated Ocean Drilling Program Expedition 340. For this report we focus primarily on five sites; three sites are located in the northern portion of the study area off the island of Montserrat (Sites U1394U1396), and two sites are located farther south off the island of Martinique (Sites U1399 and U1400). Sediments throughout this region include tephra-rich volcanic sands, hemipelagic mud sequences, and carbonate-rich sequences, with widely variable proportions over short (centimeter scale) depth intervals. Regardless of the main sediment type, organic carbon contents are low with average values of $0.19 \pm 0.1 \mathrm{wt} \%$ at Site U1394, $0.13 \pm 0.08 \mathrm{wt} \%$ at Site U1395, $0.13 \pm 0.06 \mathrm{wt} \%$ at Site U1396, $0.28 \pm 0.08 \mathrm{wt} \%$ at Site U1399, and $0.23 \pm 0.15 \mathrm{wt} \%$ at Site U1400. Carbonate contents are more variable, ranging between 0 and $~ 80 \mathrm{wt} \%$, in cores from Sites U1394-U1396 and between 1 and $40 \mathrm{wt} \%$ at both Sites U1399 and U1400. These variations in carbonate content likely reflect variable dilution with volcanogenic sediment. Pore fluids reflect a range of diagenetic conditions from oxidizing (sulfate rich) to sulfate-reducing conditions. Reactive major elements $(\mathrm{Ca}$ and $\mathrm{Mg}$ ) as well as a number of minor elements show a range of diagenetic behaviors from reactions likely related to carbonate dissolution or precipitation to exchange reactions between pore fluids and the volcanic-rich sedimentary substrate and clay formation. However, significant site-to-site variability is seen in the diagenetic behavior of these elements. Solid-phase reactive Fe ranges from 0.18 to $0.75 \mathrm{wt} \%$ at the northern sites and 0.4 to 1.5 $\mathrm{wt} \%$ at the southern sites, whereas reactive $\mathrm{Mn}$ ranges from 0 to $0.1 \mathrm{wt} \%$ in the north and 0 to $0.3 \mathrm{wt} \%$ in the south.

\section{Introduction}

Integrated Ocean Drilling Program (IODP) Expedition 340 cored several sites within the Lesser Antilles volcanic arc region. The 
overall goal of the project was to drill volcanogenic landslides in the marine environment to establish a record of eruption cycles and the impact of these landslides on the marine sedimentary record. This report focuses on sediment geochemical results from five sites (U1394-U1396, U1399, and U1400; Fig. F1). Sites U1394-U1396 are located south of the island of Montserrat in 1115, 1191, and $801 \mathrm{~m}$ of water, respectively (see the "Expedition 340 summary" chapter [Expedition 340 Scientists, 2013a]). Site U1394 is located $24 \mathrm{~km}$ southeast of Montserrat, whereas Site U1395 is located further southeast of Site U1394, between the islands of Montserrat and Guadeloupe (Fig. F1). The lithology of Sites U1394 and U1395 is similar and consists of hemipelagic muds, turbidite deposits, mafic volcaniclastics and volcaniclastic sands, hemipelagic mud, and tephra layers. Although Site U1396 to the west has similar sedimentation to Sites U1394 and U1395, given that this site resides on a bathymetric high our expectation is that the sediments will have a tendency toward more fine grained material; nevertheless, volcaniclastic sand, hemipelagic mud, and tephra layers all dominate this core's lithology as well (see the "Expedition 340 summary" chapter [Expedition 340 Scientists, 2013a]); Le Friant et al., 2015; Palmer et al., 2016).

Sites U1399 and U1400 are located west of the island of Martinique in Grenada Basin in 2900 and $2745 \mathrm{~m}$ water depth, respectively (Fig. F1). The lithology of these two sites is similar and is composed of hemipelagic mud, interbedded tephra layers, volcaniclastic turbidites, and deformed sediments (see the "Expedition 340 summary" chapter [Expedition 340 Scientists, 2013a]); Le Friant et al., 2015). Site U1400 is located west of Martinique but closer in proximity to the island than Site U1399 (Fig. F1).

\section{Analytical methods}

\section{Pore fluid extractions}

Pore fluids were obtained from all sediment cores presented in this report (Table T1). Sampling occurred every $10 \mathrm{~m}$ unless the sediment was unsuitable for pore fluid extraction (i.e., because of sands, debris, or other mission-specific rationales). Sampling procedures are described in detail in the "Methods" chapter (Expedition 340 Scientists, 2013b); however, we present a brief description for completeness. A 10 to $15 \mathrm{~cm}$ section of whole-round core was removed to begin the squeezing process in the laboratory. Whole-round sections were processed within a nitrogen-filled bag at room temperature and then transferred to a hydraulic press for pore fluid extraction (Manheim, 1966). Following extraction, pore fluids were filtered and subsampled for various dissolved constituents (see the "Methods" chapter [Expedition 340 Scientists, 2013b]).

\section{Pore fluid ICP-OES and ICP-MS methods}

As part of this report, we provide some previously published major element data $\left(\mathrm{Ca}, \mathrm{Mg}\right.$, and $\left.\mathrm{SO}_{4}{ }^{2-}\right)$ (see the "Expedition 340 summary" and "Methods" chapters [Expedition 340 Scientists, 2013a, 2013b]) along with previously unpublished minor element data ( $\mathrm{Li}, \mathrm{B}, \mathrm{Sr}, \mathrm{Si}, \mathrm{Mn}, \mathrm{Rb}$, and Cs; Table T1). For the minor elements ( $\mathrm{Li}, \mathrm{B}, \mathrm{Si}$, and $\mathrm{Mn})$, samples were diluted to a $0.25: 5$ ratio with $1 \%$ quartz-distilled nitric acid. Strontium was measured twice, once with the major elements at 100-fold dilution and once with the minor elements at 20-fold dilution. Together, these two runs capture the full dynamic range of $\mathrm{Sr}$ in these samples with greater precision than either single analysis alone. Minor element standards were matrix-matched to the samples with an artificial salt water solution made to approximate seawater concentrations of $\mathrm{Na}, \mathrm{Mg}$, and $\mathrm{Cl}$ from ultrapure salts (Sigma Aldrich). Instrumental drift was monitored by repeated runs of the standard curve, but was not found to influence the inductively coupled plasma-optical emissions spectrometer (ICP-OES) results significantly. Individual sample values represent a mean of three replicate analyses for each element in each sample tube. Uncertainties reported here are $1 \sigma$ uncertainties derived from two sources: (1) the regression uncertainty, calculated using the standard error of the regression, and (2) the "internal" uncertainty calculated from the standard deviation of the three replicate analyses. The reported uncertainties are combined as the square root of the sum of squares. Detection limits reported here are the point at which the regression uncertainty is one-third of the concentration. Above this point, the concentration measured is $>3 \sigma$ above zero.

$\mathrm{Cs}$ and $\mathrm{Rb}$ samples were run on a Thermo X-Series II inductively coupled plasma-mass spectrometer (ICPMS). ICP-MS signals can drift significantly over the course of an analytical session; therefore, instrumental drift was corrected by spiking each sample tube with an internal standard solution. This solution consisted of $\sim 50 \mathrm{ppb}$ each of Rh and Re. Measured counts throughout the run were corrected to the value of the first acid blank run of the day. Although Re was not used to correct either of the target elements, it was used as an assessment tool to monitor instrumental drift. Samples were diluted to a 0.25:5 ratio with $1 \%$ quartz-distilled nitric acid. Standards were spiked with the same artificial salt water solution as used for the minor element analysis to match the matrix of the samples. Though ultrapure salts 
were used, we found this solution contained a Cs blank. We determined that the solution contained $\sim 2.5 \pm 0.1 \mathrm{nmol} / \mathrm{L}$ in the undiluted salt water solution, and this value was used to correct the standard concentrations.

\section{Solid-phase total digestion methods and analyses}

Total sediment digestion methods are described in detail elsewhere (Muratli et al., 2012; Muratli et al., 2015). Briefly, prior to digestion sediments were dried and ground. Total sediment digestion was accomplished through a microwave-assisted (CEM MARS-5 microwave oven) digestion process that utilized the inorganic acids $\mathrm{HCl}, \mathrm{HNO}_{3}$, and $\mathrm{HF}(\mathrm{Mu}-$ ratli et al., 2015). Samples were diluted with 5\% $\mathrm{HNO}_{3}$ and were heated for $\sim 24 \mathrm{~h}$ prior to analysis to remove residual fluoride complexes (Muratli et al., 2012).

To assess precision, laboratory standards (PACS-2 and an in-house standard RR9702A-42MC) were digested on multiple occasions, and $\sim 10 \%$ of the samples during a run were replicated. RR9702A-42MC is an in-house Chilean margin sediment standard that our group has been using to assess long-term method precision, and PACS-2 is a marine sediment reference material (National Research Council [NRC] Canada) from the Harbour of Esquimalt, Canada.

For the solid-phase major element data (Table T2), we analyzed samples on a Leeman Laboratories Prodigy ICP-OES at the K.W. Keck Collaboratory at Oregon State University (USA), and our approach was similar to that employed for pore fluids as discussed above. Specific emission wavelengths for analysis of solid-phase digests are reported in Table T3. Samples and standards were typically diluted 20 -fold with $1 \%$ quartz-distilled nitric acid. For elements affected by instrumental drift, a correction was applied. Data values are an average of three replicate analyses for each element. We report uncertainties at $1 \sigma$, which are calculated as indicated for the dissolved phases.

\section{Dithionite-extractable Fe and Mn}

A single-step mild chemical leach was performed on samples to extract the labile or "reactive" fraction of Fe and Mn from the sediments (Mehra and Jackson, 1960) (Tables T4, T5, T6). We added $\sim 0.25 \mathrm{~g}$ of dried ground sediment to centrifuge tubes and then added $\sim 10 \mathrm{~mL}$ of dithionite reagent. We used a sodium acetate, sodium citrate solution as a buffer (Kostka and Luther, 1994; Roy et al., 2013). Centrifuge tubes were agitated with a vortex stirrer and placed in a heating block for $4 \mathrm{~h}$ at $60^{\circ} \mathrm{C}$. Each sample was mixed every $15 \mathrm{~min}$, and at the end of $4 \mathrm{~h}$ samples were centri- fuged at $4000 \mathrm{rpm}$ for $5 \mathrm{~min}$. Leachate was then transferred into a labeled bottle, and sample mass was recorded. For some of the samples (Sites U1399 and U1400), a precipitate or gel-like substance precipitated from solution over time. Samples that showed the presence of a precipitate were reextracted and then diluted immediately following extraction.

Analyses for reactive $\mathrm{Fe}$ and $\mathrm{Mn}$ samples (Sites U1394-U1396) were analyzed on a Leeman Labs Prodigy ICP-OES at Oregon State University (Table T4), and samples from Sites U1399 and U1400 were analyzed on a Perkin Elmer Atomic Absorption Spectrometer, AAnalyst 700, at the University of Akron (Tables T4, T5). Additional samples for Site U1396 were also analyzed at the University of Akron on an Agilient Technologies 700 series ICP-OES (Table T6). For the samples from Sites U1394-U1396 that were measured at Oregon State University, we assessed precision by using the standard reference material, PACS-2 (NRC Canada), as well as our Chilean margin laboratory standard. Reactive Fe in these two standards was measured to be $0.79 \pm 0.02 \mathrm{wt} \%$ for the PACS-2 standard and $0.93 \pm 0.02 \mathrm{wt} \%$ for the Chilean margin standard. For reactive $\mathrm{Mn}$, the values are $0.0029 \pm 0.0001 \mathrm{wt} \%$ and $0.0024 \pm 0.0001 \mathrm{wt} \%$, respectively. These values compare favorably with previous runs from our laboratory with the exception that the reactive iron values are slightly lower than the longer term average reported in Roy et al. (2013), where the reactive Fe values for PACS- 2 and Chilean margin samples are $0.88 \pm 0.08 \mathrm{wt} \%$ and $1.08 \pm 0.08$ $\mathrm{wt} \%$, respectively. The values reported for this communication are, however, consistent with those reported in Muratli et al. (2015). To assess precision for the samples from Sites U1399 and U1400 (Tables T4, T5), we used an in-house carbonate-rich laboratory standard, PACS-3 (NRC Canada), and the standard reference material 2702 (National Institute of Standards and Technology), which is a marine sediment from Baltimore Harbor. Reactive Fe for these samples was determined to be $0.71 \% \pm 0.03 \%$ (in-house carbonate), $0.85 \% \pm 0.04 \%$ (PACS-3), and $3.11 \% \pm$ $0.08 \%$ (2702), and reactive $\mathrm{Mn}$ is $0.16 \% \pm 0.05 \%$ (inhouse carbonate) and $0.15 \% \pm 0.05 \%$ (2702). Reactive Mn for PACS-3 was not detectable.

Reproducibility for samples (Table T6) was accessed from standard reference materials PACS-3, MESS-3, and MESS- 4 that were analyzed during extractions with unknown samples. Iron, manganese, and aluminum in PACS-3 are $0.89 \% \pm 0.03 \%(N=5)$, $0.0038 \% \pm 0.0008 \%(N=5)$, and $0.121 \% \pm 0.010 \%$ $(\mathrm{N}=3)$, respectively. Fe, $\mathrm{Mn}$, and Al for MESS-3 are $1.68 \% \pm 0.05 \%(N=5), 0.0182 \% \pm 0.0009 \%(N=5)$, and $0.131 \% \pm 0.001 \%(N=3)$, respectively. Fe, Mn, 
and $\mathrm{Al}$ in MESS-4 are $1.51 \% \pm 0.07 \%(N=3)$, $0.0171 \% \pm 0.0003 \%(N=3)$, and $0.143 \% \pm 0.001 \%$ $(N=3)$, respectively.

\section{Organic carbon, nitrogen, and inorganic carbon}

Organic carbon and nitrogen for most of the samples presented here were analyzed at Oregon State University following the methodology outlined in Goñi et al. (2003) with minor modifications (Tables T4, T5). This technique uses $20-30 \mathrm{mg}$ of sample placed into silver boats and exposed to hydrochloric vapors to remove all inorganic carbon (Hedges and Stern, 1984). Complete removal of inorganic carbon involves pipetting one to two drops of $10 \% \mathrm{HCl}$ into the samples after vapor acidification is complete. Samples are then dried and run on a Thermo Quest EA2500 elemental analyzer. Blanks containing minor amounts of carbon but no nitrogen were analyzed. Organic carbon measurements were corrected for the amount of carbon recorded by the blanks. Inorganic carbon measurements were completed at The University of Akron on a UIC Coulometrics coulometer. The analysis required $\sim 40 \mathrm{mg}$ of sample placed in gelatin capsules. A purge of the sample flask with $\mathrm{CO}_{2}$-free carrier gas removes atmospheric $\mathrm{CO}_{2}$. Following the purge, $5 \mathrm{~mL}$ of perchloric acid $\left(\mathrm{HClO}_{4}\right)$ was added to the flask to acidify the inorganic carbon in order to determine the amount of $\mathrm{CO}_{2}$ within the sample.

Hole U1396C tephra samples (Table T6) were freezedried $(24 \mathrm{~h})$, crushed using a granite pestle and mortar, and decarbonated using excess hydrochloric acid $(10 \% \mathrm{v} / \mathrm{v})$ until any visible sign of reaction had ceased. This process was followed by repeated washing with deionized water until a neutral solution was obtained, followed by freeze drying $(24 \mathrm{~h})$. Approximately $10 \mathrm{mg}$ of decarbonated and unaltered sample was weighed into a tin cup and then was crushed and analyzed for total organic carbon (TOC) and total carbon (TC) content, respectively, using a Carlo Erba 1110 elemental analyzer with L-cysteine as the calibration standard.

\section{Results}

\section{Pore fluids}

Some pore fluid results from the expedition have been presented previously (see the "Expedition 340 summary" chapter [Expedition 340 Scientists, 2013a]), and this report contributes additional data as discussed above. Both the major (see the "Expedition 340 summary" chapter [Expedition 340 Scientists, 2013a]) and minor element data (Table T1; Figs.
F2, F3, F4, F5, F6, F7) show a variety of distributions that are likely influenced by a mixture of reactions that include organic carbon decomposition, carbonate dissolution and precipitation reactions, and volcanogenic sediment diagenesis. $\mathrm{Ca}$ and $\mathrm{Mg}$ are two of the more diagnostic elements for differentiating among these processes. For example, data from Site U1396 demonstrates Ca increases that mirror the $\mathrm{Mg}$ decreases (Fig. F4), which is quite likely driven by exchange reactions during diagenesis of volcanic glass (see the "Expedition 340 summary" chapter [Expedition 340 Scientists, 2013a]). By contrast, Site U1399 shows a decrease in $\mathrm{Ca}$ and $\mathrm{Mg}$, which is likely a result, at least in part, of carbonate precipitation reactions at depth (Fig. F5). Sulfate varies from being quite close to the seawater value $(\sim 28 \mathrm{mM})$ throughout the profile (Site U1396; Fig. F4) to being depleted to $\sim 10 \mathrm{mM}$ at Site U1394 (Fig. F2). Manganese exhibits a variety of behaviors likely reflecting organic carbon oxidation reactions as well as variations in sedimentary reactive Mn content. The other elements, $\mathrm{Si}, \mathrm{B}, \mathrm{Li}, \mathrm{Sr}, \mathrm{Rb}$, and $\mathrm{Cs}$, all show a similar diversity in behaviors that are likely driven by these same or similar diagenetic reactions involving volcanic material, carbonate, biogenic silica, and organic matter (Figs. F2, F3, F4, F5, F6).

\section{Whole-sediment digestion results}

Whole-sediment digestion results are presented in Table T2. These results are for selected samples from Sites U1395, U1396, U1399, and U1400 and are not plotted separately, as there are too few data to construct meaningful interpretations. However, results do show considerable variability, which is consistent with the highly variable mixture of hemipelagic muds, turbidites, and volcaniclastic sediments.

\section{Dithionite extraction results}

Whole-core distributions

Dithionite-extractable iron $\left(\mathrm{Fe}_{\mathrm{R}}\right)$ and manganese $\left(\mathrm{Mn}_{\mathrm{R}}\right)$ as well as organic carbon (OC) generally have lower concentrations at the northern sites (Figs. F7, F8, F9) with highly variable $\mathrm{CaCO}_{3}(0-81 \mathrm{wt} \%)$ as compared to the southern sites, which show more variation in their $\mathrm{Fe}_{R}, \mathrm{Mn}_{\mathrm{R}}$, and $\mathrm{OC}$ concentrations with less variation in their $\mathrm{CaCO}_{3}$ contents (Figs. F10, F11; Table T4).

At Site U1394, $\mathrm{Fe}_{\mathrm{R}}, \mathrm{Mn}_{\mathrm{R}}$, and OC have relatively low concentrations throughout the record. In the uppermost $25 \mathrm{~m}$ of sediment, these three components have average values of $0.39 \pm 0.09 \mathrm{wt} \%, 0.03 \pm 0.01$ $\mathrm{wt} \%$, and $0.19 \pm 0.1 \mathrm{wt} \%$, respectively. Carbonate varies from 5 to $75 \mathrm{wt} \%$ within the uppermost $22 \mathrm{~m}$ of sediment. An $\sim 87 \mathrm{~m}$ region produced no data, 
which reflects the fact that fine-grained material recovery within this interval was generally poor. From 111 to 197 meters below seafloor (mbsf), $\mathrm{Fe}_{R}$ and $\mathrm{Mn}_{\mathrm{R}}$ are relatively constant, averaging $0.37 \pm 0.03$ $\mathrm{wt} \%$ and $0.03 \pm 0.01 \mathrm{wt} \%$, respectively (Fig. F7A, F7B). Organic carbon averages $0.23 \pm 0.1 \mathrm{wt} \%$ (Fig. F7C), and carbonate shows less variability in its concentration (55-60 $\mathrm{wt} \%)$ compared to the upper 22 mbsf, with the exception of three low-concentration samples (Fig. F7D).

Some of the solid-phase concentrations at Site U1395 show a wider range in concentration compared to Site U1394. $\mathrm{Fe}_{\mathrm{R}}$, in particular, ranges from 0.18 to $0.75 \mathrm{wt} \%$ throughout the core with more variability below 60 mbsf (Fig. F8A). In contrast, $\mathrm{Mn}_{R}$ is particularly low, averaging $0.02 \pm 0.02 \mathrm{wt} \%$, with the exception of a single data point $(0.09 \mathrm{wt} \%)$ at $\sim 100$ mbsf (Fig. F8B). Organic carbon averages $0.13 \pm$ $0.08 \mathrm{wt} \%$ (Fig. F8C). Calcium carbonate varies between 0 and $76 \mathrm{wt} \%$, and at $\sim 169 \mathrm{mbsf}, \mathrm{CaCO}_{3}$ decreases from 81 to $43 \mathrm{wt} \%$ (Fig. F8D).

Site U1396 solid phases, with the exception of $\mathrm{CaCO}_{3}$, are relatively constant with depth (Fig. F9). $\mathrm{Fe}_{\mathrm{R}}$ averages $0.35 \pm 0.06 \mathrm{wt} \%$ with depth, with the highest sample at $0.48 \mathrm{wt} \%$ (Fig. F9A). $\mathrm{Mn}_{\mathrm{R}}$ and $\mathrm{OC}$ are both uniformly low, averaging $0.02 \pm 0.01 \mathrm{wt} \%$ and $0.13 \pm 0.06 \mathrm{wt} \%$, respectively (Fig. F9B, F9C). As is the case for Site U1395, calcium carbonate values show large variability ranging from 0 to $74 \mathrm{wt} \%$ throughout the core.

Site U1399 solid-phase data exhibit more variability in $\mathrm{Fe}_{\mathrm{R}}, \mathrm{Mn}_{\mathrm{R}}$, and $\mathrm{OC}$ with depth compared to Sites U1394-U1396 (Fig. F10). $\mathrm{Fe}_{\mathrm{R}}$ has greater variability $(0.46-1.4 \mathrm{wt} \%)$ in the uppermost $75 \mathrm{~m}$ of the core compared to the lowermost $\sim 179 \mathrm{~m}$ of the core $(0.42-1.06 \mathrm{wt} \%)$ (Fig. F10A). $\mathrm{Mn}_{\mathrm{R}}$ is highly variable compared to the northern cores and has an average of $0.065 \pm 0.046 \mathrm{wt} \%$ (Fig. F10B). Organic carbon ranges from 0.01 to $0.61 \mathrm{wt} \%$ with an average of $0.28 \pm 0.15 \mathrm{wt} \%$ (Fig. F10C). $\mathrm{CaCO}_{3}$ ranges from 2.5 to $36 \mathrm{wt} \%$ with depth, showing less variation compared to the northern sites (Fig. F10D).

Site U1400 solid-phase data are similar to those of Site U1399, with $\mathrm{Fe}_{\mathrm{R}}, \mathrm{Mn}_{\mathrm{R}}$, and, to a lesser extent, OC exhibiting more variation with depth and less variability in $\mathrm{CaCO}_{3}$ compared to the northern sites (Fig. F11). Reactive Fe ranges from 0.4 to $1.4 \mathrm{wt} \%$ with an average of $0.8 \pm 0.3 \mathrm{wt} \%$ (Fig. F11A). $\mathrm{Mn}_{R}$ values exhibit considerable scatter throughout the core, with an average value of $0.068 \pm 0.037$ wt $\%$ (Fig. F11B). Organic carbon shows a slight decrease to $200 \mathrm{mbsf}$ and then increases with depth. However, OC is less abundant at Site U1400 compared to Site U1399, ranging from 0.1 to $0.4 \mathrm{wt} \%$ with an average of 0.23 $\pm 0.08 \mathrm{wt} \%$ (Fig. F11C). Calcium carbonate ranges from 2 to $35 \mathrm{wt} \%$ and demonstrates increases at approximately the same depths as $\mathrm{Mn}_{\mathrm{R}}$ (Fig. F11D).

\section{Discrete intervals}

In addition to the results presented above, we analyzed samples from Sites U1399 and U1400 from focused or discrete sedimentary intervals (Table T5). These intervals were chosen based on abrupt lithology transitions. A total of nine different sections (56 samples in each section) were chosen, six from Site U1399 and three from Site U1400. The different sections include (in order of increasing depth) Sections 340-U1399B-3H-3, 7H-3, 11H-2, 16H-2, 19H-2, and 25H-2 and Sections 340-U1400B-16H-2, 26H-5, and 48X-1. Solid-phase data and visual lithology descriptions can be found in Table T5, and the results are plotted on Figures F12, F13, F14, F15, F16, F17, F18, F19, and F20. The general observation is that these changes in lithology often coincide with changes in reactive Fe and $\mathrm{Mn}$ as well as with $\mathrm{OC}$ and carbonate. Although organic carbon concentrations are generally low, they are typical for pelagic sedimentary environments (e.g., Ziebis et al., 2012). Concentrations of $\mathrm{OC}, \mathrm{Fe}_{\mathrm{R}}$, and $\mathrm{Mn}_{\mathrm{R}}$ are generally higher in the finer grain sediments as compared to the courser sedimentary horizons (Table T5).

Table T6 presents results for $\mathrm{Fe}, \mathrm{Mn}$, and $\mathrm{Al}$ from dithionite extractions on samples that were identified to be rich in volcanic ash/tephra. These sediments show that these layers can vary significantly in their $\mathrm{Fe}_{\mathrm{R}}$ and $\mathrm{Mn}_{\mathrm{R}}$ concentrations.

\section{Acknowledgments}

This report used samples and data provided by the Integrated Ocean Drilling Program (IODP). IODP is sponsored by the U.S. National Science Foundation (NSF). Financial support was provided by the U.S. Science Support Program (USSSP) and the US National Science Foundation under grant Number 1360077 for shore-based analyses.

\section{References}

Expedition 340 Scientists, 2013a. Expedition 340 summary. In Le Friant, A., Ishizuka, O., Stroncik, N.A., and the Expedition 340 Scientists, Proceedings of the Integrated Ocean Drilling Program, 340: Tokyo (Integrated Ocean Drilling Program Management International, Inc.). http://dx.doi.org/10.2204/ iodp.proc.340.101.2013

Expedition 340 Scientists, 2013b. Methods. In Le Friant, A., Ishizuka, O., Stroncik, N.A., and the Expedition 340 Scientists, Proceedings of the Integrated Ocean Drilling Pro- 
gram, 340: Tokyo (Integrated Ocean Drilling Program Management International, Inc.). http://dx.doi.org/ 10.2204/iodp.proc.340.102.2013

Goñi, M.A., Teixeira, M.J., and Perkey, D.W., 2003. Sources and distribution of organic matter in a river-dominated estuary (Winyah Bay, SC, USA). Estuarine, Coastal and Shelf Science, 57(5-6):1023-1048. http://dx.doi.org/ 10.1016/S0272-7714(03)00008-8

Hedges, J.I., and Stern, J.H., 1984. Carbon and nitrogen determinations of carbonate-containing solids. Limnology and Oceanography, 29(3):657-663. http:// dx.doi.org/10.4319/1o.1984.29.3.0657

Kostka, J.E., and Luther, G.W., III, 1994. Partitioning and speciation of solid phase iron in saltmarsh sediments. Geochimica et Cosmochimica Acta, 58(7):1701-1710. http://dx.doi.org/10.1016/0016-7037(94)90531-2

Le Friant, A., Ishizuka, O., Boudon, G., Palmer, M.R., Talling, P.J., Villemant, B., Adachi, T., Aljahdali, M., Breitkreuz, C., Brunet, M., Caron, B., Coussens, M., Deplus, C., Endo, D., Feuillet, N., Fraas, A.J., Fujinawa, A., Hart, M.B., Hatfield, R.B., Hornbach, M., Jutzeler, M., Kataoka, K.S., Komorowski, J.-C., Lebas, E., Lafuerza, S., Maeno, F., Manga, M., Martínez-Colon, M., McCanta, M., Morgan, S., Saito, T., Slagle, A., Sparks, S., Stinton, A., Stroncik, N., Subramanyam, K.S.V., Tamura, Y., Trofimovs, J., Voight, B., Wall-Palmer, D., Wang, F., and Watt, S.F.L., 2015. Submarine record of volcanic island construction and collapse in the Lesser Antilles arc: first scientific drilling of submarine volcanic island landslides by IODP Expedition 340. Geochemistry, Geophysics, Geosystems, 16(2):420-442. http://dx.doi.org/10.1002/ 2014GC005652

Manheim, F.T., 1966. A hydraulic squeezer for obtaining interstitial waters from consolidated and unconsolidated sediments. U. S. Geological Survey Professional Paper, 550-C:256-261.

Mehra, O.P., and Jackson, M.L., 1960. Iron oxide removal from soils and clays by a dithionite-citrate system buffered with sodium bicarbonate. Clays and Clay Minerals, 7:317-327. http://dx.doi.org/10.1346/

CCMN.1958.0070122
Muratli, J.M., McManus, J., Mix, A., and Chase, Z., 2012. Dissolution of fluoride complexes following microwaveassisted hydrofluoric acid digestion of marine sediments. Talanta, 89:195-200. http://dx.doi.org/ 10.1016/j.talanta.2011.11.081

Muratli, J.M., Megowan, M.R., and McManus, M., 2015. Data report: sediment major element, minor element, and reactive iron and manganese data from the Okinawa Trough: IODP Expedition 331 Sites C0014 and C0017. In Takai, K., Mottl, M.J., Nielsen, S.H., and the Expedition 331 Scientists, Proceedings of the Integrated Ocean Drilling Program, 331: Tokyo (Integrated Ocean Drilling Program Management International, Inc.). http://dx.doi.org/10.2204/iodp.proc.331.202.2015

Palmer, M.R., Hatter, S.J., Gernon, T.M., Taylor, R.N., Cassidy, M., Johnson, P., Le Friant, A., and Ishiquka, O., 2016. Discovery of a large 2.4 Ma Plinian eruption of Basse-Terre, Guadeloupe, from the marine sediment record. Geology, 44(2):123-126. http://dx.doi.org/ 10.1130/G37193.1

Roy, M., McManus, J., Goñi, M.A., Chase, Z., Borgeld, J.C., Wheatcroft, R.A., Muratli, J.M., Megowan, M.R., and Mix, A., 2013. Reactive iron and manganese distributions in seabed sediments near small mountainous rivers off Oregon and California (USA). Continental Shelf Research, 54:67-69. http://dx.doi.org/10.1016/ j.csr.2012.12.012

Ziebis, W., McManus, J., Ferdelman, T., Schmidt-Schierhorn, F., Bach, W., Muratli, J., Edwards, K.J., and Villinger, H., 2012. Interstitial fluid chemistry of sediments underlying the North Atlantic Gyre and the influence of subsurface fluid flow. Earth and Planetary Science Letters, 323-324:79-91. http://dx.doi.org/10.1016/ j.eps1.2012.01.018

Initial receipt: 6 March 2016

Acceptance: 15 August 2016

Publication: 26 October 2016 MS 340-207 
Figure F1. Map of study area, Expedition 340. Data from sites presented in this report are indicated by solid circles.

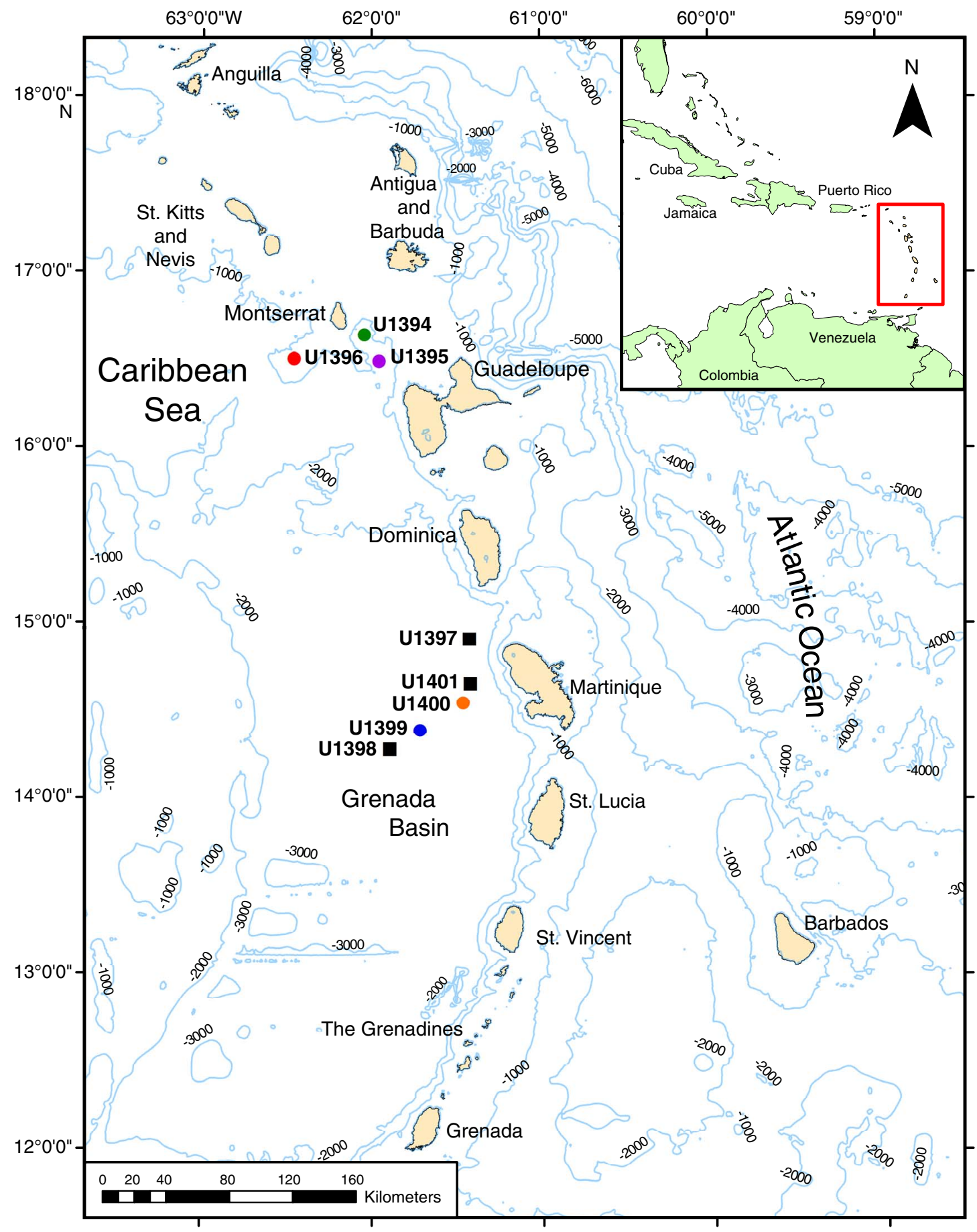


Figure F2. Dissolved pore fluid concentrations as a function of sediment depth, Hole U1394B. A. Ca and Mg. B. S and Mn. C. B and Si. D. Li and Sr. E. Cs and Rb. Dashed lines = bottom water concentrations.
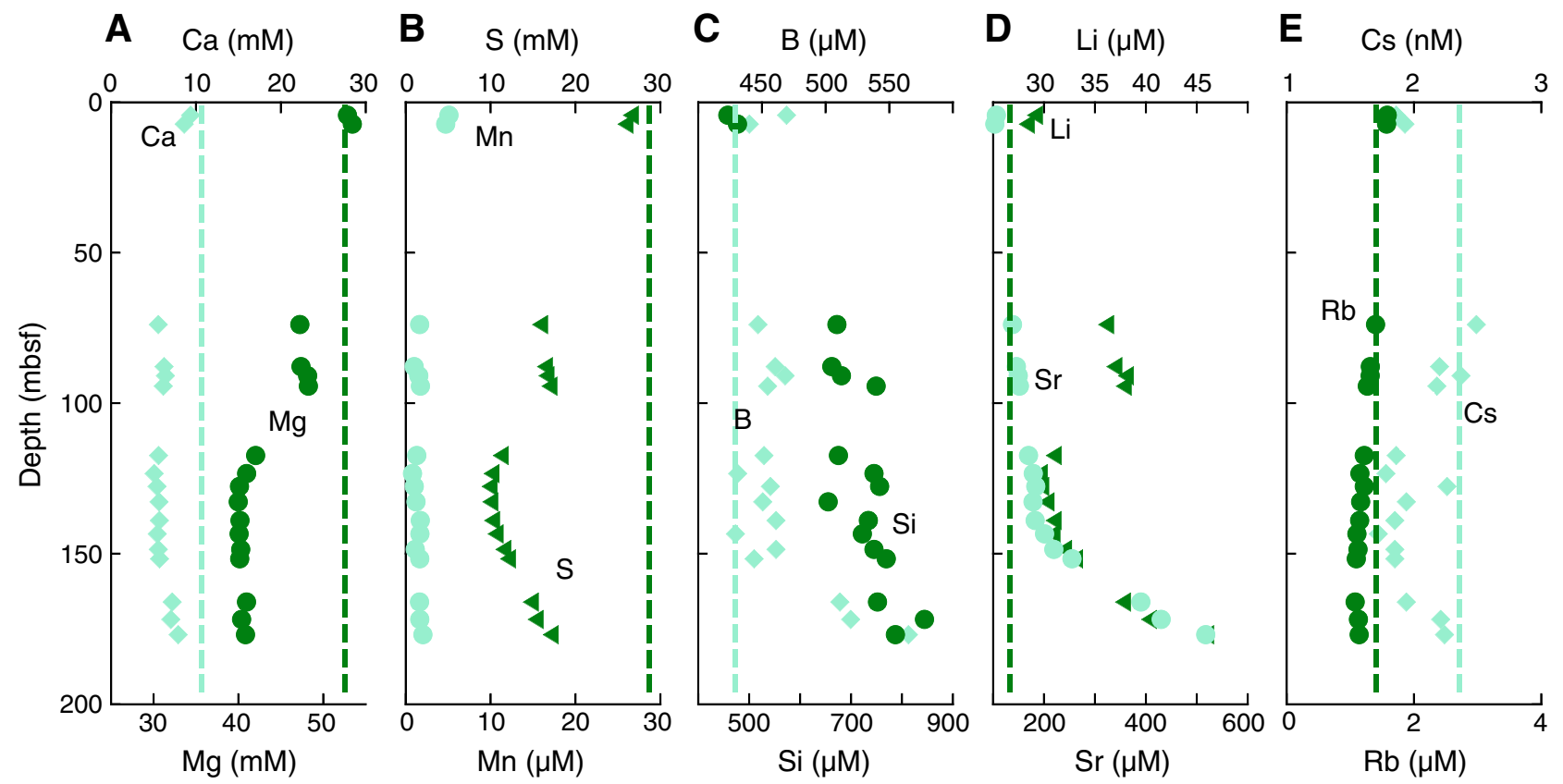

Figure F3. Dissolved pore fluid concentrations as a function of sediment depth, Hole U1395B. A. Ca and Mg. B. S and Mn. C. B and Si. D. Li and Sr. E. Cs and Rb. Dashed lines = bottom water concentrations.
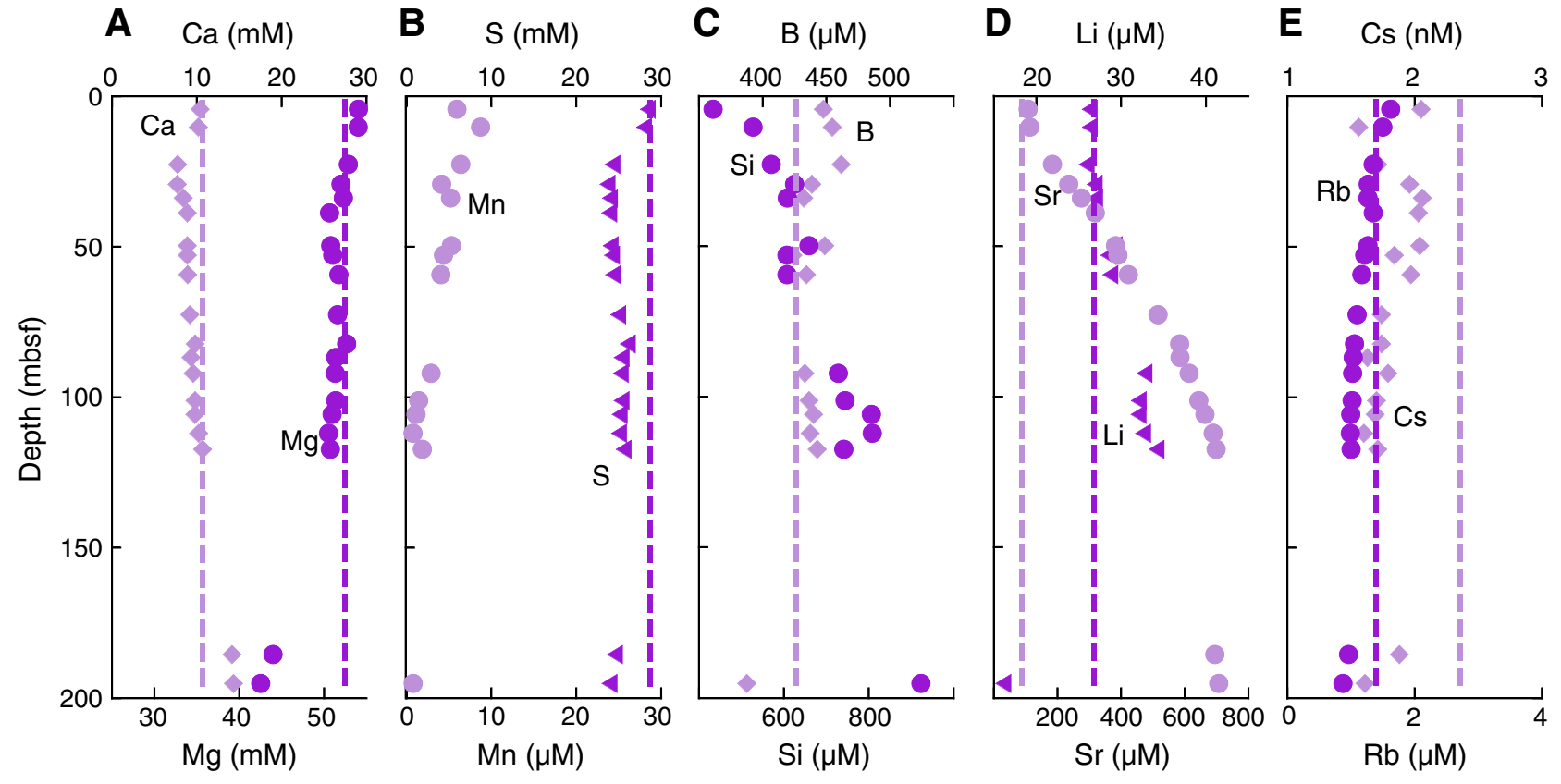
Figure F4. Dissolved pore fluid concentrations as a function of sediment depth, Hole U1396C. A. Ca and Mg. B. S and Mn. C. B and Si. D. Li and Sr. E. Cs and Rb. Dashed lines = bottom water concentrations.

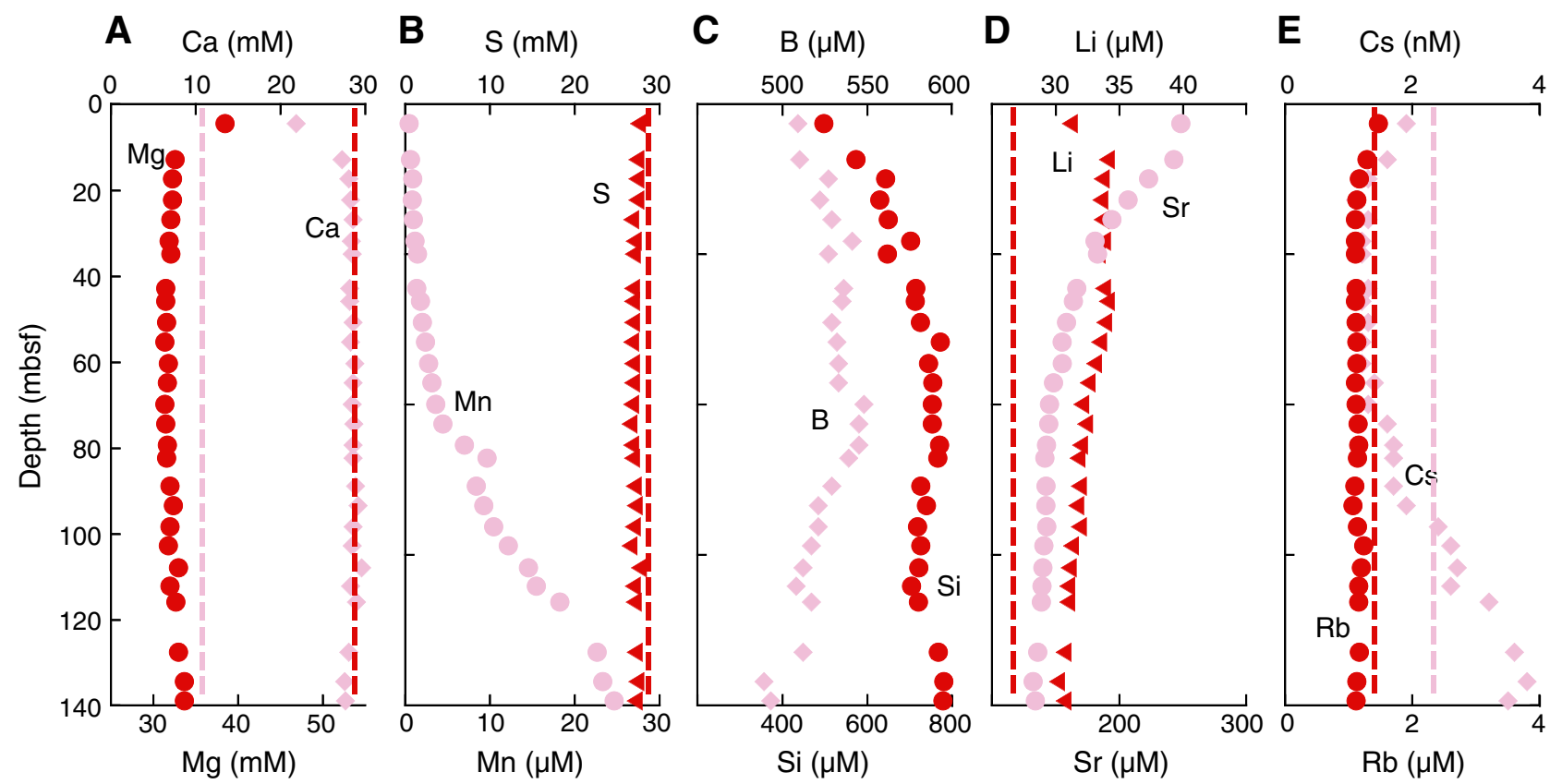

Figure F5. Dissolved pore fluid concentrations as a function of sediment depth, Hole U1399B. A. Ca and Mg. B. S and Mn. C. B and Si. D. Li and Sr. E. Cs and Rb. Dashed lines = bottom water concentrations.

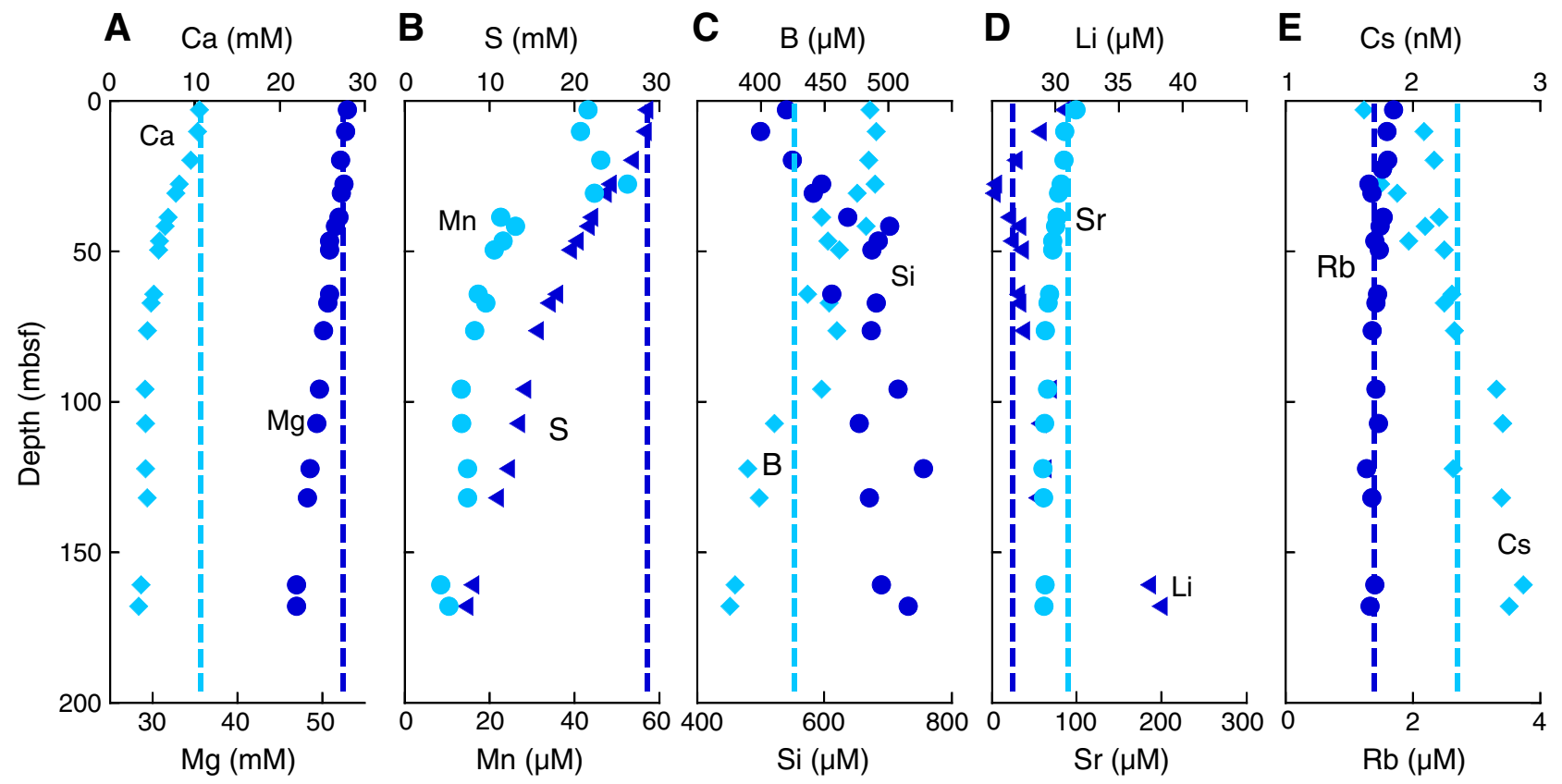


Figure F6. Dissolved pore fluid concentrations as a function of sediment depth, Holes U1400B and U1400C. A. Ca and Mg. B. S and Mn. C. B and Si. D. Li and Sr. E. Cs and Rb. Dashed lines = bottom water concentrations.

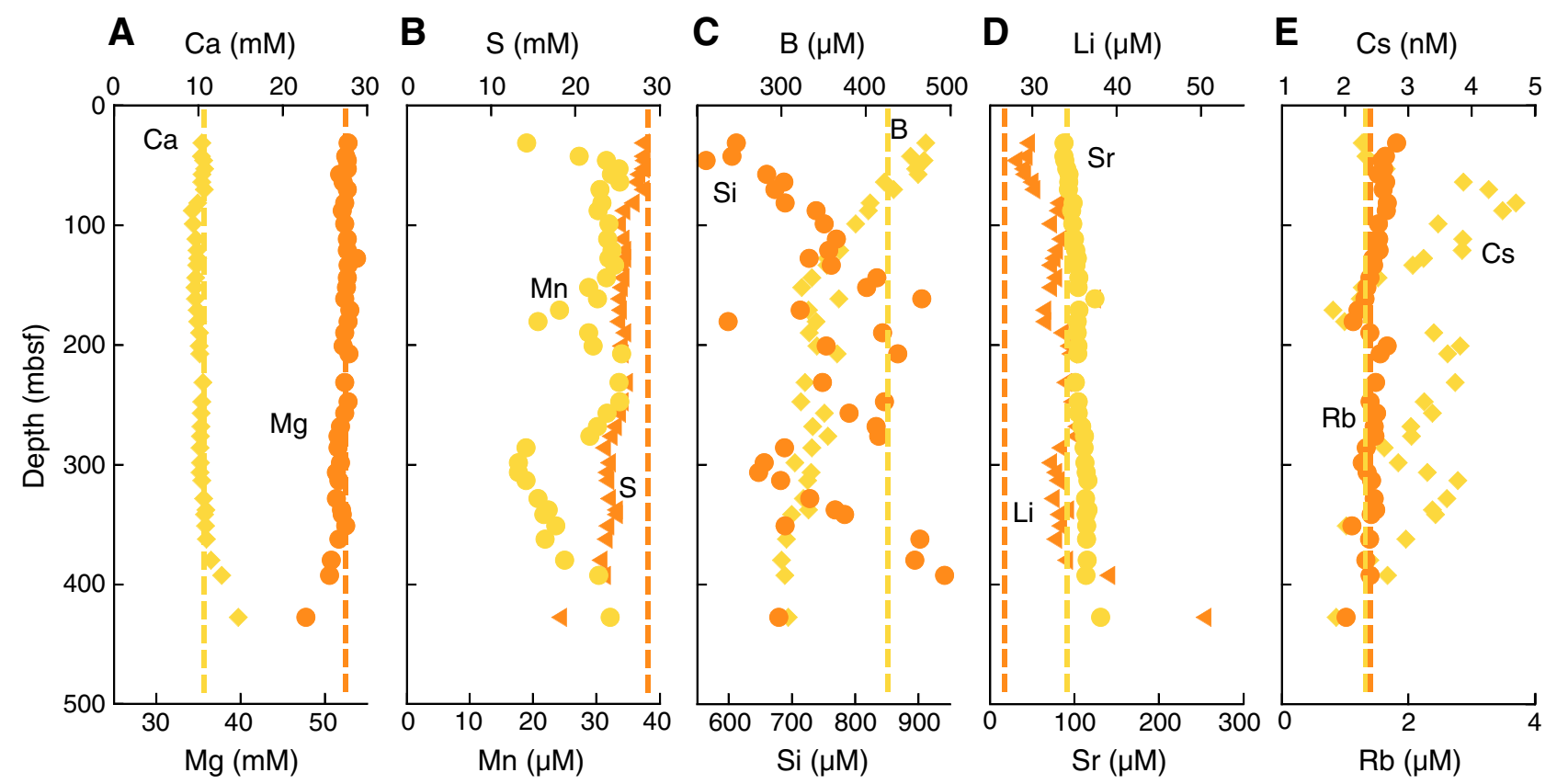

Figure F7. Solid-phase (A) $\mathrm{Fe}_{\mathrm{R}},(\mathbf{B}) \mathrm{Mn}_{\mathrm{R}},(\mathbf{C})$ organic carbon (OC), and (D) $\mathrm{CaCO}_{3}$ vs. sediment depth, Hole U1394A.

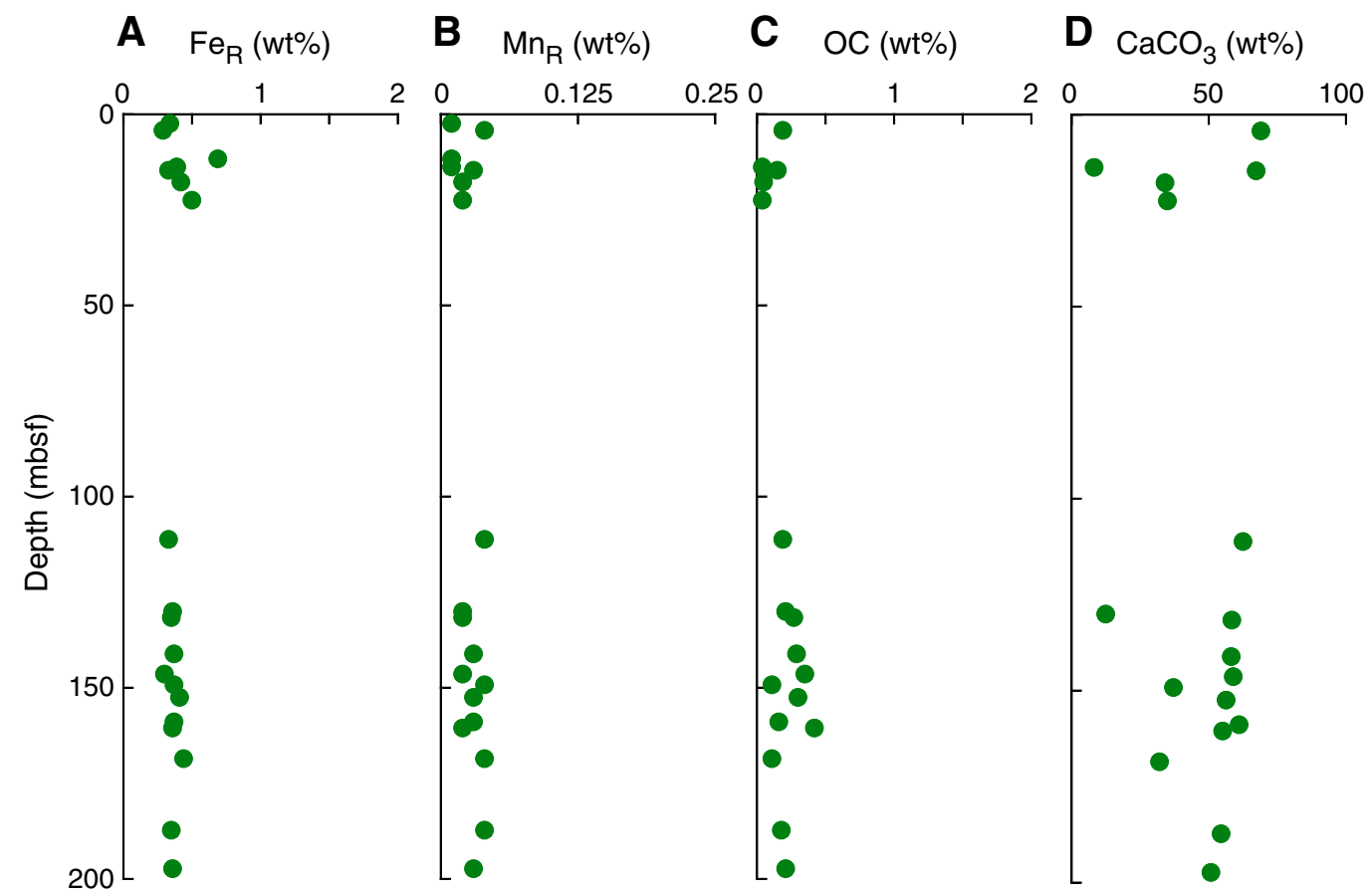


Figure F8. Solid-phase (A) $\mathrm{Fe}_{\mathrm{R}}$, (B) $\mathrm{Mn}_{\mathrm{R}}$, (C) organic carbon (OC), and (D) $\mathrm{CaCO}_{3}$ vs. sediment depth, Hole U1395A.

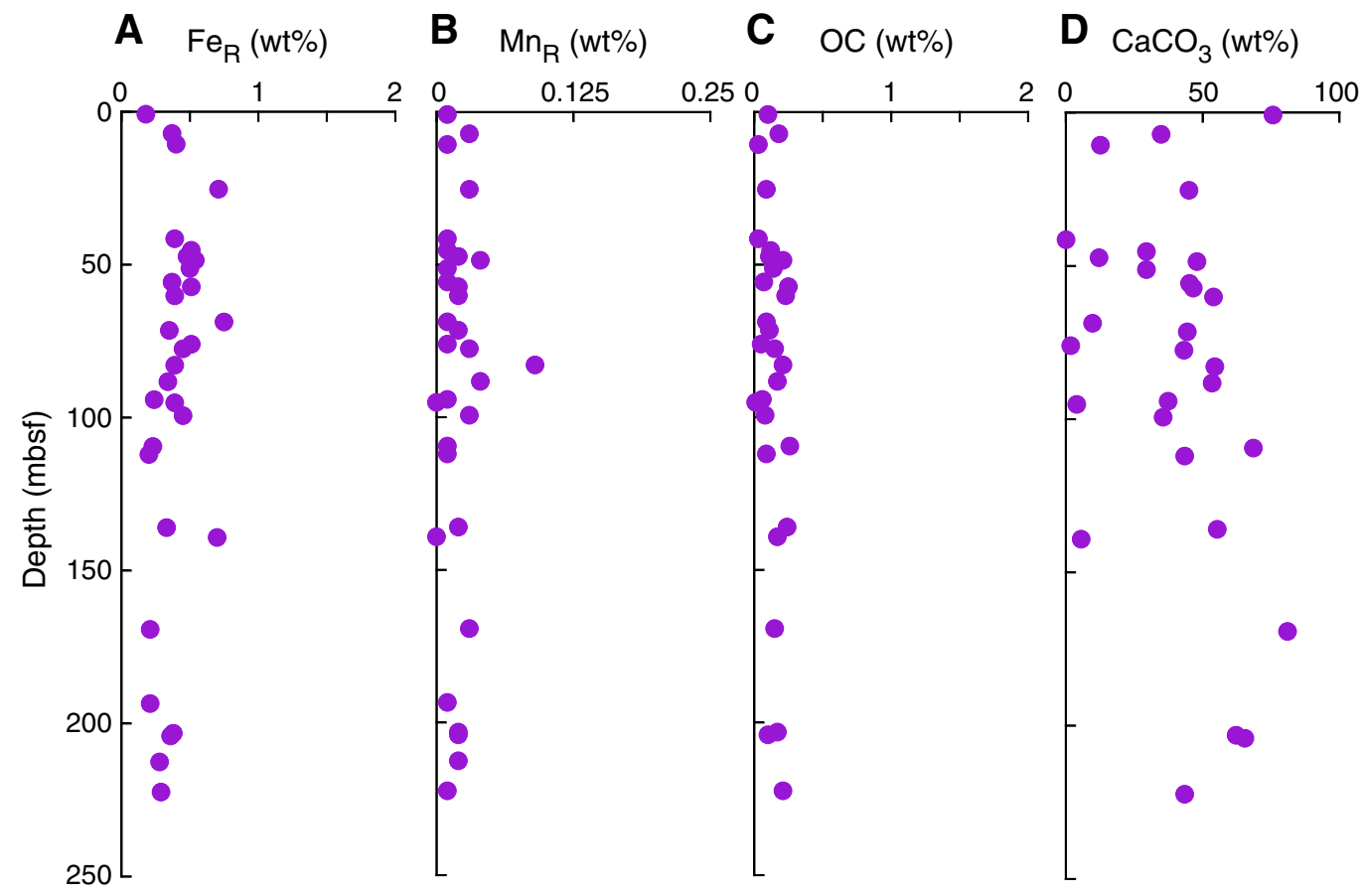

Figure F9. Solid-phase (A) $\mathrm{Fe}_{\mathrm{R}},(\mathbf{B}) \mathrm{Mn}_{\mathrm{R}}$, (C) organic carbon (OC), and (D) $\mathrm{CaCO}_{3}$ vs. sediment depth, Hole U1396A.

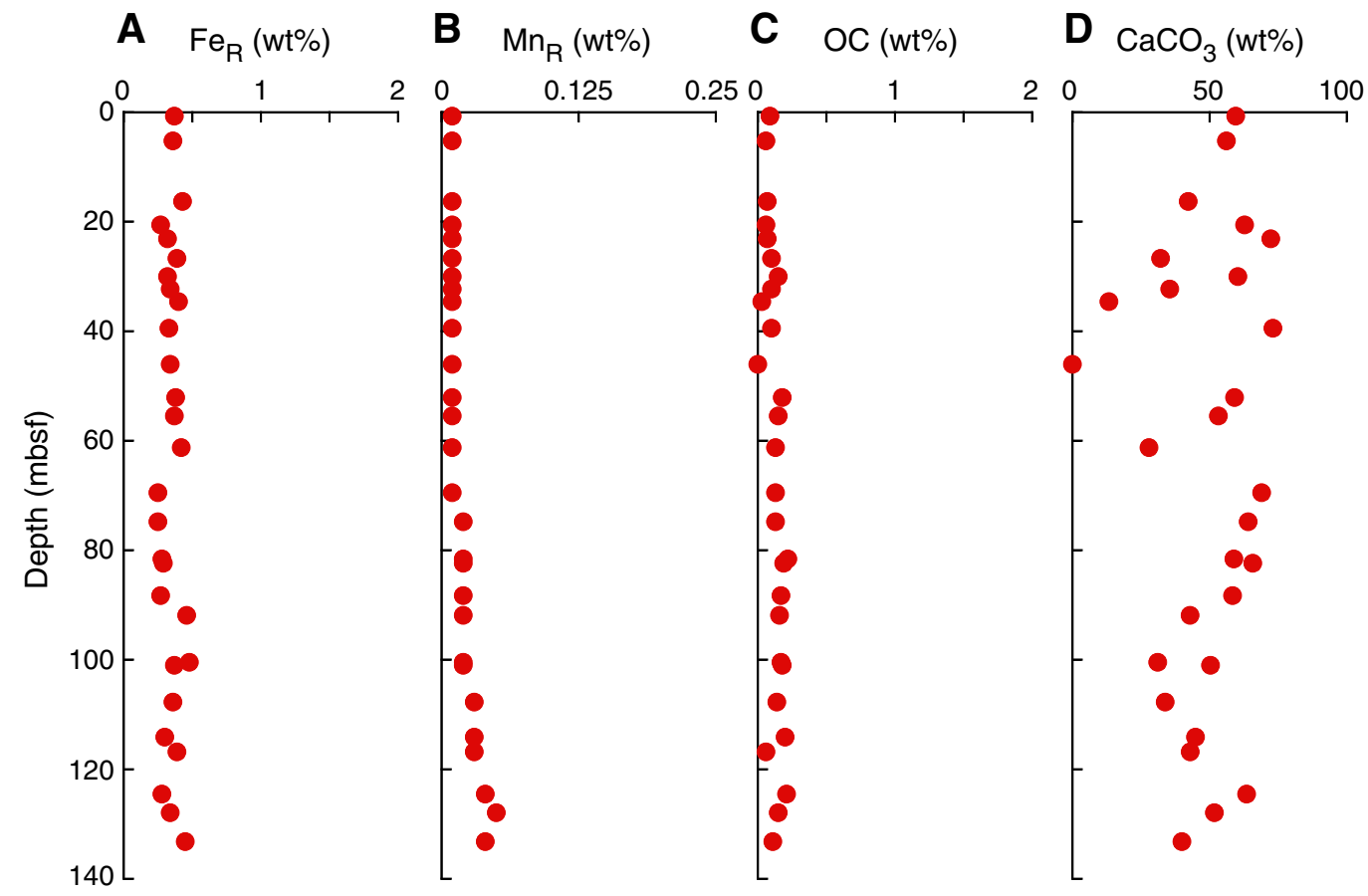


Figure F10. Solid-phase (A) $\mathrm{Fe}_{\mathrm{R}},(\mathbf{B}) \mathrm{Mn}_{\mathrm{R}}$, (C) organic carbon (OC), and (D) $\mathrm{CaCO}_{3}$ vs. sediment depth, Hole U1399A.

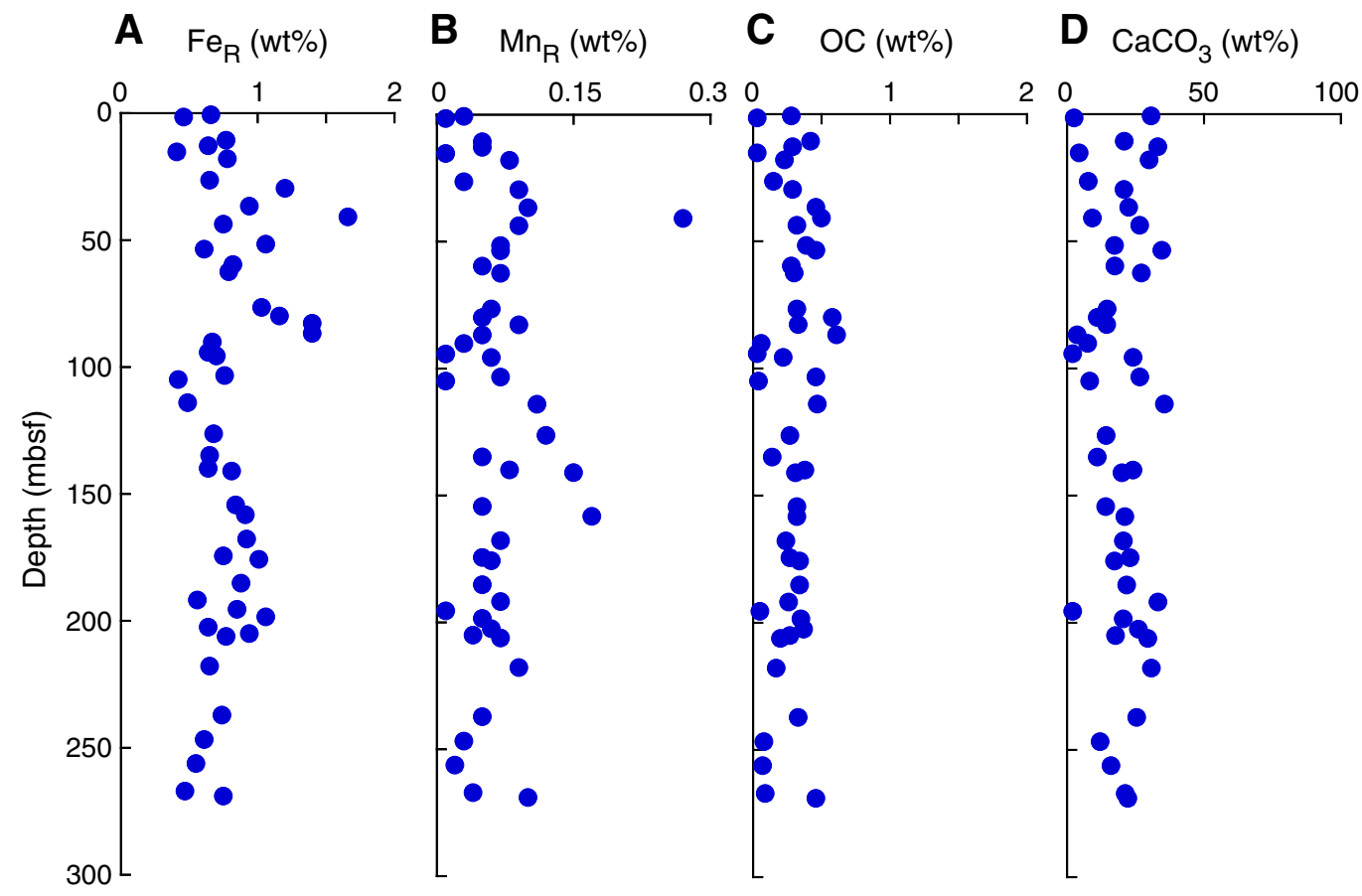

Figure F11. Solid-phase (A) $\mathrm{Fe}_{\mathrm{R}},(\mathbf{B}) \mathrm{Mn}_{\mathrm{R}},(\mathbf{C})$ organic carbon (OC), and (D) $\mathrm{CaCO}_{3}$ vs. sediment depth, Holes U1400B and U1400C.

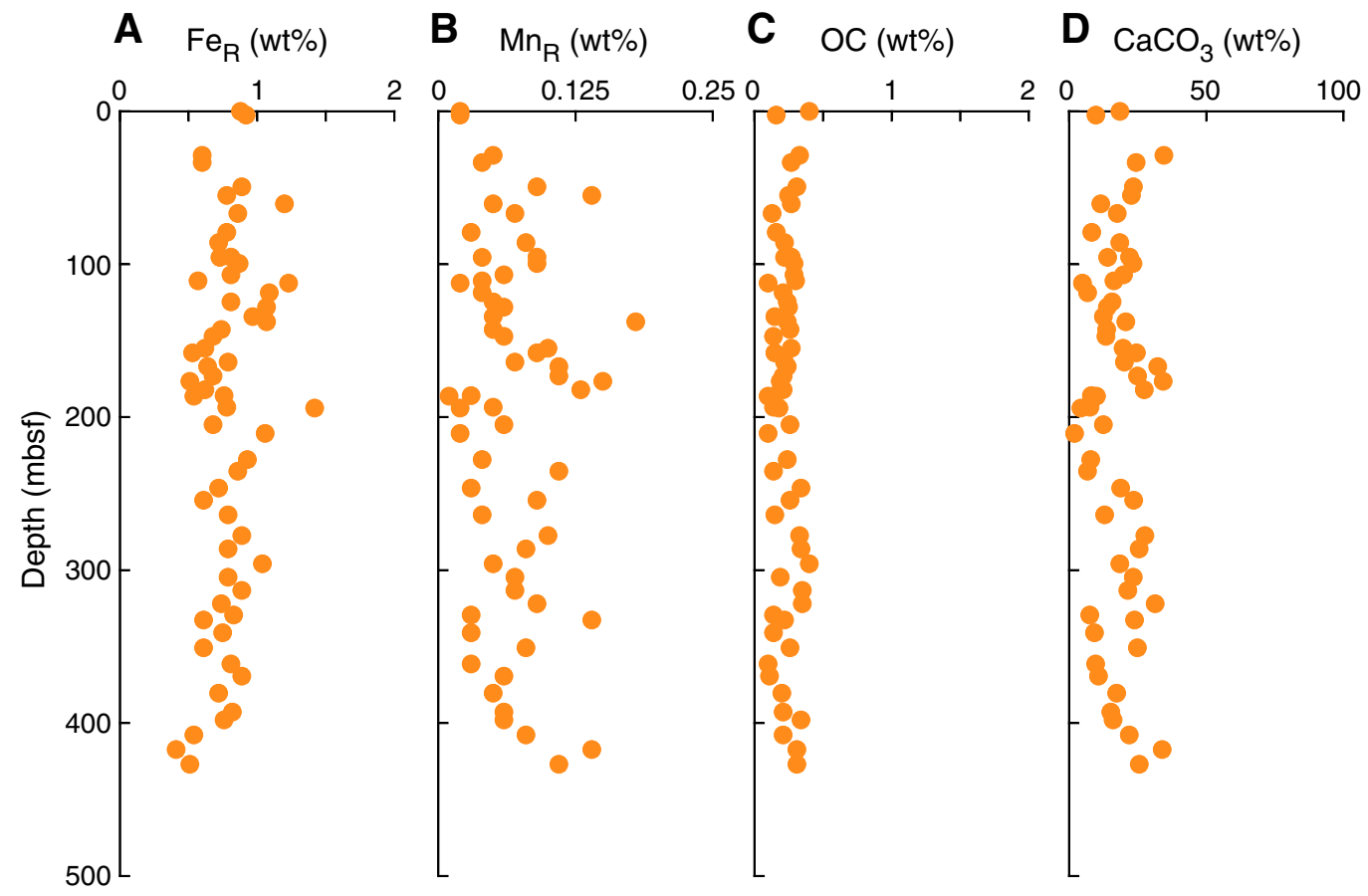


Figure F12. Solid-phase (A) $\mathrm{Fe}_{\mathrm{R}},(\mathbf{B}) \mathrm{Mn}_{\mathrm{R}},(\mathbf{C})$ organic carbon (OC), and (D) $\mathrm{CaCO}_{3}$ vs. sediment depth, Section 340-U1399B-3H-3. Core photo retrieved from on-board data archive (http://web.iodp.tamu.edu/LORE/).

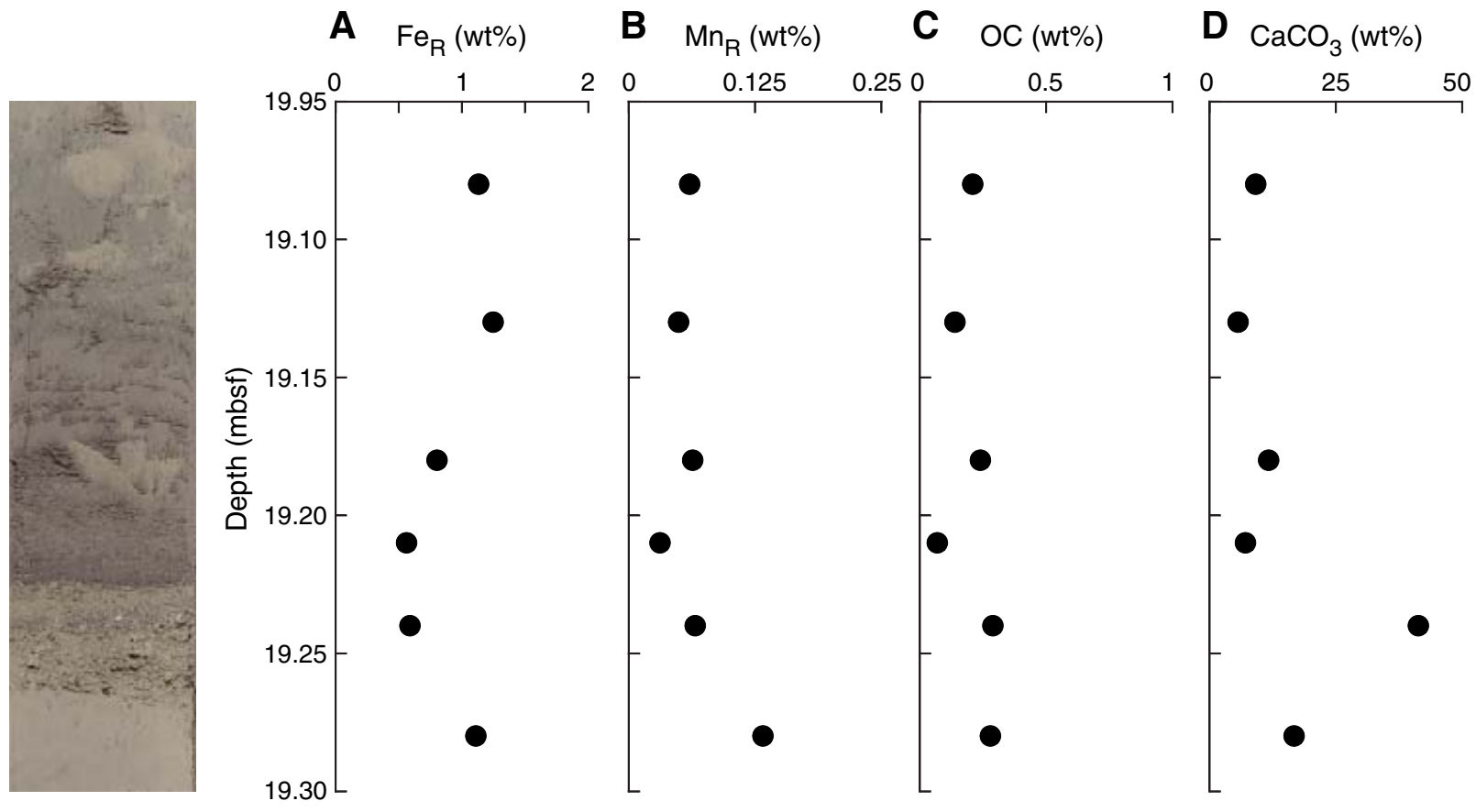

Figure F13. Solid-phase (A) $\mathrm{Fe}_{\mathrm{R}},(\mathbf{B}) \mathrm{Mn}_{\mathrm{R}},(\mathrm{C})$ organic carbon (OC), and (D) $\mathrm{CaCO}_{3}$ vs. sediment depth, Section 340-U1399B-7H-3. Core photo retrieved from onboard data archive (http://web.iodp.tamu.edu/LORE/).

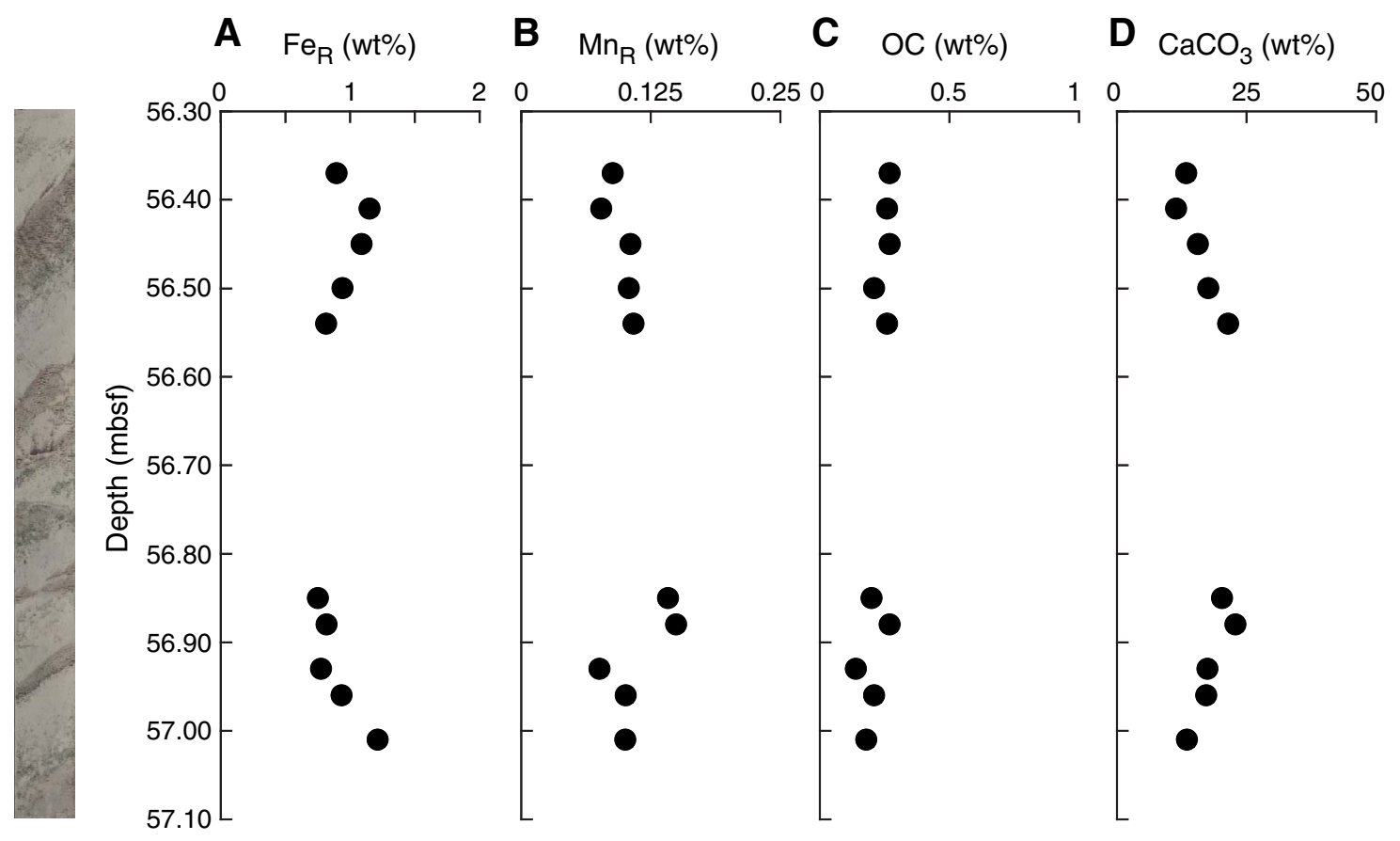


Figure F14. Solid-phase (A) $\mathrm{Fe}_{\mathrm{R}},(\mathbf{B}) \mathrm{Mn}_{\mathrm{R}},(\mathrm{C})$ organic carbon (OC), and (D) $\mathrm{CaCO}_{3}$ vs. sediment depth, Section 340-U1399B-11H-2. Core photo retrieved from onboard data archive (http://web.iodp.tamu.edu/LORE/).

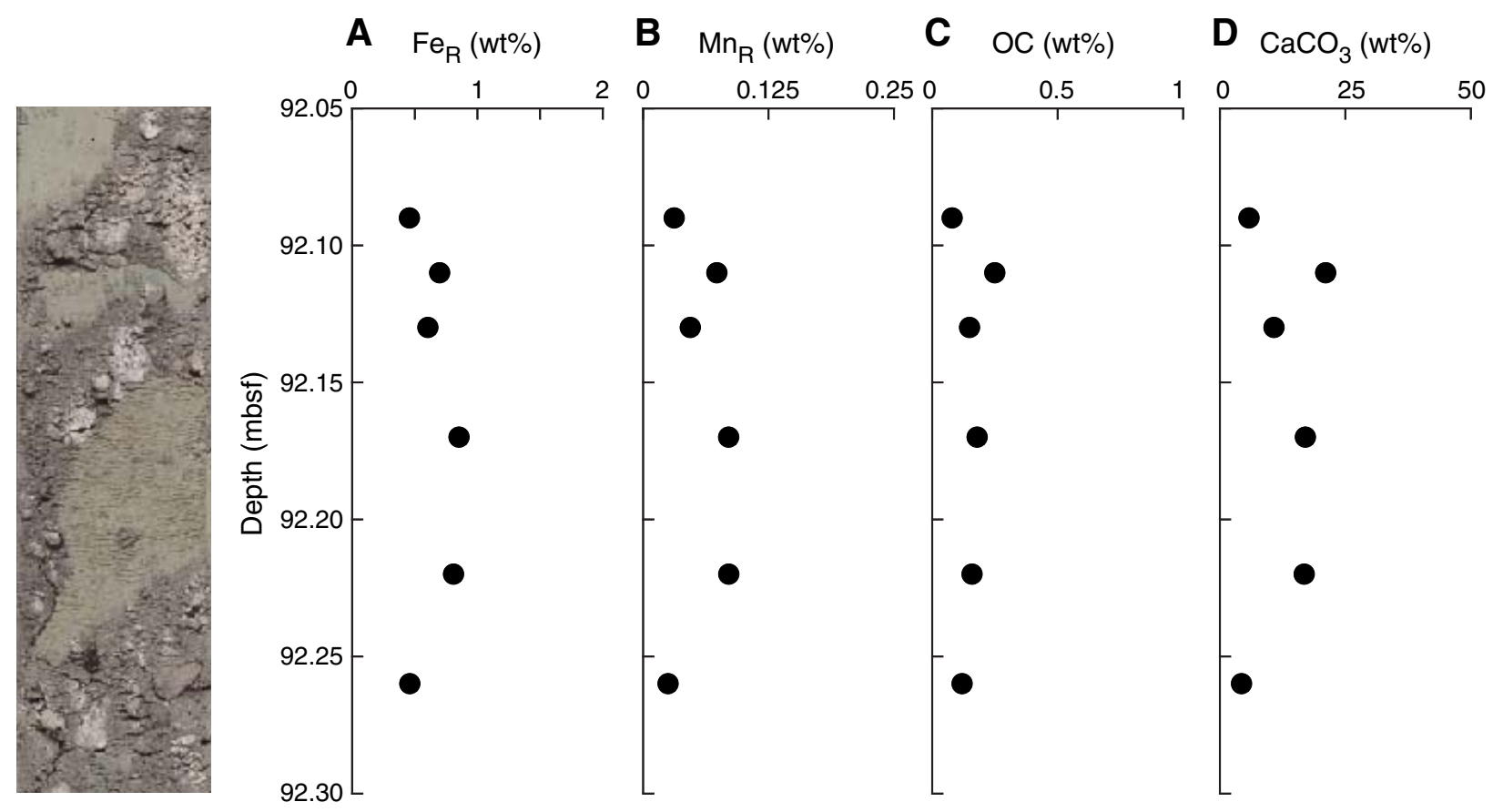

Figure F15. Solid-phase (A) $\mathrm{Fe}_{\mathrm{R}},(\mathbf{B}) \mathrm{Mn}_{\mathrm{R}},(\mathbf{C})$ organic carbon (OC), and (D) $\mathrm{CaCO}_{3}$ vs. sediment depth, Section 340-U1399B-16H-2. Core photo retrieved from onboard data archive (http://web.iodp.tamu.edu/LORE/).

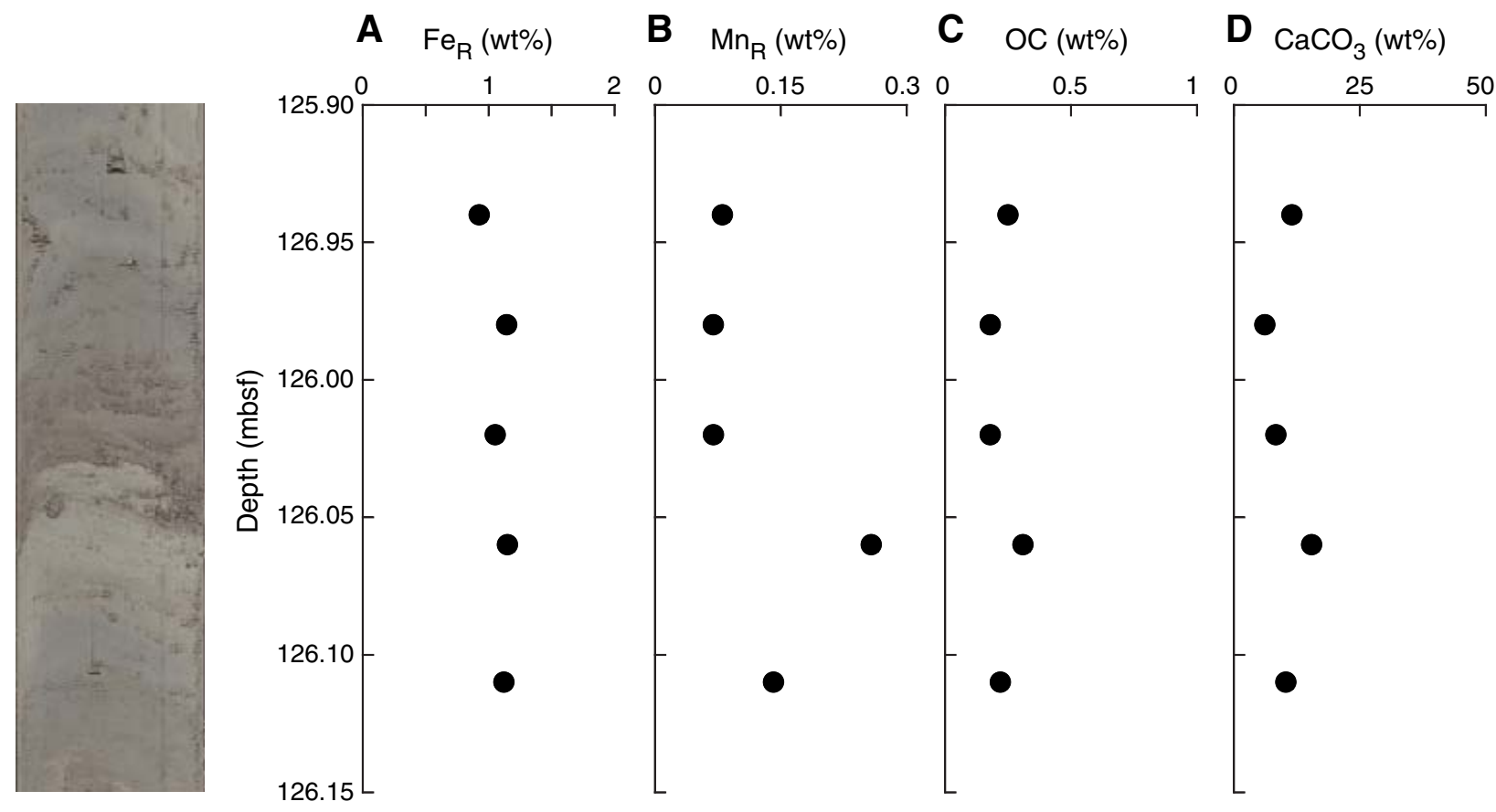


Figure F16. Solid-phase (A) $\mathrm{Fe}_{\mathrm{R}},(\mathbf{B}) \mathrm{Mn}_{\mathrm{R}},(\mathrm{C})$ organic carbon (OC), and (D) $\mathrm{CaCO}_{3}$ vs. sediment depth, Section 340-U1399B-19H-2. Core photo retrieved from onboard data archive (http://web.iodp.tamu.edu/LORE/).

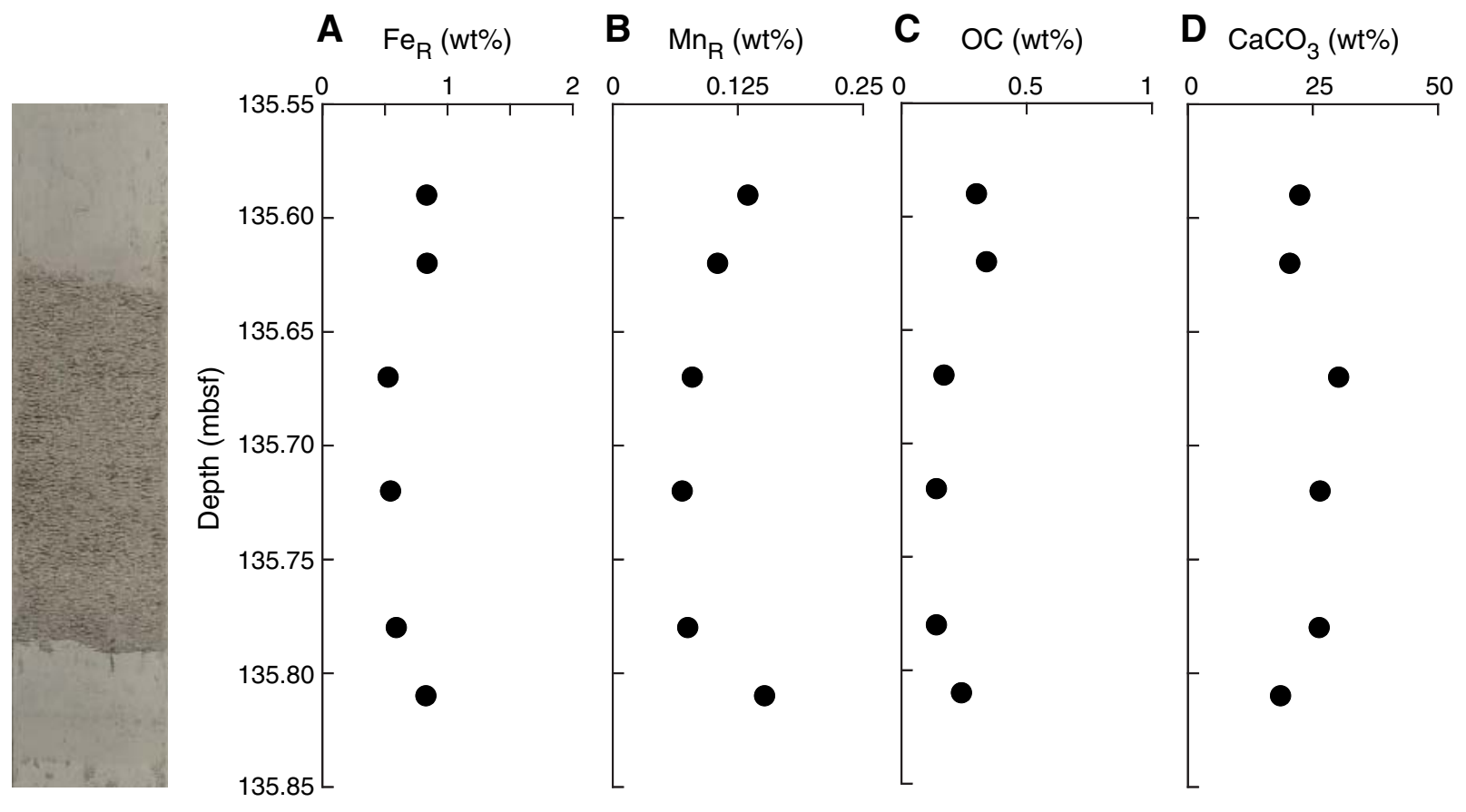

Figure F17. Solid-phase (A) $\mathrm{Fe}_{\mathrm{R}},(\mathbf{B}) \mathrm{Mn}_{\mathrm{R}},(\mathbf{C})$ organic carbon (OC), and (D) $\mathrm{CaCO}_{3}$ vs. sediment depth, Section 340-U1399B-25H-2. Core photo retrieved from onboard data archive (http://web.iodp.tamu.edu/LORE/).

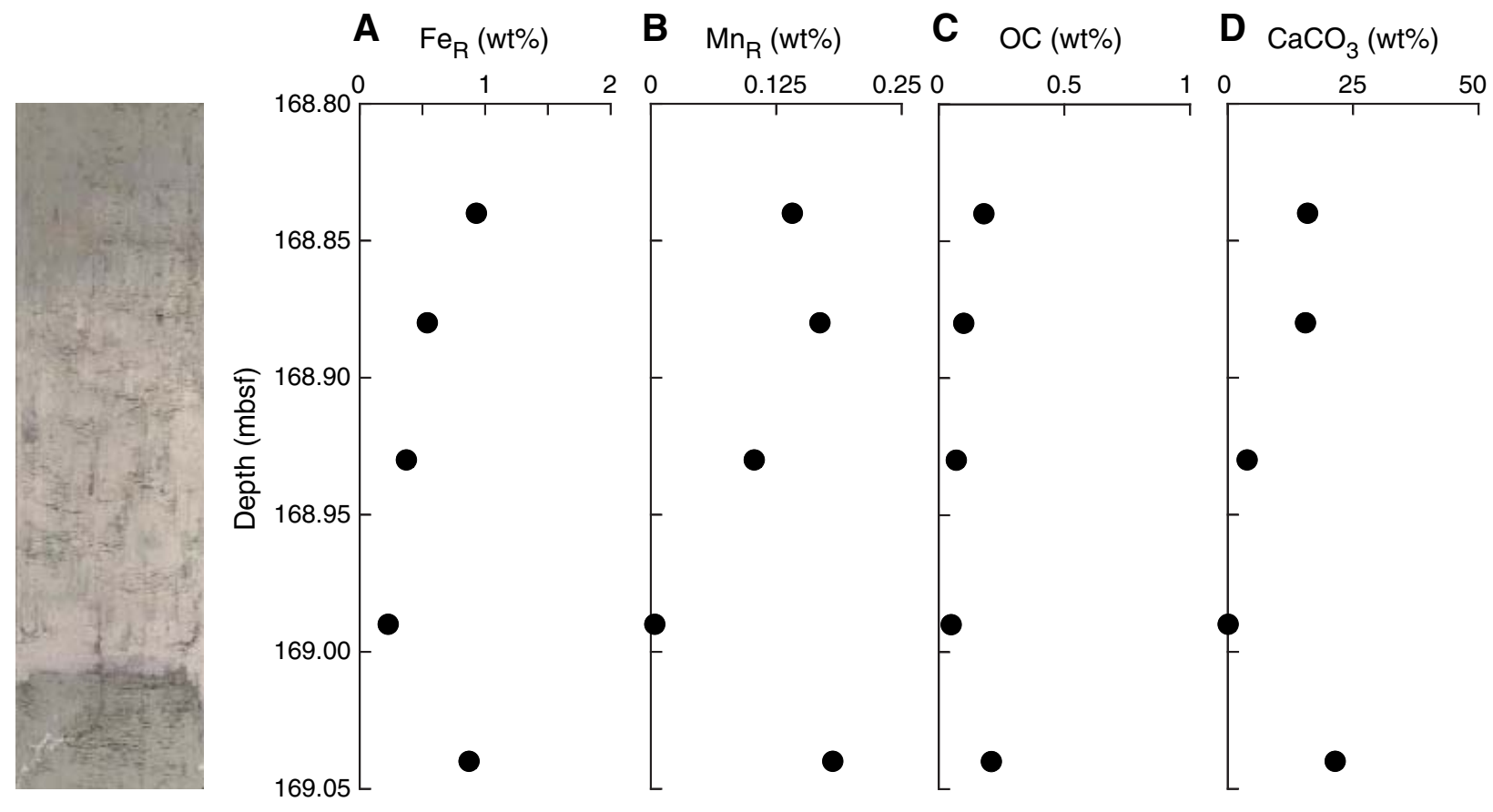


Figure F18. Solid-phase (A) $\mathrm{Fe}_{\mathrm{R}},(\mathbf{B}) \mathrm{Mn}_{\mathrm{R}},(\mathbf{C})$ organic carbon (OC), and (D) $\mathrm{CaCO}_{3}$ vs. sediment depth, Section 340-U1400B-16H-2. Core photo retrieved from onboard data archive (http://web.iodp.tamu.edu/LORE/).

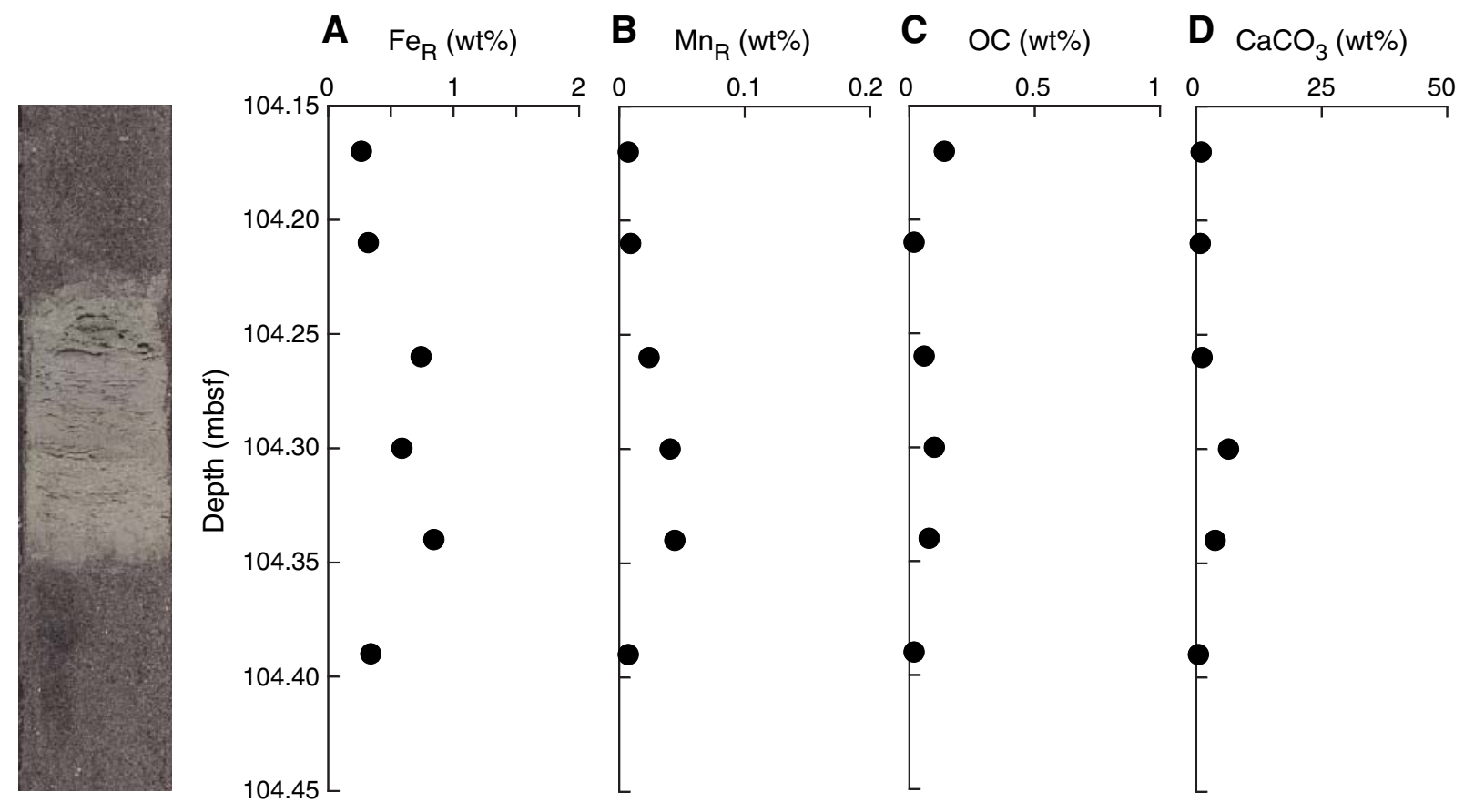

Figure F19. Solid-phase (A) $\mathrm{Fe}_{\mathrm{R}},(\mathbf{B}) \mathrm{Mn}_{\mathrm{R}},(\mathbf{C})$ organic carbon (OC), and (D) $\mathrm{CaCO}_{3}$ vs. sediment depth, Section 340-U1400B-26H-5. Core photo retrieved from onboard data archive (http://web.iodp.tamu.edu/LORE/).

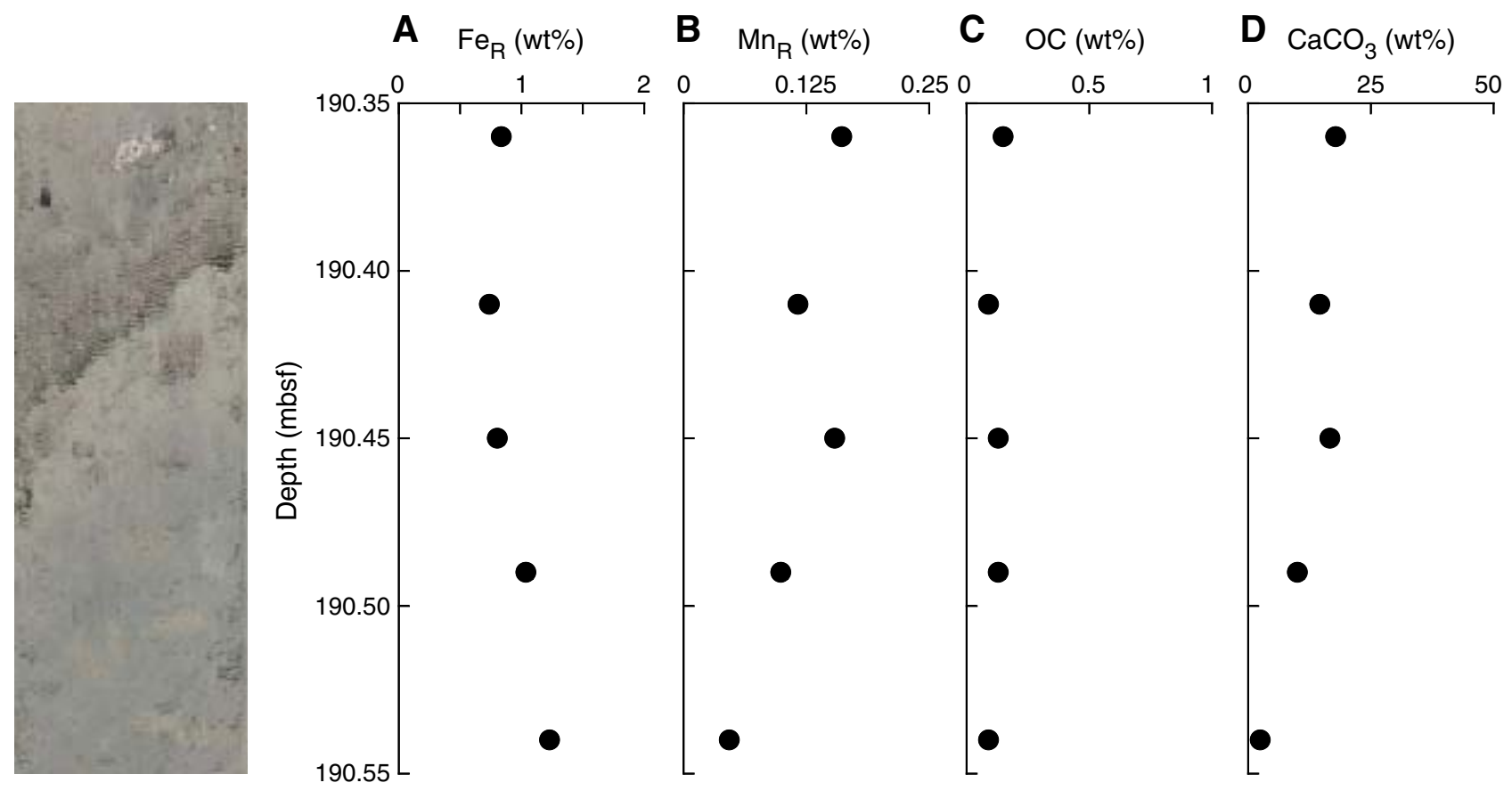


Figure F20. Solid-phase (A) $\mathrm{Fe}_{\mathrm{R}},(\mathbf{B}) \mathrm{Mn}_{\mathrm{R}},(\mathbf{C})$ organic carbon (OC), and (D) $\mathrm{CaCO}_{3}$ vs. sediment depth, Section 340-U1400C-48X-1. Core photo retrieved from onboard data archive (http://web.iodp.tamu.edu/LORE/).

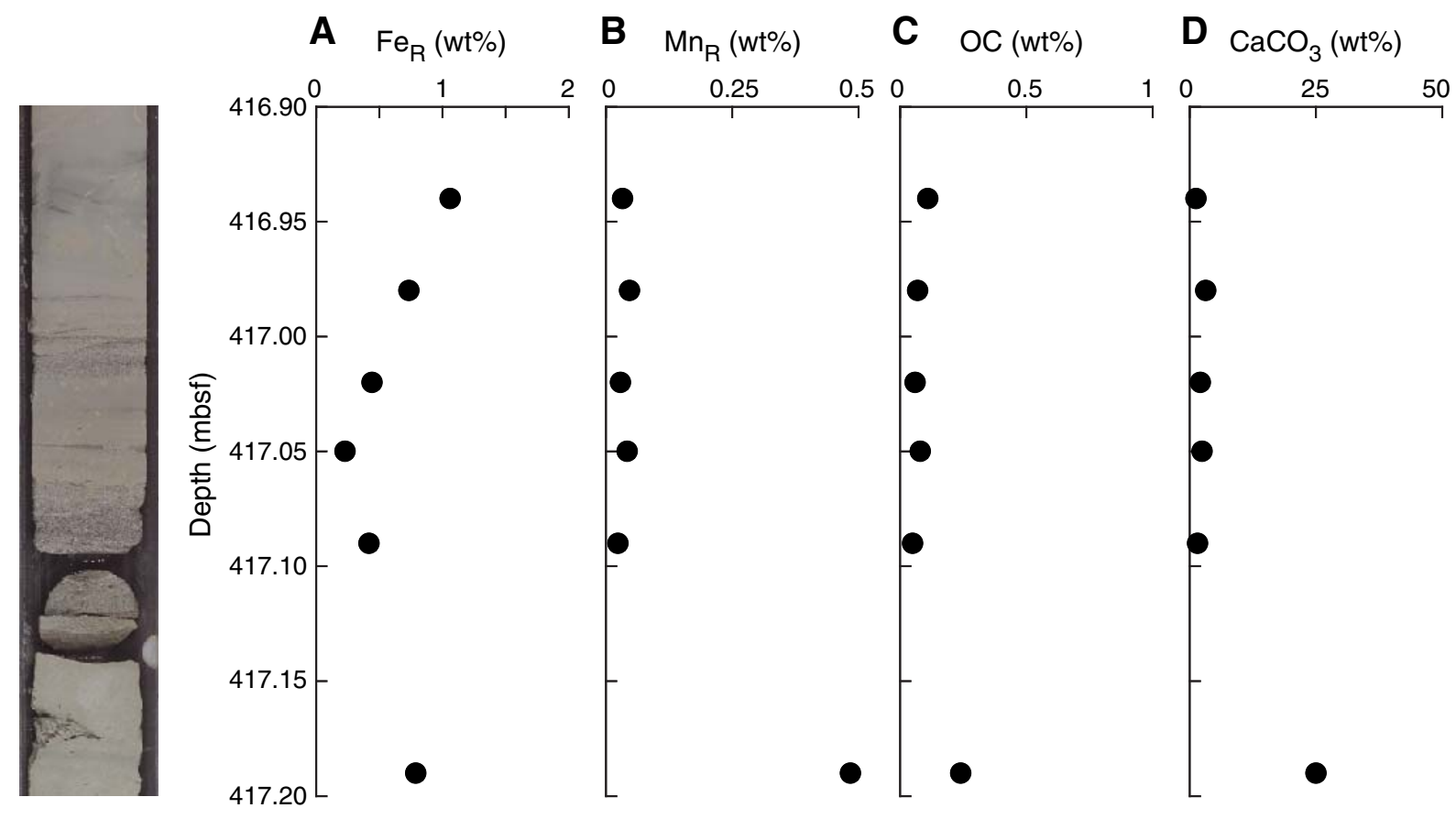


Table T1. Pore fluid composition, Holes U1394B, U1395B, U1396C, U1399B, U1400B, and U1400C. (Continued on next page.)

\begin{tabular}{|c|c|c|c|c|c|c|c|c|c|c|c|c|c|c|c|c|}
\hline \multirow{2}{*}{$\begin{array}{l}\text { Core, } \\
\text { section }\end{array}$} & \multicolumn{2}{|c|}{ Depth CSF-A (m) } & \multirow{2}{*}{$\begin{array}{c}\mathrm{Li} \\
(\mu \mathrm{M})\end{array}$} & \multirow{2}{*}{$\stackrel{ \pm}{(\mu \mathrm{M})}$} & \multirow{2}{*}{$\begin{array}{c}B \\
(\mu \mathrm{M})\end{array}$} & \multirow{2}{*}{$\stackrel{ \pm}{ \pm}(\mu \mathrm{M})$} & \multirow{2}{*}{$\begin{array}{c}\mathrm{Si} \\
(\mu \mathrm{M})\end{array}$} & \pm & $\mathrm{Mn}$ & $I$ & $\mathrm{Sr}$ & \pm & $\mathrm{Rb}$ & \pm & Cs & \pm \\
\hline & Top & Bottom & & & & & & $(\mu \mathrm{M})$ & $(\mu \mathrm{M})$ & $(\mu \mathrm{M})$ & $(\mu \mathrm{M})$ & $(\mu \mathrm{M})$ & $(\mu \mathrm{M})$ & $(\mu \mathrm{M})$ & $(\mathrm{pM})$ & (pM) \\
\hline 340-U139 & & & & & & & & & & & & & & & & \\
\hline $1 \mathrm{H}-3$ & 4.40 & 4.45 & 29.1 & 0.6 & 469 & 3 & 458 & 3 & 5.08 & 0.04 & 106 & 1 & 1.58 & 0.02 & 1.9 & 0.2 \\
\hline $1 \mathrm{H}-5$ & 7.30 & 7.35 & 28.3 & 0.7 & 440 & 5 & 477 & 5 & 4.71 & 0.06 & 103 & 1 & 1.57 & 0.02 & 1.9 & 0.1 \\
\hline $10 \mathrm{H}-1$ & 73.90 & 73.95 & 36.1 & 0.6 & 447 & 1 & 672 & 3 & 1.63 & 0.04 & 138 & 1 & 1.40 & 0.01 & 2.5 & 0.2 \\
\hline $11 \mathrm{H}-4$ & 87.92 & 88.02 & 36.9 & 0.6 & 460 & 3 & 662 & 6 & 0.99 & 0.04 & 146 & 1 & 1.31 & 0.02 & 2.2 & 0.1 \\
\hline $11 \mathrm{H}-6$ & 90.92 & 91.02 & 38.1 & 0.6 & 468 & 2 & 681 & 7 & 1.54 & 0.04 & 149 & 1 & 1.31 & 0.02 & 2.4 & 0.2 \\
\hline $12 \mathrm{H}-2$ & 94.40 & 94.50 & 37.8 & 0.6 & 455 & 2 & 749 & 3 & 1.72 & 0.04 & 152 & 1 & 1.27 & 0.01 & 2.2 & 0.1 \\
\hline $14 \mathrm{H}-5$ & 117.40 & 117.50 & 31.0 & 0.6 & 452 & 4 & 675 & 5 & 1.30 & 0.04 & 170 & 1 & 1.22 & 0.02 & 1.9 & 0.1 \\
\hline $15 \mathrm{H}-3$ & 123.40 & 123.50 & 29.6 & 0.6 & 431 & 3 & 745 & 3 & 0.79 & 0.04 & 179 & 1 & 1.15 & 0.01 & 1.8 & 0.1 \\
\hline $15 \mathrm{H}-6$ & 127.72 & 127.82 & 29.7 & 0.6 & 457 & 2 & 756 & 5 & 1.01 & 0.04 & 184 & 1 & 1.22 & 0.02 & 2.3 & 0.1 \\
\hline $16 \mathrm{H}-3$ & 132.80 & 132.90 & 30.3 & 0.6 & 451 & 1 & 655 & 4 & 1.20 & 0.04 & 178 & 1 & 1.16 & 0.02 & 1.9 & 0.1 \\
\hline $17 \mathrm{H}-3$ & 139.00 & 139.10 & 30.9 & 0.6 & 461 & 4 & 734 & 5 & 1.70 & 0.06 & 183 & 1 & 1.15 & 0.01 & 1.9 & 0.2 \\
\hline $17 \mathrm{H}-6$ & 143.46 & 143.56 & 30.8 & 0.3 & 429 & 1 & 722 & 3 & 1.66 & 0.07 & 201 & 5 & 1.11 & 0.02 & 1.7 & 0.1 \\
\hline $18 \mathrm{H}-3$ & 148.60 & 148.70 & 32.0 & 0.6 & 461 & 2 & 745 & 5 & 1.12 & 0.04 & 219 & 5 & 1.12 & 0.01 & 1.8 & 0.1 \\
\hline $18 \mathrm{H}-6$ & 151.72 & 151.82 & 33.1 & 0.6 & 444 & 6 & 769 & 5 & 1.65 & 0.04 & 255 & 5 & 1.09 & 0.01 & 1.8 & 0.1 \\
\hline $20 \mathrm{H}-2$ & 166.10 & 166.20 & 37.8 & 0.6 & 511 & 3 & 752 & 3 & 1.62 & 0.05 & 390 & 5 & 1.08 & 0.01 & 1.9 & 0.2 \\
\hline $20 \mathrm{H}-6$ & 171.87 & 171.97 & 40.3 & 0.3 & 520 & 0 & 844 & 2 & 1.64 & 0.05 & 430 & 5 & 1.13 & 0.01 & 2.2 & 0.1 \\
\hline $21 \mathrm{H}-3$ & 176.93 & 177.03 & 45.9 & 0.6 & 565 & 2 & 787 & 5 & 2.02 & 0.04 & 517 & 5 & 1.14 & 0.02 & 2.2 & 0.1 \\
\hline 340-U139 & & & & & & & & & & & & & & & & \\
\hline $1 \mathrm{H}-3$ & 4.40 & 4.50 & 26.3 & 0.6 & 448 & 1 & 433 & 3 & 5.93 & 0.04 & 108 & 1 & 1.62 & 0.03 & 2.0 & 0.2 \\
\hline $2 \mathrm{H}-3$ & 10.30 & 10.40 & 26.3 & 0.6 & 455 & 3 & 527 & 3 & 8.72 & 0.08 & 113 & 1 & 1.50 & 0.01 & 1.6 & 0.1 \\
\hline $3 \mathrm{H}-5$ & 22.70 & 22.80 & 25.9 & 0.6 & 462 & 1 & 570 & 3 & 6.39 & 0.05 & 184 & 1 & 1.35 & 0.01 & 1.7 & 0.2 \\
\hline $4 \mathrm{H}-3$ & 29.32 & 29.42 & 26.9 & 0.6 & 439 & 2 & 625 & 4 & 4.13 & 0.04 & 235 & 5 & 1.27 & 0.02 & 2.0 & 0.1 \\
\hline $4 \mathrm{H}-6$ & 33.88 & 33.98 & 26.9 & 0.6 & 432 & 3 & 608 & 3 & 5.18 & 0.05 & 275 & 5 & 1.26 & 0.02 & 2.1 & 0.1 \\
\hline $5 \mathrm{H}-3$ & 38.80 & 38.90 & & & & & & & & & 318 & 5 & 1.35 & 0.02 & 2.0 & 0.1 \\
\hline $6 \mathrm{H}-4$ & 49.72 & 49.82 & 29.2 & 0.6 & 449 & 2 & 659 & 3 & 5.28 & 0.04 & 382 & 5 & 1.27 & 0.02 & 2.0 & 0.2 \\
\hline $6 \mathrm{H}-6$ & 52.85 & 52.95 & 28.5 & 0.6 & 424 & 3 & 607 & 3 & 4.35 & 0.05 & 390 & 6 & 1.21 & 0.01 & 1.8 & 0.1 \\
\hline $7 \mathrm{H}-4$ & 59.31 & 59.41 & 28.7 & 0.6 & 434 & 4 & 607 & 3 & 4.04 & 0.04 & 422 & 6 & 1.17 & 0.02 & 2.0 & 0.2 \\
\hline $9 \mathrm{H}-1$ & 72.60 & 72.70 & & & & & & & & & 516 & 5 & 1.10 & 0.01 & 1.7 & 0.1 \\
\hline $10 \mathrm{H}-2$ & 82.30 & 82.40 & & & & & & & & & 584 & 5 & 1.05 & 0.02 & 1.7 & 0.1 \\
\hline $10 \mathrm{H}-5$ & 86.81 & 86.91 & & & & & & & & & 585 & 6 & 1.03 & 0.02 & 1.6 & 0.2 \\
\hline $11 \mathrm{H}-3$ & 92.10 & 92.20 & 32.8 & 0.6 & 433 & 2 & 728 & 5 & 2.88 & 0.04 & 614 & 6 & 1.03 & 0.01 & 1.8 & 0.1 \\
\hline $12 \mathrm{H}-3$ & 101.15 & 101.25 & 32.1 & 0.6 & 436 & 1 & 744 & 4 & 1.43 & 0.04 & 644 & 6 & 1.02 & 0.01 & 1.7 & 0.1 \\
\hline $12 \mathrm{H}-6$ & 105.70 & 105.80 & 32.0 & 0.6 & 440 & 3 & 806 & 3 & 1.11 & 0.04 & 663 & 5 & 0.99 & 0.02 & 1.7 & 0.1 \\
\hline $13 \mathrm{H}-4$ & 112.02 & 112.12 & 32.6 & 0.6 & 437 & 4 & 808 & 3 & 0.77 & 0.04 & 689 & 5 & 0.99 & 0.01 & 1.6 & 0.2 \\
\hline $14 \mathrm{H}-3$ & 117.30 & 117.40 & 34.2 & 0.6 & 443 & 3 & 741 & 4 & 1.85 & 0.04 & 698 & 7 & 1.00 & 0.02 & 1.7 & 0.2 \\
\hline $24 X-1$ & 185.50 & 185.60 & & & & & & & & & 694 & 7 & 0.96 & 0.02 & 1.9 & 0.1 \\
\hline $25 X-1$ & 195.10 & 195.20 & 16.1 & 0.6 & 388 & 1 & 923 & 5 & 0.78 & 0.04 & 706 & 5 & 0.87 & 0.01 & 1.6 & 0.1 \\
\hline 340-U139 & & & & & & & & & & & & & & & & \\
\hline $1 \mathrm{H}-3$ & 4.40 & 4.50 & 31.1 & 0.6 & 509 & 1 & 497 & 3 & 0.40 & 0.04 & 248.2 & 5 & 1.46 & 0.01 & 1.9 & 0.1 \\
\hline $2 \mathrm{H}-3$ & 12.80 & 12.90 & 34.0 & 0.6 & 510 & 3 & 573 & 3 & 0.56 & 0.05 & 242.7 & 5 & 1.28 & 0.02 & 1.6 & 0.1 \\
\hline $2 \mathrm{H}-6$ & 17.30 & 17.40 & 33.6 & 0.6 & 527 & 2 & 643 & 5 & 0.83 & 0.05 & 222.9 & 5 & 1.16 & 0.01 & 1.3 & 0.1 \\
\hline $3 \mathrm{H}-3$ & 22.20 & 22.30 & 33.5 & 0.6 & 522 & 3 & 629 & 3 & 0.77 & 0.04 & 206.7 & 5 & 1.12 & 0.02 & 1.1 & 0.1 \\
\hline $3 \mathrm{H}-6$ & 26.80 & 26.90 & 33.6 & 0.6 & 529 & 4 & 649 & 5 & 0.90 & 0.04 & 194.0 & 5 & 1.10 & 0.02 & 1.3 & 0.1 \\
\hline $4 \mathrm{H}-3$ & 31.80 & 31.90 & 33.7 & 0.6 & 541 & 5 & 702 & 3 & 1.08 & 0.04 & 181 & 1 & 1.10 & 0.01 & 1.2 & 0.1 \\
\hline $4 \mathrm{H}-5$ & 34.80 & 34.90 & 33.3 & 0.6 & 527 & 1 & 647 & 4 & 1.38 & 0.04 & 183 & 1 & 1.10 & 0.01 & 1.2 & 0.1 \\
\hline $5 \mathrm{H}-4$ & 42.80 & 42.90 & 33.7 & 0.6 & 536 & 2 & 714 & 4 & 1.29 & 0.04 & 166 & 2 & 1.11 & 0.02 & 1.3 & 0.2 \\
\hline $5 \mathrm{H}-6$ & 45.80 & 45.90 & 34.0 & 0.6 & 535 & 3 & 713 & 5 & 1.74 & 0.04 & 164 & 1 & 1.10 & 0.01 & 1.2 & 0.2 \\
\hline $6 \mathrm{H}-3$ & 50.70 & 50.80 & 33.8 & 0.6 & 529 & 3 & 725 & 3 & 1.96 & 0.05 & 158 & 1 & 1.11 & 0.02 & 1.3 & 0.1 \\
\hline $6 \mathrm{H}-6$ & 55.30 & 55.40 & 33.4 & 0.6 & 532 & 2 & 772 & 4 & 2.32 & 0.04 & 155 & 2 & 1.12 & 0.01 & 1.2 & 0.1 \\
\hline $7 \mathrm{H}-3$ & 60.30 & 60.40 & 33.0 & 0.6 & 533 & 6 & 744 & 6 & 2.71 & 0.05 & 155 & 1 & 1.12 & 0.02 & 1.2 & 0.1 \\
\hline $7 \mathrm{H}-6$ & 64.80 & 64.90 & 32.5 & 0.6 & 533 & 4 & 754 & 4 & 3.08 & 0.05 & 148 & 1 & 1.10 & 0.01 & 1.4 & 0.1 \\
\hline $8 \mathrm{H}-3$ & 69.80 & 69.90 & 32.0 & 0.6 & 548 & 3 & 753 & 4 & 3.54 & 0.05 & 145 & 1 & 1.11 & 0.02 & 1.3 & 0.2 \\
\hline $8 \mathrm{H}-6$ & 74.35 & 74.45 & 32.3 & 0.6 & 545 & 2 & 753 & 3 & 4.39 & 0.04 & 144 & 1 & 1.14 & 0.01 & 1.6 & 0.1 \\
\hline $9 \mathrm{H}-3$ & 79.30 & 79.40 & 31.9 & 0.6 & 545 & 4 & 770 & 4 & 6.92 & 0.05 & 143 & 1 & 1.15 & 0.02 & 1.7 & 0.1 \\
\hline $9 \mathrm{H}-5$ & 82.30 & 82.40 & 31.7 & 0.6 & 539 & 4 & 766 & 4 & 9.59 & 0.11 & 141 & 1 & 1.13 & 0.02 & 1.7 & 0.1 \\
\hline $10 \mathrm{H}-3$ & 88.82 & 88.92 & 31.8 & 0.6 & 529 & 3 & 726 & 3 & 8.32 & 0.05 & 142 & 1 & 1.09 & 0.02 & 1.7 & 0.2 \\
\hline $10 \mathrm{H}-6$ & 93.35 & 93.45 & 31.6 & 0.6 & 521 & 3 & 739 & 3 & 9.20 & 0.06 & 142 & 1 & 1.06 & 0.01 & 1.9 & 0.2 \\
\hline $11 \mathrm{H}-3$ & 98.30 & 98.40 & 31.8 & 0.6 & 521 & 5 & 718 & 3 & 10.37 & 0.05 & 143 & 1 & 1.13 & 0.02 & 2.4 & 0.1 \\
\hline $11 \mathrm{H}-6$ & 102.71 & 102.81 & 31.2 & 0.6 & 517 & 2 & 726 & 4 & 12.10 & 0.08 & 141 & 1 & 1.23 & 0.02 & 2.6 & 0.2 \\
\hline $12 \mathrm{H}-3$ & 107.83 & 107.93 & 31.0 & 0.6 & 512 & 3 & 721 & 4 & 14.48 & 0.08 & 140 & 1 & 1.19 & 0.02 & 2.7 & 0.1 \\
\hline $12 \mathrm{H}-6$ & 112.10 & 112.20 & 30.9 & 0.6 & 508 & 3 & 704 & 3 & 15.40 & 0.05 & 139 & 1 & 1.15 & 0.01 & 2.6 & 0.1 \\
\hline $13 \mathrm{H}-2$ & 115.81 & 115.91 & 30.9 & 0.6 & 517 & 4 & 720 & 2 & 18.17 & 0.07 & 139 & 1 & 1.15 & 0.02 & 3.2 & 0.2 \\
\hline $14 \mathrm{H}-5$ & 127.49 & 127.59 & 30.6 & 0.6 & 512 & 3 & 767 & 3 & 22.57 & 0.10 & 136 & 1 & 1.16 & 0.01 & 3.6 & 0.2 \\
\hline $15 \mathrm{H}-3$ & 134.33 & 134.43 & 30.1 & 0.6 & 489 & 2 & 780 & 3 & 23.23 & 0.06 & 132 & 1 & 1.12 & 0.02 & 3.8 & 0.2 \\
\hline $15 \mathrm{H}-6$ & 138.80 & 138.90 & 30.6 & 0.6 & 493 & 1 & 778 & 4 & 24.58 & 0.18 & 134 & 1 & 1.11 & 0.01 & 3.5 & 0.2 \\
\hline
\end{tabular}


Table T1 (continued).

\begin{tabular}{|c|c|c|c|c|c|c|c|c|c|c|c|c|c|c|c|c|}
\hline \multirow{2}{*}{$\begin{array}{l}\text { Core, } \\
\text { section }\end{array}$} & \multicolumn{2}{|c|}{ Depth CSF-A (m) } & \multirow{2}{*}{$\begin{array}{c}\mathrm{Li} \\
(\mu \mathrm{M})\end{array}$} & \multirow{2}{*}{$\begin{array}{c} \pm \\
(\mu \mathrm{M})\end{array}$} & \multirow{2}{*}{$\begin{array}{c}B \\
(\mu \mathrm{M})\end{array}$} & \multirow{2}{*}{$\stackrel{ \pm}{(\mu \mathrm{M})}$} & \multirow{2}{*}{$\begin{array}{c}\mathrm{Si} \\
(\mu \mathrm{M})\end{array}$} & \multirow{2}{*}{$\begin{array}{c} \pm \\
(\mu \mathrm{M})\end{array}$} & \multirow{2}{*}{$\begin{array}{c}\mathrm{Mn} \\
(\mu \mathrm{M})\end{array}$} & & $\mathrm{Sr}$ & \pm & $\mathrm{Rb}$ & & $\mathrm{Cs}$ & \\
\hline & Top & Bottom & & & & & & & & $(\mu \mathrm{M})$ & $(\mu \mathrm{M})$ & $(\mu \mathrm{M})$ & $(\mu \mathrm{M})$ & $(\mu \mathrm{M})$ & $(\mathrm{pM})$ & $(\mathrm{pM})$ \\
\hline $340-U 135$ & & & & & & & & & & & & & & & & \\
\hline $1 \mathrm{H}-2$ & 2.90 & 3.00 & 30.6 & 1.3 & 486 & 2 & 541 & 6 & 43.3 & 0.2 & 99 & 1 & 1.70 & 0.04 & 1.62 & 0.19 \\
\hline $2 \mathrm{H}-3$ & 10.10 & 10.20 & 28.7 & 1.3 & 491 & 3 & 500 & 6 & 41.5 & 0.3 & 86 & 1 & 1.60 & 0.02 & 2.09 & 0.13 \\
\hline $3 \mathrm{H}-3$ & 19.62 & 19.72 & 26.8 & 1.3 & 485 & 5 & 550 & 7 & 46.3 & 0.3 & 85 & 1 & 1.61 & 0.01 & 2.17 & 0.13 \\
\hline $3 \mathrm{H}-5$ & 22.60 & 22.70 & & & & & & & & & & & 1.53 & 0.02 & 1.77 & 0.16 \\
\hline $4 \mathrm{H}-2$ & 27.60 & 27.70 & 25.2 & 1.3 & 490 & 2 & 596 & 6 & 52.6 & 0.2 & 82 & 1 & 1.32 & 0.01 & 1.75 & 0.13 \\
\hline $4 \mathrm{H}-4$ & 30.60 & 30.70 & 25.1 & 1.3 & 476 & 1 & 583 & 7 & 44.8 & 0.3 & 79 & 1 & 1.36 & 0.02 & 1.88 & 0.12 \\
\hline $5 \mathrm{H}-3$ & 38.60 & 38.70 & 26.3 & 1.3 & 448 & 3 & 637 & 6 & 22.7 & 0.1 & 77 & 1 & 1.54 & 0.02 & 2.21 & 0.12 \\
\hline $5 \mathrm{H}-5$ & 41.60 & 41.70 & 27.1 & 1.3 & 483 & 3 & 703 & 5 & 26.2 & 0.1 & 75 & 1 & 1.49 & 0.03 & 2.10 & 0.14 \\
\hline $6 \mathrm{H}-2$ & 46.50 & 46.60 & 26.5 & 1.3 & 453 & 5 & 685 & 7 & 23.3 & 0.1 & 72 & 1 & 1.41 & 0.01 & 1.97 & 0.12 \\
\hline $6 \mathrm{H}-4$ & 49.45 & 49.55 & 27.3 & 1.2 & 462 & 2 & 675 & 5 & 21.2 & 0.1 & 72 & 1 & 1.48 & 0.02 & 2.25 & 0.13 \\
\hline $8 \mathrm{H}-1$ & 64.12 & 64.22 & 27.0 & 1.3 & 437 & 3 & 612 & 5 & 17.4 & 0.1 & 68 & 1 & 1.45 & 0.02 & 2.31 & 0.14 \\
\hline $8 \mathrm{H}-3$ & 67.07 & 67.17 & 27.1 & 1.2 & 454 & 1 & 682 & 6 & 19.2 & 0.1 & 67 & 1 & 1.43 & 0.03 & 2.25 & 0.14 \\
\hline $9 \mathrm{H}-3$ & 76.32 & 76.42 & 27.4 & 1.3 & 460 & 3 & 674 & 6 & 16.6 & 0.1 & 63 & 1 & 1.37 & 0.02 & 2.33 & 0.17 \\
\hline $12 \mathrm{H}-1$ & 95.72 & 95.82 & 29.5 & 1.3 & 448 & 3 & 716 & 5 & 13.4 & 0.1 & 66 & 1 & 1.43 & 0.02 & 2.66 & 0.19 \\
\hline $13 \mathrm{H}-3$ & 107.10 & 107.20 & 28.7 & 1.3 & 411 & 2 & 655 & 6 & 13.5 & 0.1 & 62 & 1 & 1.47 & 0.02 & 2.71 & 0.18 \\
\hline $15 \mathrm{H}-5$ & 122.13 & 122.24 & 29.1 & 1.2 & 390 & 3 & 756 & 5 & 14.9 & 0.1 & 61 & 1 & 1.28 & 0.02 & 2.32 & 0.14 \\
\hline $18 \mathrm{H}-2$ & 131.83 & 131.93 & 28.5 & 1.2 & 399 & 2 & 671 & 6 & 14.9 & 0.1 & 61 & 1 & 1.36 & 0.02 & 2.70 & 0.15 \\
\hline $24 \mathrm{H}-1$ & 160.70 & 160.80 & 37.3 & 1.2 & 380 & 3 & 690 & 6 & 8.6 & 0.1 & 63 & 1 & 1.41 & 0.02 & 2.87 & 0.13 \\
\hline $25 \mathrm{H}-1$ & 167.80 & 167.90 & 38.2 & 1.3 & 376 & 3 & 732 & 5 & 10.5 & 0.1 & 62 & 1 & 1.33 & 0.01 & 2.76 & 0.12 \\
\hline 340-U14C & & & & & & & & & & & & & & & & \\
\hline 7H-4 & 31.24 & 31.34 & 29.4 & 1.3 & 471 & 5 & 612 & 7 & 19.0 & 0.1 & 88 & 1 & 1.81 & 0.06 & 2.27 & 0.15 \\
\hline $8 \mathrm{H}-5$ & 42.40 & 42.50 & 29.1 & 1.3 & 453 & 2 & 605 & 7 & 27.3 & 0.1 & 88 & 1 & 1.64 & 0.02 & 2.33 & 0.15 \\
\hline $9 \mathrm{H}-1$ & 45.90 & 46.00 & 27.9 & 1.3 & 469 & 4 & 564 & 6 & 31.6 & 0.2 & 89 & 1 & 1.58 & 0.02 & 2.57 & 0.15 \\
\hline $10 \mathrm{H}-3$ & 52.91 & 53.01 & 28.8 & 1.3 & 459 & 6 & 549 & 7 & 33.6 & 0.2 & 91 & 1 & 1.61 & 0.01 & 2.65 & 0.13 \\
\hline $10 \mathrm{H}-6$ & 57.42 & 57.52 & 28.9 & 1.3 & 462 & 4 & 660 & 6 & 32.4 & 0.2 & 93 & 1 & 1.52 & 0.02 & 2.52 & 0.12 \\
\hline $11 \mathrm{H}-4$ & 63.92 & 64.02 & 29.8 & 1.2 & 422 & 3 & 687 & 7 & 33.7 & 0.1 & 93 & 1 & 1.64 & 0.02 & 3.87 & 0.12 \\
\hline $12 \mathrm{H}-2$ & 70.08 & 70.18 & 30.0 & 1.2 & 433 & 4 & 673 & 6 & 30.6 & 0.1 & 93 & 1 & 1.60 & 0.01 & 4.27 & 0.13 \\
\hline $13 \mathrm{H}-5$ & 81.40 & 81.50 & 33.0 & 1.3 & 405 & 2 & 689 & 6 & 30.9 & 0.1 & 98 & 1 & 1.67 & 0.02 & 4.70 & 0.12 \\
\hline $14 \mathrm{H}-3$ & 87.85 & 87.95 & 32.9 & 1.2 & 403 & 2 & 738 & 6 & 30.3 & 0.1 & 97 & 1 & 1.65 & 0.02 & 4.49 & 0.17 \\
\hline $15 \mathrm{H}-4$ & 98.80 & 98.90 & 32.0 & 1.3 & 388 & 3 & 751 & 7 & 32.0 & 0.3 & 98 & 1 & 1.53 & 0.02 & 3.47 & 0.13 \\
\hline $17 \mathrm{H}-4$ & 111.53 & 111.63 & 33.2 & 1.2 & 365 & 3 & 770 & 6 & 31.8 & 0.2 & 100 & 1 & 1.53 & 0.02 & 3.86 & 0.19 \\
\hline $18 \mathrm{H}-4$ & 121.00 & 121.10 & 32.7 & 1.3 & 369 & 2 & 758 & 6 & 32.5 & 0.1 & 101 & 1 & 1.54 & 0.02 & 3.85 & 0.16 \\
\hline $19 \mathrm{H}-2$ & 127.52 & 127.62 & 32.4 & 1.2 & 354 & 3 & 727 & 5 & 32.0 & 0.1 & 103 & 1 & 1.44 & 0.02 & 3.24 & 0.14 \\
\hline $20 \mathrm{H}-3$ & 133.21 & 133.31 & 32.0 & 1.3 & 354 & 2 & 762 & 6 & 32.9 & 0.2 & 102 & 1 & 1.45 & 0.01 & 3.07 & 0.13 \\
\hline $21 \mathrm{H}-5$ & 143.82 & 143.92 & 32.6 & 1.2 & 336 & 2 & 834 & 6 & 31.6 & 0.1 & 105 & 1 & 1.39 & 0.01 & 2.52 & 0.12 \\
\hline $22 \mathrm{H}-4$ & 151.83 & 151.93 & 32.0 & 1.3 & 324 & 2 & 818 & 8 & 28.8 & 0.1 & 104 & 1 & 1.34 & 0.01 & 2.27 & 0.12 \\
\hline $23 \mathrm{H}-4$ & 161.35 & 161.45 & 37.2 & 1.2 & 368 & 3 & 905 & 10 & 30.2 & 0.3 & 124 & 1 & 1.32 & 0.02 & 2.24 & 0.15 \\
\hline $24 \mathrm{H}-4$ & 170.88 & 170.98 & 31.3 & 1.3 & 332 & 3 & 713 & 6 & 24.2 & 0.2 & 105 & 1 & 1.20 & 0.01 & 1.81 & 0.12 \\
\hline $25 \mathrm{H}-4$ & 180.34 & 180.44 & 31.3 & 1.2 & 341 & 1 & 599 & 6 & 20.8 & 0.1 & 103 & 1 & 1.13 & 0.01 & 1.99 & 0.12 \\
\hline $26 \mathrm{H}-4$ & 189.78 & 189.88 & 33.4 & 1.3 & 333 & 2 & 843 & 6 & 28.8 & 0.2 & 103 & 1 & 1.39 & 0.02 & 3.40 & 0.16 \\
\hline $27 \mathrm{H}-5$ & 200.79 & 200.89 & 34.2 & 1.2 & 342 & 2 & 754 & 6 & 29.5 & 0.1 & 104 & 1 & 1.66 & 0.03 & 3.82 & 0.13 \\
\hline $28 \mathrm{H}-3$ & 207.30 & 207.40 & 34.4 & 1.3 & 366 & 4 & 867 & 9 & 34.0 & 0.2 & 104 & 1 & 1.56 & 0.02 & 3.62 & 0.15 \\
\hline 340-U140 & & & & & & & & & & & & & & & & \\
\hline $27 X-4$ & 231.20 & 231.30 & 33.8 & 1.2 & 328 & 2 & 748 & 6 & 33.6 & 0.1 & 101 & 1 & 1.48 & 0.01 & 3.74 & 0.11 \\
\hline $29 X-2$ & 247.40 & 247.50 & 34.6 & 1.3 & 323 & 2 & 846 & 6 & 33.7 & 0.3 & 104 & 1 & 1.39 & 0.02 & 3.25 & 0.19 \\
\hline $30 X-2$ & 257.00 & 257.10 & 35.3 & 1.3 & 351 & 2 & 790 & 6 & 31.7 & 0.1 & 104 & 1 & 1.49 & 0.02 & 3.38 & 0.12 \\
\hline $31 X-3$ & 268.10 & 268.20 & 35.1 & 1.3 & 337 & 2 & 833 & 9 & 30.2 & 0.2 & 109 & 1 & 1.46 & 0.01 & 3.04 & 0.13 \\
\hline $32 X-2$ & 276.20 & 276.30 & 35.0 & 1.3 & 355 & 4 & 837 & 11 & 29.0 & 0.2 & 112 & 1 & 1.47 & 0.02 & 3.05 & 0.13 \\
\hline $33 X-2$ & 285.80 & 285.90 & 33.2 & 1.3 & 336 & 2 & 688 & 6 & 18.9 & 0.1 & 111 & 1 & 1.33 & 0.01 & 2.62 & 0.16 \\
\hline $35 X-4$ & 298.40 & 298.50 & 32.0 & 1.3 & 316 & 1 & 656 & 6 & 17.7 & 0.1 & 112 & 1 & 1.27 & 0.02 & 2.84 & 0.13 \\
\hline $36 X-3$ & 306.50 & 306.60 & 32.6 & 1.3 & 335 & 2 & 647 & 6 & 17.7 & 0.1 & 114 & 1 & 1.35 & 0.01 & 3.30 & 0.15 \\
\hline $37 X-1$ & 313.12 & 313.22 & 32.9 & 1.2 & 331 & 2 & 682 & 8 & 18.9 & 0.1 & 116 & 1 & 1.42 & 0.02 & 3.78 & 0.13 \\
\hline $38 X-5$ & 328.30 & 328.40 & 32.3 & 1.3 & 326 & 3 & 728 & 7 & 20.8 & 0.2 & 113 & 1 & 1.46 & 0.02 & 3.61 & 0.14 \\
\hline $39 X-5$ & 337.71 & 337.81 & 34.0 & 1.3 & 332 & 3 & 768 & 9 & 22.4 & 0.2 & 116 & 1 & 1.48 & 0.02 & 3.38 & 0.15 \\
\hline $40 X-1$ & 341.50 & 341.60 & 33.0 & 1.3 & 312 & 2 & 783 & 9 & 21.7 & 0.2 & 114 & 1 & 1.41 & 0.02 & 3.43 & 0.12 \\
\hline $41 \mathrm{X}-1$ & 351.10 & 351.20 & 33.2 & 1.2 & 304 & 2 & 689 & 6 & 23.6 & 0.1 & 115 & 1 & 1.11 & 0.01 & 2.02 & 0.12 \\
\hline $42 X-2$ & 362.20 & 362.30 & 32.6 & 1.3 & 306 & 4 & 902 & 7 & 21.9 & 0.2 & 114 & 1 & 1.39 & 0.02 & 2.96 & 0.20 \\
\hline $44 \mathrm{X}-1$ & 379.75 & 379.85 & 33.9 & 1.3 & 300 & 4 & 894 & 12 & 25.0 & 0.2 & 115 & 1 & 1.33 & 0.03 & 2.39 & 0.14 \\
\hline $45 X-3$ & 392.45 & 392.55 & 38.9 & 1.2 & 304 & 2 & 941 & 6 & 30.4 & 0.2 & 114 & 1 & 1.40 & 0.02 & 2.67 & 0.14 \\
\hline $49 X-1$ & 427.48 & 427.58 & 50.3 & 1.3 & 308 & 2 & 679 & 6 & 32.2 & 0.2 & 131 & 1 & 1.01 & 0.02 & 1.86 & 0.16 \\
\hline
\end{tabular}

Sr values in bold are from inductively coupled plasma-optical emission spectrometry analysis at a higher dilution, as indicated in text. 
Table T2. Composition of sediment digest, Sites U1395-U1400.

\begin{tabular}{|c|c|c|c|c|c|c|c|c|c|c|c|c|c|c|c|c|c|c|c|c|c|c|c|c|c|c|c|c|}
\hline \multirow{2}{*}{$\begin{array}{l}\text { Core, } \\
\text { section }\end{array}$} & \multicolumn{2}{|c|}{ Offset $(\mathrm{cm})$} & \multicolumn{2}{|c|}{ Depth CSF-A $(\mathrm{m})$} & \multirow{2}{*}{$\stackrel{\mathrm{Al}}{\mathrm{Al}}$} & \multirow{2}{*}{$\underset{\text { (wt\%) }}{ \pm}$} & \multirow{2}{*}{$\begin{array}{c}\mathrm{Ba} \\
(\mathrm{ppm})\end{array}$} & \multirow{2}{*}{$\begin{array}{c}\stackrel{ \pm}{(p p m)} \\
\text { (pons }\end{array}$} & \multirow{2}{*}{$\begin{array}{c}\mathrm{Ca} \\
\text { (wt\%) }\end{array}$} & \multirow{2}{*}{$\underset{(w t \%)}{ \pm}$} & \multirow{2}{*}{$\begin{array}{c}\mathrm{Cu} \\
(\mathrm{ppm})\end{array}$} & \multirow{2}{*}{$\begin{array}{c} \pm \\
(\mathrm{ppm})\end{array}$} & \multirow{2}{*}{$\begin{array}{c}\mathrm{Fe} \\
(\text { wt\%) }\end{array}$} & & & & & & & & & & & & & & & \\
\hline & Top & Bottom & Top & Bottom & & & & & & & & & & & & & & & & & & & ( & & ) $(1$ & & & \\
\hline 4136 & & & & & & & & & & & & & & & & & & & & & & & & & & & & \\
\hline & 140 & 150 & 4.40 & 50 & .18 & & 79 & 5 & 29.3 & 0.1 & 12.4 & 1.2 & 1.21 & 0.02 & 0.23 & 0.04 & 1.24 & 0.01 & 299 & 4 & 0.87 & 0.01 & 2272 & 8 & 0.10 & 0.00 & 42.5 & \\
\hline & 130 & & & & & & 163 & 4 & & & & & & & & & & & & & & 01 & & & & & & \\
\hline & & & & & & & 21 & 15 & & & & & & & & & & & & & & & & & & & & \\
\hline $14 \mathrm{H}$ & 14 & 150 & & & & & 192 & 15 & & & & & & & & & & & & & & & & & & & & .5 \\
\hline $14 \mathrm{H}-3$ & 14 & & 7.30 & 7.40 & & & 195 & 15 & & & & & & & 0. & & 1. & & & 7 & & & & 3 & & 0.00 & & 1.6 \\
\hline & 14 & & & & & & 98 & 5 & 27 & & & & & & & & & & & 5 & & & & 10 & & 00 & & 2.2 \\
\hline $25 \mathrm{X}-1$ & 140 & 15 & 5.10 & 5.20 & & & 245 & 15 & 22 & 0 & 23.4 & 1 & & 02 & 0.67 & .03 & 0.8 & 0.01 & & 5 & 0.97 & 0.01 & 2267 & 14 & 0.17 & 0.00 & 64.5 & 2.4 \\
\hline 340-U13s & & & & & & & & & & & & & & & & & & & & & & & & & & & & \\
\hline+-3 & & & 4.40 & 4.50 & 3.63 & .03 & 161 & 4 & 26.1 & 0.1 & 14.4 & 1.3 & 1.98 & 0.02 & 0.38 & 0.05 & 0.64 & 0.01 & 339 & 4 & 1.15 & 0.01 & 1709 & 8 & 0.16 & 0.00 & 84.4 & 1.5 \\
\hline $2 \mathrm{H}-3$ & 140 & 150 & 12.80 & 12.90 & 4.19 & & 132 & 5 & 24.0 & 0.1 & 13.4 & 1.3 & & & 0.43 & & 0.66 & 0.01 & 497 & 5 & 1.26 & & & 7 & 0.16 & 0.00 & 4.3 & 1.8 \\
\hline & 140 & & & 12.90 & & & 130 & 4 & & 0. & & & & & 0. & & 0. & & & 3 & & & & 8 & & & 1.8 & 2.0 \\
\hline & & & & & & & & 15 & & & & & & & & & & & & & & & & 8 & & & & \\
\hline & & & & & & & & & & & & & & & & & & & & & & & & & & & & \\
\hline & & & & & & & & 5 & & & & & & & & & & & & & & & & & & & & \\
\hline & 14 & & & & & & & 4 & & & & & & & & & & & & & & & & & & & & .9 \\
\hline $15 \mathrm{H}-6$ & 140 & 150 & 138.80 & 38.90 & & .07 & 190 & 4 & 14.2 & 0.1 & 32.3 & 1.1 & 3.65 & 0.04 & 0.84 & 0.03 & 0.80 & .01 & 1023 & $\varepsilon$ & & & 601 & 7 & 32 & 0.00 & 102.6 & 1.5 \\
\hline $340-\mathrm{U1}$ & & & & & & & & & & & & & & & & & & & & & & & & & & & & \\
\hline & 140 & & & & & & 376 & 15 & 4.2 & 0.0 & 91.4 & 1.2 & & & & & & & & 5 & 2.64 & & & 2 & 0.17 & 0.00 & 52.8 & 1.5 \\
\hline & 14 & & & 10.20 & & & 307 & 14 & 9.9 & 0 & & & & & & & & 02 & 10 & 6 & & & & 8 & 34 & 00 & 0.0 & 2.3 \\
\hline & & & & & & & 26 & 1 & & 0 & & & & & & & & & & 5 & & & & 3 & & & & 1.7 \\
\hline & 13 & & & & & & 265 & 13 & 13 & & & & & & 1. & & & & & $\varepsilon$ & & & & 9 & & & & 1.6 \\
\hline & 13 & & & & & & & 1 & & & & & & & & & & & & & & & & & & & & 1.5 \\
\hline & & & & & & & & & & & & & & & & & & & & & & & & & & & & 1.6 \\
\hline & & & & & & & & & & & & & & & & & & & & & & & & & & & & \\
\hline $25 \mathrm{H}-1$ & 140 & 150 & 167.80 & 167.90 & 7.15 & 0.08 & 292 & 15 & 12.0 & & 45.8 & 1.2 & & 0.04 & 1.27 & 0.02 & 1.43 & & 1946 & 15 & & & 844 & 9 & 0.31 & 0.00 & 123.5 & 1.5 \\
\hline & & & & & & & & & & & & & & & & & & & & & & & & & & & & \\
\hline & 14 & & & & & & & 1 & & & & & & & & & & & & 2 & & & & & & & & 1.7 \\
\hline & & & & & & & & & & & & & & & & & & & & s & & & & & & & & \\
\hline & & & & & & & & & & & & & & & & & & & & . & & & & & & & & \\
\hline $28 \mathrm{H}-3$ & 137 & 147 & 207.30 & 207.40 & & & 276 & 15 & 7.3 & 0. & & 1 & & & 1.06 & & 1.45 & 0.02 & 1446 & 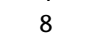 & 2.09 & 0.02 & 428 & 2 & 0.32 & 0.00 & 125.2 & 1.9 \\
\hline $0-11$ & & & & & & & & & & & & & & & & & & & & & & & & & & & & \\
\hline & & & & & & & & 4 & & & & & & & & & & & & 7 & & & & 8 & & & & \\
\hline & 14 & & & & & & 290 & 15 & & & & & & & & & & & & 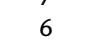 & & & & & & & 28.4 & 1.4 \\
\hline $49 \mathrm{X}-1$ & 108 & 116 & 427.48 & 427.58 & 8.10 & 0.08 & 293 & 16 & 8.5 & 0.1 & 48.4 & 1.3 & 4.01 & 0.06 & 1.68 & 0.02 & 1.31 & 0.02 & 1264 & 16 & 1.27 & 0.02 & 514 & 5 & 0.38 & 0.00 & 147.7 & 1.6 \\
\hline & & 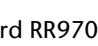 & $-42 \mathrm{MC} C \mathrm{C}$ & 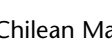 & 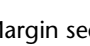 & edin & & & & & & & & & & & & & & & & & & & & & & \\
\hline & & & & & 8.1 & 0.04 & 406 & 2 & $\begin{array}{c}2.66 \\
5\end{array}$ & 0.04 & 40.8 & 0.5 & $\begin{array}{c}4.73 \\
5\end{array}$ & 0.05 & $\begin{array}{l}1.41 \\
5\end{array}$ & 0.01 & 1.45 & 0.02 & $\begin{array}{r}518 \\
5\end{array}$ & 5 & 3.21 & 0.03 & $\begin{array}{r}307 \\
5\end{array}$ & 3 & 0.48 & 0.004 & $\begin{array}{r}124 \\
5\end{array}$ & 1 \\
\hline & & & & & $\begin{array}{l}5 \\
81\end{array}$ & & 410 & 6 & & & & & & & & & & & $\begin{array}{r}5 \\
512\end{array}$ & & 3.22 & & $\begin{array}{r}5 \\
309\end{array}$ & 5 & 0.48 & .007 & $\begin{array}{r}5 \\
124\end{array}$ & \\
\hline & & & mber of sar & $\begin{array}{l}\text { bry results } \\
\text { mples }(N)\end{array}$ & $\begin{array}{l}8.18 \\
38\end{array}$ & & $\begin{array}{r}410 \\
38\end{array}$ & 6 & $\begin{array}{l}2.66 \\
38\end{array}$ & 0.04 & $\begin{array}{c}42.5 \\
38\end{array}$ & & $\begin{array}{l}4.9 \\
38\end{array}$ & & $\begin{array}{l}1.44 \\
32\end{array}$ & & $\begin{array}{l}1.43 \\
38\end{array}$ & & $\begin{array}{r}12 \\
38\end{array}$ & & $\begin{array}{l}3.22 \\
32\end{array}$ & & $\begin{array}{r}09 \\
38\end{array}$ & & $\begin{array}{l}0.40 \\
38\end{array}$ & & 38 & \\
\hline & & & & & & & & & & & & & & & & & & & & & & & & & & & & \\
\hline & & & & & 6.30 & 0.05 & 980 & 24 & 1.97 & 0.03 & NR & NR & & 0.04 & & 0.02 & 1.42 & 0.01 & 436 & 5 & 3.38 & 0.0 & 271 & 3 & 0.43 & 0.00 & 132 & 2 \\
\hline & & & & & & & 980 & & & & & & & & & & & & & & & & $\begin{array}{r}5 \\
73\end{array}$ & & & & 5 & \\
\hline & & & ber of s & ple & $\begin{array}{l}6.3 \\
38\end{array}$ & & $\begin{array}{r}980 \\
38\end{array}$ & 21 & 98 & & $\begin{aligned} 31 \\
2\end{aligned}$ & & $\begin{array}{l}\begin{array}{l}4.12 \\
38\end{array} \\
\text { a }\end{array}$ & & $\begin{array}{l}1.22 \\
32\end{array}$ & & $\begin{array}{l}1.41 \\
38\end{array}$ & & $\begin{array}{r}431 \\
38\end{array}$ & 7 & $\begin{array}{l}3.4 \\
32\end{array}$ & & $\begin{array}{r}273 \\
38\end{array}$ & 4 & $\begin{array}{l}0.43 \\
38\end{array}$ & & $\begin{array}{r}132 \\
38\end{array}$ & \\
\hline & & & Certifii & ied values & 6.62 & 0.16 & & & 1.96 & 0.09 & 310 & 6 & 4.09 & 0.03 & 1.24 & 0.03 & 1.47 & 0.07 & 440 & 10 & 3.45 & 0.09 & 276 & 15 & 0.443 & 0.016 & 133 & \\
\hline
\end{tabular}


Table T3. Inductively coupled plasma-optical emission spectrometry specifications.

\begin{tabular}{lcl}
\hline Element & Line (nm) & View \\
\hline $\mathrm{Al}$ & 396.152 & Radial \\
$\mathrm{Ba}$ & 455.403 & Radial \\
$\mathrm{Ca}$ & 317.933 & Radial \\
$\mathrm{Cu}$ & 324.754 & Axial \\
$\mathrm{Fe}$ & 259.940 & Radial \\
$\mathrm{K}$ & 766.491 & Radial \\
$\mathrm{Mg}$ & 279.078 & Axial \\
$\mathrm{Mn}$ & 257.610 & Radial \\
$\mathrm{Na}$ & 589.592 & Radial \\
$\mathrm{Sr}$ & 407.771 & Radial \\
$\mathrm{Ti}$ & 334.941 & Radial \\
$\mathrm{V}$ & 292.401 & Axial \\
$\mathrm{Zn}$ & 206.200 & Axial \\
\hline & &
\end{tabular}


Table T4. Composition of solid phases, Sites U1394-U1396, U1399, and U1400. (Continued on next two pages.)

\begin{tabular}{|c|c|c|c|c|c|c|c|c|c|c|c|c|c|c|}
\hline \multirow{2}{*}{$\begin{array}{l}\text { Core, } \\
\text { section }\end{array}$} & \multicolumn{2}{|c|}{ Offset (cm) } & \multicolumn{2}{|c|}{ Depth CSF-A (m) } & \multirow{2}{*}{$\begin{array}{l}\mathrm{CaCO}_{3} \\
\text { (wt\%) }\end{array}$} & \multirow{2}{*}{$\begin{array}{c} \pm \\
\text { (wt\%) }\end{array}$} & \multirow{2}{*}{$\begin{array}{c}\text { TIC } \\
\text { (wt\%) }\end{array}$} & \multirow{2}{*}{$\begin{array}{c} \pm \\
\text { (wt\%) }\end{array}$} & \multirow{2}{*}{$\begin{array}{c}\mathrm{OC} \\
(\mathrm{wt} \%)\end{array}$} & $\mathrm{N}$ & $\mathrm{Fe}_{\mathrm{R}}$ & \pm & $\mathrm{Mn}_{\mathrm{R}}$ & \pm \\
\hline & Top & Bottom & Top & Bottom & & & & & & (wt\%) & (wt\%) & $(w t \%)$ & (wt\%) & (wt\%) \\
\hline $340-U 139$ & & & & & & & & & & & & & & \\
\hline $1 \mathrm{H}-2$ & 94 & 95 & 2.44 & 2.45 & & & & & & & 0.34 & & 0.010 & \\
\hline $1 \mathrm{H}-4$ & 24 & 25 & 4.24 & 4.25 & 69.0 & & 8.29 & & 0.192 & 0.025 & 0.29 & & 0.044 & \\
\hline $2 \mathrm{H}-5$ & 81 & 82 & 11.67 & 11.68 & & & & & & & 0.69 & & 0.011 & \\
\hline $2 \mathrm{H}-7$ & 22 & 23 & 13.79 & 13.80 & 8.3 & & 1.00 & & 0.042 & 0.003 & 0.39 & & 0.008 & \\
\hline $3 \mathrm{H}-1$ & 37 & 38 & 14.67 & 14.68 & 67.3 & & 8.08 & & 0.152 & 0.022 & 0.33 & & 0.025 & 0.020 \\
\hline $3 \mathrm{H}-3$ & 43 & 44 & 17.73 & 17.74 & 34.1 & & 4.09 & & 0.050 & 0.006 & 0.42 & & 0.023 & \\
\hline $3 \mathrm{H}-6$ & 73 & 74 & 22.53 & 22.54 & 35.0 & & 4.20 & & 0.043 & 0.006 & 0.50 & & 0.018 & \\
\hline $14 \mathrm{X}-1$ & 80 & 81 & 111.20 & 111.21 & 62.6 & & 7.51 & & 0.190 & 0.025 & 0.33 & & 0.036 & \\
\hline $16 X-1$ & 48 & 49 & 130.08 & 130.09 & 12.5 & & 1.50 & & 0.210 & 0.026 & 0.36 & & 0.022 & \\
\hline $16 X-2$ & 63 & 64 & 131.69 & 131.70 & 58.5 & & 7.02 & & 0.267 & 0.032 & 0.35 & 0.23 & 0.022 & 0.029 \\
\hline $17 X-2$ & 47 & 48 & 141.17 & 141.18 & 58.2 & & 6.99 & & 0.292 & 0.029 & 0.37 & 0.09 & 0.026 & 0.009 \\
\hline $17 X-5$ & 122 & 123 & 146.42 & 146.43 & 59.0 & & 7.08 & & 0.355 & 0.036 & 0.30 & & 0.015 & \\
\hline $18 X-1$ & 44 & 45 & 149.24 & 149.25 & 37.2 & & 4.47 & & 0.115 & 0.018 & 0.37 & & 0.042 & \\
\hline $18 X-3$ & 71 & 72 & 152.51 & 152.52 & 56.4 & & 6.77 & & 0.300 & 0.033 & 0.41 & & 0.029 & \\
\hline $19 X-1$ & 52 & 53 & 158.92 & 158.93 & 61.2 & & 7.34 & & 0.155 & 0.021 & 0.37 & & 0.030 & \\
\hline $19 X-2$ & 67 & 68 & 160.57 & 160.58 & 55.2 & & 6.62 & & 0.420 & 0.043 & 0.36 & 0.01 & 0.019 & 0.000 \\
\hline $20 X-1$ & 57 & 58 & 168.57 & 168.58 & 32.1 & & 3.86 & & 0.113 & 0.016 & 0.44 & 0.01 & 0.035 & 0.011 \\
\hline $22 X-1$ & 21 & 22 & 187.31 & 187.32 & 54.5 & & 6.55 & & 0.183 & 0.024 & 0.35 & 0.01 & 0.043 & 0.001 \\
\hline $23 X-1$ & 68 & 69 & 197.38 & 197.39 & 50.9 & & 6.10 & & 0.213 & 0.024 & 0.36 & 0.01 & 0.035 & 0.001 \\
\hline $340-U 139$ & & & & & & & & & & & & & & \\
\hline $1 \mathrm{H}-2$ & 55 & 56 & 0.85 & 0.86 & 75.8 & & 9.10 & & 0.104 & 0.012 & 0.18 & & 0.007 & \\
\hline $2 \mathrm{H}-3$ & 104 & 105 & 7.14 & 7.15 & 34.9 & & 4.19 & & 0.184 & 0.024 & 0.37 & & 0.028 & \\
\hline $2 \mathrm{H}-6$ & 7 & 8 & 10.67 & 10.68 & 12.7 & & 1.53 & & 0.030 & 0.003 & 0.40 & 0.01 & 0.010 & 0.000 \\
\hline $4 \mathrm{H}-4$ & 96 & 97 & 25.36 & 25.37 & 45.1 & & 5.41 & & 0.094 & 0.015 & 0.71 & 0.01 & 0.031 & 0.000 \\
\hline $6 \mathrm{H}-2$ & 112 & 113 & 41.53 & 41.54 & 0.1 & & 0.02 & & 0.026 & 0.000 & 0.39 & 0.01 & 0.009 & \\
\hline $6 \mathrm{H}-5$ & 44 & 45 & 45.37 & 45.38 & 29.5 & & 3.54 & & 0.119 & 0.012 & 0.51 & 0.02 & 0.010 & 0.000 \\
\hline $6 \mathrm{H}-6$ & 91 & 92 & 47.35 & 47.36 & 12.2 & & 1.46 & & 0.108 & 0.013 & 0.48 & 0.01 & 0.018 & 0.000 \\
\hline $7 \mathrm{H}-1$ & 22 & 23 & 48.62 & 48.63 & 48.0 & & 5.76 & & 0.214 & 0.026 & 0.54 & 0.01 & 0.037 & 0.000 \\
\hline $7 \mathrm{H}-2$ & 135 & 136 & 51.25 & 51.26 & 29.5 & & 3.54 & & 0.138 & 0.012 & 0.50 & 0.01 & 0.009 & 0.000 \\
\hline $7 \mathrm{H}-6$ & 26 & 27 & 55.74 & 55.75 & 45.3 & & 5.43 & & 0.069 & 0.007 & 0.37 & 0.01 & 0.008 & 0.000 \\
\hline $8 \mathrm{H}-1$ & 18 & 19 & 57.28 & 57.29 & 46.7 & & 5.60 & & 0.253 & 0.031 & 0.51 & 0.03 & 0.020 & 0.001 \\
\hline $8 \mathrm{H}-3$ & 13 & 14 & 60.23 & 60.24 & 54.0 & & 6.49 & & 0.234 & 0.022 & 0.39 & 0.02 & 0.019 & 0.000 \\
\hline $9 \mathrm{H}-4$ & 134 & 135 & 68.77 & 68.78 & 9.8 & & 1.18 & & 0.087 & 0.008 & 0.75 & & 0.008 & \\
\hline $9 \mathrm{H}-6$ & 110 & 111 & 71.55 & 71.56 & 44.5 & & 5.34 & & 0.109 & 0.013 & 0.35 & & 0.019 & \\
\hline $10 \mathrm{H}-3$ & 67 & 68 & 76.07 & 76.08 & 1.9 & & 0.23 & & 0.053 & 0.004 & 0.51 & & 0.006 & \\
\hline $10 \mathrm{H}-4$ & 69 & 70 & 77.59 & 77.60 & 43.3 & & 5.19 & & 0.147 & 0.021 & 0.45 & & 0.032 & \\
\hline $11 \mathrm{H}-2$ & 103 & 104 & 82.93 & 82.94 & 54.5 & & 6.55 & & 0.209 & 0.012 & 0.39 & & 0.091 & \\
\hline $11 \mathrm{H}-6$ & 33 & 34 & 88.28 & 88.29 & 53.6 & & 6.43 & & 0.166 & 0.024 & 0.34 & & 0.039 & \\
\hline $12 \mathrm{H}-3$ & 130 & 131 & 94.20 & 94.21 & 37.4 & & 4.49 & & 0.064 & 0.008 & 0.24 & & 0.011 & \\
\hline $12 \mathrm{H}-4$ & 78 & 79 & 95.19 & 95.20 & 4.1 & & 0.49 & & 0.014 & 0.002 & 0.39 & 0.04 & 0.005 & 0.000 \\
\hline $12 \mathrm{H}-7$ & 46 & 47 & 99.38 & 99.39 & 35.7 & & 4.28 & & 0.082 & 0.014 & 0.45 & & 0.034 & \\
\hline $14 \mathrm{H}-1$ & 60 & 61 & 109.50 & 109.51 & 68.6 & & 8.24 & & 0.263 & 0.030 & 0.23 & & 0.013 & \\
\hline $14 \mathrm{H}-3$ & 16 & 17 & 112.06 & 112.07 & 43.5 & & 5.22 & & 0.093 & 0.011 & 0.20 & & 0.009 & \\
\hline $18 X-1$ & 39 & 40 & 135.99 & 136.00 & 55.4 & & 6.65 & & 0.239 & 0.031 & 0.33 & & 0.017 & \\
\hline $18 X-3$ & 58 & 59 & 139.18 & 139.20 & 5.7 & & 0.69 & & 0.166 & 0.013 & 0.70 & & 0.004 & \\
\hline $22 X-1$ & 39 & 40 & 169.29 & 169.30 & 81.1 & & 9.74 & & 0.148 & 0.016 & 0.21 & & 0.031 & \\
\hline $25 X-1$ & 49 & 50 & 193.49 & 193.50 & & & 0.00 & & & 0.000 & 0.21 & & 0.012 & \\
\hline $26 X-1$ & 59 & 60 & 203.19 & 203.20 & 62.3 & & 7.48 & & 0.169 & 0.021 & 0.38 & & 0.022 & \\
\hline $26 X-2$ & 6 & 7 & 204.09 & 204.10 & 65.5 & & 7.86 & & 0.103 & 0.012 & 0.36 & & 0.021 & \\
\hline $27 X-1$ & 48 & 49 & 212.58 & 212.60 & & & 0.00 & & & 0.000 & 0.28 & & 0.016 & \\
\hline $28 \times 1$ & 74 & 75 & 222.44 & 222.50 & 43.5 & & 5.22 & & 0.210 & 0.016 & 0.29 & & 0.013 & \\
\hline $340-U 139$ & & & & & & & & & & & & & & \\
\hline $1 \mathrm{H}-1$ & 77 & 78 & 0.77 & 0.78 & 59.6 & & 7.15 & & 0.089 & 0.015 & 0.37 & 0.01 & 0.011 & 0.000 \\
\hline $1 \mathrm{H}-4$ & 78 & 79 & 5.28 & 5.29 & 56.2 & & 6.75 & & 0.062 & 0.009 & 0.36 & & 0.013 & \\
\hline $3 \mathrm{H}-1$ & 120 & 121 & 16.30 & 16.31 & 42.3 & & 5.07 & & 0.074 & 0.011 & 0.43 & & 0.011 & \\
\hline $3 \mathrm{H}-4$ & 100 & 101 & 20.60 & 20.61 & 62.8 & & 7.54 & & 0.063 & 0.011 & 0.27 & 0.00 & 0.012 & 0.000 \\
\hline $3 \mathrm{H}-6$ & 56 & 57 & 23.16 & 23.17 & 72.4 & & 8.69 & & 0.072 & 0.012 & 0.32 & & 0.012 & \\
\hline $4 \mathrm{H}-2$ & 63 & 64 & 26.73 & 26.74 & 32.2 & & 3.86 & & 0.097 & 0.012 & 0.39 & & 0.007 & \\
\hline $4 \mathrm{H}-4$ & 95 & 96 & 30.05 & 30.06 & 60.4 & & 7.25 & & 0.153 & 0.015 & 0.32 & & 0.013 & \\
\hline $4 \mathrm{H}-6$ & 24 & 25 & 32.34 & 32.35 & 35.5 & & 4.26 & & 0.105 & 0.013 & 0.34 & & 0.009 & \\
\hline $5 \mathrm{H}-1$ & 51 & 52 & 34.61 & 34.62 & 13.4 & & 1.61 & & 0.032 & 0.004 & 0.40 & 0.01 & 0.005 & 0.000 \\
\hline $5 \mathrm{H}-4$ & 87 & 88 & 39.47 & 39.48 & 73.1 & & 8.77 & & 0.099 & 0.015 & 0.33 & & 0.012 & \\
\hline $6 \mathrm{H}-2$ & 97 & 97 & 46.07 & 46.08 & 0.0 & & 0.00 & & 0.000 & 0.000 & 0.34 & & 0.014 & \\
\hline $6 \mathrm{H}-6$ & 102 & 103 & 52.12 & 52.13 & 59.1 & & 7.10 & & 0.184 & 0.022 & 0.38 & & 0.011 & \\
\hline $7 \mathrm{H}-2$ & 89 & 90 & 55.49 & 55.50 & 53.2 & & 6.39 & & 0.149 & 0.018 & 0.37 & 0.02 & 0.014 & 0.001 \\
\hline
\end{tabular}


Table T4 (continued). (Continued on next page.)

\begin{tabular}{|c|c|c|c|c|c|c|c|c|c|c|c|c|c|c|}
\hline \multirow{2}{*}{$\begin{array}{c}\text { Core, } \\
\text { section }\end{array}$} & \multicolumn{2}{|c|}{ Offset $(\mathrm{cm})$} & \multicolumn{2}{|c|}{ Depth CSF-A (m) } & \multirow{2}{*}{$\begin{array}{c}\mathrm{CaCO}_{3} \\
\text { (wt\%) }\end{array}$} & \multirow{2}{*}{$\begin{array}{c} \pm \\
\text { (wt\%) }\end{array}$} & \multirow{2}{*}{$\begin{array}{c}\text { TIC } \\
\text { (wt\%) }\end{array}$} & \multirow{2}{*}{$\begin{array}{c} \pm \\
\text { (wt\%) }\end{array}$} & & & & & & \\
\hline & Top & Bottom & Top & Bottom & & & & & (wt\%) & (wt\%) & (wt\%) & (wt\%) & $(w t \%)$ & (wt\%) \\
\hline $7 \mathrm{H}-6$ & 69 & 70 & 61.29 & 61.30 & 27.9 & & 3.35 & & 0.129 & 0.013 & 0.42 & & 0.008 & \\
\hline $8 \mathrm{H}-5$ & 91 & 92 & 69.52 & 69.53 & 69.1 & & 8.29 & & 0.130 & 0.017 & 0.25 & & 0.015 & \\
\hline $9 \mathrm{H}-2$ & 119 & 120 & 74.81 & 74.82 & 64.1 & & 7.70 & & 0.130 & 0.018 & 0.25 & & 0.017 & \\
\hline $9 \mathrm{H}-7$ & 56 & 57 & 81.64 & 81.65 & 58.9 & & 7.07 & & 0.218 & 0.026 & 0.28 & & 0.016 & \\
\hline $10 \mathrm{H}-1$ & 81 & 82 & 82.41 & 82.42 & 65.8 & & 7.90 & & 0.188 & 0.022 & 0.29 & & 0.024 & \\
\hline $10 \mathrm{H}-5$ & 74 & 75 & 88.30 & 88.31 & 58.4 & & 7.01 & & 0.175 & 0.022 & 0.27 & & 0.020 & \\
\hline $11 \mathrm{H}-1$ & 83 & 84 & 91.93 & 91.94 & 42.9 & & 5.15 & & 0.156 & 0.020 & 0.46 & & 0.021 & \\
\hline $11 \mathrm{H}-7$ & 55 & 56 & 100.49 & 100.50 & 31.2 & & 3.74 & & 0.168 & 0.017 & 0.48 & & 0.021 & \\
\hline $12 \mathrm{H}-1$ & 44 & 45 & 101.04 & 101.05 & 50.4 & & 6.05 & & 0.181 & 0.021 & 0.37 & & 0.023 & \\
\hline $13 \mathrm{H}-1$ & 47 & 48 & 107.77 & 107.78 & 33.9 & & 4.07 & & 0.140 & 0.016 & 0.36 & & 0.029 & \\
\hline $13 \mathrm{H}-5$ & 85 & 86 & 114.15 & 114.16 & 44.9 & & 5.39 & & 0.197 & 0.020 & 0.30 & & 0.029 & \\
\hline $14 \mathrm{H}-1$ & 95 & 96 & 116.85 & 116.86 & 43.0 & & 5.16 & & 0.064 & 0.009 & 0.39 & & 0.027 & \\
\hline $14 \mathrm{H}-6$ & 113 & 114 & 124.54 & 124.55 & 63.6 & & 7.63 & & 0.214 & 0.023 & 0.28 & & 0.042 & \\
\hline $15 \mathrm{H}-2$ & 105 & 106 & 127.95 & 127.96 & 51.8 & & 6.22 & & 0.153 & 0.018 & 0.34 & & 0.051 & \\
\hline $15 \mathrm{H}-6$ & 33 & 34 & 133.25 & 133.26 & 40.0 & & 4.80 & & 0.107 & 0.014 & 0.45 & & 0.035 & \\
\hline 340-U1399 & & & & & & & & & & & & & & \\
\hline $1 \mathrm{H}-1$ & 73 & 74 & 0.73 & 0.74 & 30.8 & & 3.70 & & 0.279 & 0.051 & 0.66 & & 0.025 & \\
\hline $1 \mathrm{H}-2$ & 9 & 10 & 1.59 & 1.60 & 2.6 & & 0.31 & & 0.033 & 0.006 & 0.46 & & 0.006 & \\
\hline $2 \mathrm{H}-4$ & 112 & 113 & 10.72 & 10.73 & 20.9 & & 2.51 & & 0.420 & 0.062 & 0.77 & & 0.054 & \\
\hline $2 \mathrm{H}-6$ & 29 & 30 & 12.89 & 12.90 & 33.2 & & 3.98 & & 0.286 & 0.036 & 0.64 & & 0.047 & \\
\hline $3 \mathrm{H}-1$ & 69 & 70 & 15.29 & 15.30 & 4.5 & & 0.54 & & 0.026 & 0.006 & 0.41 & & 0.009 & \\
\hline $3 \mathrm{H}-3$ & 46 & 47 & 18.06 & 18.07 & 30.0 & & 3.60 & & 0.229 & 0.047 & 0.78 & & 0.076 & \\
\hline $4 \mathrm{H}-2$ & 85 & 86 & 26.45 & 26.46 & 7.8 & & 0.94 & & 0.152 & 0.027 & 0.65 & 0.03 & 0.030 & 0.005 \\
\hline $4 \mathrm{H}-4$ & 105 & 106 & 29.65 & 29.66 & 20.9 & & 2.50 & & 0.293 & 0.056 & 1.20 & 0.04 & 0.088 & 0.000 \\
\hline $5 \mathrm{H}-3$ & 10 & 11 & 36.70 & 36.71 & 22.6 & & 2.71 & & 0.460 & 0.068 & 0.94 & & 0.099 & \\
\hline $5 \mathrm{H}-5$ & 133 & 134 & 40.94 & 40.95 & 9.3 & & 1.12 & & 0.504 & 0.074 & 1.66 & & 0.268 & \\
\hline $6 \mathrm{H}-1$ & 69 & 70 & 43.79 & 43.80 & 26.6 & & 3.19 & & 0.318 & 0.060 & 0.75 & 0.03 & 0.086 & 0.002 \\
\hline $6 \mathrm{H}-6$ & 123 & 124 & 51.63 & 51.64 & 17.4 & & 2.09 & & 0.390 & 0.057 & 1.06 & & 0.070 & \\
\hline $7 \mathrm{H}-1$ & 101 & 102 & 53.61 & 53.62 & 34.7 & & 4.16 & & 0.458 & 0.063 & 0.61 & & 0.072 & \\
\hline 7H-5 & 114 & 115 & 59.74 & 59.75 & 17.5 & 0.1 & 2.11 & 0.01 & 0.279 & 0.041 & 0.82 & & 0.047 & \\
\hline $8 \mathrm{H}-1$ & 44 & 45 & 62.54 & 62.55 & 27.2 & & 3.26 & & 0.297 & 0.043 & 0.79 & & 0.068 & \\
\hline $9 \mathrm{H}-4$ & 129 & 130 & 76.61 & 76.62 & 14.7 & & 1.76 & & 0.322 & 0.051 & 1.03 & & 0.057 & \\
\hline $9 \mathrm{H}-7$ & 14 & 15 & 79.96 & 79.97 & 11.1 & & 1.33 & & 0.582 & 0.080 & 1.16 & & 0.047 & \\
\hline $10 \mathrm{H}-2$ & 102 & 103 & 82.82 & 82.83 & 14.5 & & 1.75 & & 0.329 & 0.055 & 1.40 & & 0.093 & \\
\hline $10 \mathrm{H}-5$ & 60 & 61 & 86.85 & 86.86 & 3.7 & & 0.45 & & 0.614 & 0.080 & 1.40 & 0.01 & 0.053 & 0.002 \\
\hline $11 \mathrm{H}-1$ & 43 & 44 & 90.23 & 90.24 & 7.6 & 0.0 & 0.91 & 0.01 & 0.058 & 0.010 & 0.67 & & 0.026 & \\
\hline $12 \mathrm{H}-3$ & 36 & 37 & 94.29 & 94.30 & 2.2 & & 0.26 & & 0.025 & 0.006 & 0.64 & & 0.010 & \\
\hline $13 \mathrm{H}-1$ & 60 & 61 & 95.70 & 95.71 & 24.2 & 0.2 & 2.91 & 0.02 & 0.216 & 0.039 & 0.70 & & 0.063 & \\
\hline $14 \mathrm{H}-1$ & 52 & 53 & 103.42 & 103.43 & 26.6 & 2.0 & 3.20 & 0.24 & 0.461 & 0.064 & 0.76 & & 0.071 & \\
\hline $14 \mathrm{H}-2$ & 59 & 60 & 104.99 & 105.00 & 8.3 & & 1.00 & & 0.042 & 0.007 & 0.42 & & 0.013 & \\
\hline $15 \mathrm{H}-2$ & 59 & 60 & 114.09 & 114.10 & 35.6 & 0.0 & 4.27 & 0.00 & 0.471 & 0.069 & 0.49 & & 0.110 & \\
\hline $16 \mathrm{H}-4$ & 45 & 46 & 126.40 & 126.41 & 14.3 & & 1.71 & & 0.266 & 0.036 & 0.68 & & 0.117 & \\
\hline $17 \mathrm{H}-3$ & 95 & 96 & 134.91 & 134.92 & 11.1 & 0.1 & 1.33 & 0.00 & 0.141 & 0.019 & 0.65 & & 0.053 & \\
\hline $17 \mathrm{H}-7$ & 12 & 13 & 140.02 & 140.03 & 24.1 & & 2.89 & & 0.384 & 0.055 & 0.64 & 0.01 & 0.083 & 0.000 \\
\hline $18 \mathrm{H}-1$ & 55 & 56 & 141.05 & 141.06 & 20.1 & & 2.41 & & 0.308 & 0.049 & 0.81 & & 0.150 & \\
\hline $20 \mathrm{H}-1$ & 100 & 101 & 154.40 & 154.41 & 14.2 & & 1.70 & & 0.322 & 0.050 & 0.84 & & 0.049 & \\
\hline $20 \mathrm{H}-4$ & 39 & 40 & 158.29 & 158.30 & 21.1 & & 2.54 & & 0.317 & 0.041 & 0.91 & & 0.170 & \\
\hline $23 \mathrm{H}-1$ & 62 & 63 & 167.82 & 167.83 & 20.6 & 0.2 & 2.48 & 0.02 & 0.239 & 0.031 & 0.92 & & 0.073 & \\
\hline $23 \mathrm{H}-5$ & 129 & 130 & 174.45 & 174.46 & 23.1 & 0.3 & 2.77 & 0.03 & 0.274 & 0.045 & 0.75 & & 0.052 & \\
\hline $24 \mathrm{H}-1$ & 38 & 39 & 175.78 & 175.79 & 17.4 & & 2.08 & & 0.339 & 0.048 & 1.01 & & 0.064 & \\
\hline $25 \mathrm{H}-1$ & 70 & 71 & 185.20 & 185.21 & 21.8 & & 2.62 & & 0.338 & 0.056 & 0.88 & 0.01 & 0.049 & 0.002 \\
\hline $26 \mathrm{H}-1$ & 15 & 16 & 191.85 & 191.86 & 33.2 & & 3.99 & & 0.256 & 0.050 & 0.56 & & 0.075 & \\
\hline $26 \mathrm{H}-3$ & 80 & 81 & 195.54 & 195.55 & 2.1 & & 0.25 & & 0.055 & 0.015 & 0.85 & & 0.008 & \\
\hline $27 \mathrm{H}-1$ & 78 & 79 & 198.48 & 198.49 & 20.6 & & 2.47 & & 0.353 & 0.058 & 1.06 & 0.15 & 0.048 & 0.004 \\
\hline $27 \mathrm{H}-4$ & 32 & 33 & 202.56 & 202.57 & 26.1 & & 3.13 & & 0.368 & 0.053 & 0.64 & & 0.060 & \\
\hline $28 \mathrm{H}-2$ & 18 & 19 & 205.06 & 205.07 & 17.8 & & 2.13 & & 0.271 & 0.045 & 0.94 & & 0.039 & \\
\hline $29 \mathrm{H}-1$ & 72 & 73 & 206.22 & 206.23 & 29.5 & & 3.54 & & 0.199 & 0.036 & 0.77 & & 0.069 & \\
\hline $31 X-1$ & 77 & 78 & 217.87 & 217.88 & 30.8 & & 3.70 & & 0.170 & 0.047 & 0.65 & & 0.086 & \\
\hline $33 X-1$ & 95 & 96 & 237.25 & 237.26 & 25.5 & & 3.06 & & 0.329 & 0.045 & 0.74 & & 0.047 & \\
\hline $34 \mathrm{X}-\mathrm{CC}$ & 20 & 21 & 246.83 & 246.84 & 12.1 & & 1.46 & & 0.076 & 0.009 & 0.61 & & 0.026 & \\
\hline $35 \mathrm{X}-\mathrm{CC}$ & 16 & 17 & 256.25 & 256.26 & 16.1 & & 1.93 & & 0.068 & 0.010 & 0.55 & & 0.019 & \\
\hline $36 X-2$ & 57 & 58 & 267.17 & 267.18 & 21.4 & & 2.56 & & 0.087 & 0.016 & 0.47 & & 0.040 & \\
\hline $36 X-3$ & 98 & 99 & 269.08 & 269.09 & 22.3 & 0.0 & 2.68 & 0.01 & 0.457 & 0.072 & 0.75 & & 0.099 & \\
\hline 340-U1400 & & & & & & & & & & & & & & \\
\hline $1 \mathrm{H}-1$ & 19 & 20 & 0.19 & 0.20 & 18.6 & & 2.23 & & 0.400 & 0.052 & 0.88 & & 0.023 & \\
\hline $1 \mathrm{H}-2$ & 105 & 106 & 2.55 & 2.56 & 9.8 & & & & 0.162 & 0.022 & 0.92 & & 0.024 & \\
\hline $7 \mathrm{H}-3$ & 51 & 52 & 28.85 & 28.86 & 34.6 & & & & 0.327 & 0.040 & 0.60 & & 0.054 & \\
\hline $7 \mathrm{H}-6$ & 65 & 66 & 33.49 & 33.50 & 24.5 & 0.1 & 2.94 & 0.01 & 0.270 & 0.027 & 0.60 & & 0.037 & \\
\hline
\end{tabular}


Table T4 (continued).

\begin{tabular}{|c|c|c|c|c|c|c|c|c|c|c|c|c|c|c|}
\hline \multirow{2}{*}{$\begin{array}{l}\text { Core, } \\
\text { section }\end{array}$} & \multicolumn{2}{|c|}{ Offset $(\mathrm{cm})$} & \multicolumn{2}{|c|}{ Depth CSF-A (m) } & \multirow{2}{*}{$\begin{array}{c}\mathrm{CaCO}_{3} \\
\text { (wt\%) }\end{array}$} & \multirow{2}{*}{$\begin{array}{c} \pm \\
(w t \%)\end{array}$} & \multirow{2}{*}{$\begin{array}{c}\text { TIC } \\
(w t \%)\end{array}$} & \multirow{2}{*}{$\begin{array}{c} \pm \\
\text { (wt\%) }\end{array}$} & \multirow{2}{*}{$\begin{array}{c}\mathrm{OC} \\
(w t \%)\end{array}$} & \multirow{2}{*}{$\underset{(w t \%)}{N}$} & \multirow{2}{*}{$\begin{array}{c}\mathrm{Fe}_{\mathrm{R}} \\
\text { (wt\%) }\end{array}$} & \multirow{2}{*}{$\begin{array}{c} \pm \\
(w t \%)\end{array}$} & \multirow{2}{*}{$\begin{array}{c}\mathrm{Mn}_{\mathrm{R}} \\
\text { (wt\%) }\end{array}$} & \multirow{2}{*}{$\begin{array}{c} \pm \\
\text { (wt\%) }\end{array}$} \\
\hline & Top & Bottom & Top & Bottom & & & & & & & & & & \\
\hline $10 \mathrm{H}-1$ & 88 & 89 & 49.38 & 49.39 & 23.5 & & 2.82 & & 0.309 & 0.047 & 0.89 & & 0.089 & \\
\hline $10 \mathrm{H}-5$ & 52 & 53 & 55.03 & 55.04 & 22.8 & 0.3 & 2.71 & & 0.247 & 0.040 & 0.78 & & 0.145 & \\
\hline $11 \mathrm{H}-2$ & 103 & 104 & 60.53 & 60.54 & 11.6 & & 1.39 & & 0.270 & 0.040 & 1.20 & 0.03 & 0.049 & 0.001 \\
\hline $11 \mathrm{H}-7$ & 30 & 31 & 66.92 & 66.93 & 17.6 & 0.1 & 2.12 & 0.01 & 0.133 & 0.019 & 0.86 & & 0.069 & \\
\hline $15 \mathrm{H}-2$ & 93 & 94 & 95.39 & 95.40 & 22.1 & & 2.65 & & 0.270 & 0.044 & 0.81 & & 0.086 & \\
\hline $15 \mathrm{H}-5$ & 63 & 64 & 99.53 & 99.54 & 23.4 & 0.5 & 2.81 & 0.06 & 0.294 & 0.044 & 0.87 & & 0.094 & \\
\hline $17 \mathrm{H}-1$ & 123 & 124 & 106.93 & 106.94 & 19.9 & & 2.39 & & 0.291 & 0.040 & 0.81 & & 0.065 & \\
\hline $17 \mathrm{H}-5$ & 80 & 81 & 112.43 & 112.44 & 4.9 & & 0.59 & & 0.099 & 0.014 & 1.23 & & 0.021 & \\
\hline $18 \mathrm{H}-3$ & 53 & 54 & 118.67 & 118.68 & 6.9 & & 0.82 & & 0.207 & 0.020 & 1.09 & & 0.035 & \\
\hline $18 \mathrm{H}-7$ & 57 & 58 & 124.59 & 124.60 & 15.7 & & 1.88 & & 0.235 & 0.029 & 0.81 & & 0.053 & \\
\hline $19 \mathrm{H}-3$ & 60 & 61 & 128.22 & 128.23 & 14.0 & & 1.68 & & 0.248 & 0.036 & 1.07 & & 0.060 & \\
\hline $20 \mathrm{H}-4$ & 108 & 109 & 134.39 & 134.40 & 12.6 & & 1.51 & & 0.152 & 0.020 & 0.97 & & 0.046 & \\
\hline $21 \mathrm{H}-1$ & 110 & 111 & 137.70 & 137.71 & 20.7 & & 2.48 & & 0.239 & 0.043 & 1.07 & & 0.177 & \\
\hline $21 \mathrm{H}-5$ & 34 & 35 & 142.79 & 142.80 & 13.7 & & 1.65 & & 0.263 & 0.022 & 0.74 & 0.04 & 0.049 & 0.002 \\
\hline $22 \mathrm{H}-1$ & 115 & 116 & 147.25 & 147.26 & 13.5 & 0.4 & 1.62 & 0.05 & 0.142 & 0.027 & 0.68 & & 0.062 & \\
\hline $22 \mathrm{H}-7$ & 32 & 33 & 155.03 & 155.04 & 19.7 & 0.9 & 2.37 & 0.10 & 0.265 & 0.044 & 0.62 & & 0.101 & \\
\hline $23 \mathrm{H}-2$ & 90 & 91 & 157.96 & 157.97 & 24.6 & & 2.95 & & 0.153 & 0.030 & 0.53 & 0.02 & 0.086 & 0.001 \\
\hline $23 \mathrm{H}-7$ & 13 & 14 & 164.08 & 164.09 & 20.2 & 0.0 & 2.43 & 0.00 & 0.218 & 0.043 & 0.79 & 0.00 & 0.073 & 0.001 \\
\hline $24 \mathrm{H}-2$ & 40 & 41 & 166.97 & 166.98 & 32.4 & 0.5 & 3.89 & 0.05 & 0.242 & 0.037 & 0.64 & & 0.108 & \\
\hline $24 \mathrm{H}-6$ & 67 & 69 & 173.12 & 173.14 & 25.0 & & 3.00 & & 0.209 & 0.038 & 0.68 & & 0.106 & \\
\hline $25 \mathrm{H}-2$ & 58 & 59 & 176.64 & 176.65 & 34.3 & & 4.12 & & 0.186 & 0.039 & 0.51 & & 0.149 & \\
\hline $25 \mathrm{H}-6$ & 28 & 29 & 182.20 & 182.21 & 27.4 & & 3.29 & & 0.206 & 0.040 & 0.62 & & 0.126 & \\
\hline $26 \mathrm{H}-2$ & 48 & 49 & 185.95 & 185.96 & 8.2 & & 0.98 & & 0.151 & 0.017 & 0.76 & & 0.026 & \\
\hline $26 \mathrm{H}-7$ & 75 & 76 & 193.58 & 193.59 & 7.7 & & 0.92 & & 0.136 & 0.017 & 0.78 & & 0.050 & \\
\hline $27 \mathrm{H}-1$ & 62 & 63 & 194.12 & 194.13 & 4.4 & 0.2 & 0.53 & 0.02 & 0.177 & 0.019 & 1.42 & & 0.022 & \\
\hline $28 \mathrm{H}-2$ & 53 & 54 & 204.99 & 205.00 & 12.6 & 0.1 & 1.51 & 0.01 & 0.258 & 0.031 & 0.68 & 0.00 & 0.062 & 0.001 \\
\hline $28 \mathrm{H}-6$ & 33 & 34 & 210.65 & 210.66 & 2.1 & 0.1 & 0.25 & 0.01 & 0.096 & 0.010 & 1.06 & & 0.017 & \\
\hline 340-U1400 & & & & & & & & & & & & & & \\
\hline $10 \mathrm{H}-1$ & 60 & 61 & 79.30 & 79.31 & 8.3 & & 1.00 & & 0.158 & 0.024 & 0.78 & & 0.029 & \\
\hline $11 \mathrm{H}-1$ & 116 & 117 & 85.96 & 85.97 & 18.6 & 0.4 & 2.23 & 0.05 & 0.225 & 0.032 & 0.72 & & 0.078 & \\
\hline $12 \mathrm{H}-2$ & 25 & 26 & 95.55 & 95.56 & 14.1 & 0.3 & 1.69 & 0.03 & 0.218 & 0.029 & 0.73 & & 0.040 & \\
\hline $13 \mathrm{H}-6$ & 7 & 8 & 110.90 & 110.91 & 16.4 & & 1.96 & & 0.301 & 0.042 & 0.57 & & 0.041 & \\
\hline $22 \mathrm{H}-4$ & 43 & 44 & 186.42 & 186.43 & 10.0 & & 1.20 & & 0.097 & 0.018 & 0.54 & & 0.015 & \\
\hline $27 X-2$ & 96 & 97 & 227.76 & 227.77 & 7.9 & & 0.95 & & 0.240 & 0.027 & 0.93 & & 0.039 & \\
\hline $28 X-1$ & 52 & 53 & 235.42 & 235.43 & 6.7 & & 0.81 & & 0.141 & 0.014 & 0.86 & & 0.105 & \\
\hline $29 X-2$ & 50 & 51 & 246.50 & 246.51 & 18.8 & & 2.26 & & 0.336 & 0.041 & 0.72 & & 0.027 & \\
\hline $30 X-1$ & 31 & 32 & 254.41 & 254.42 & 23.6 & 0.5 & 2.85 & 0.08 & 0.258 & 0.034 & 0.61 & & 0.091 & \\
\hline $31 X-1$ & 21 & 22 & 263.91 & 263.92 & 13.0 & & 1.56 & & 0.147 & 0.022 & 0.79 & & 0.042 & \\
\hline $32 X-3$ & 122 & 123 & 277.52 & 277.53 & 27.7 & & 3.32 & & 0.331 & 0.035 & 0.89 & & 0.102 & \\
\hline $33 X-3$ & 33 & 34 & 286.23 & 286.24 & 25.6 & & 3.07 & & 0.340 & 0.036 & 0.79 & & 0.076 & \\
\hline $35 X-3$ & 49 & 50 & 295.99 & 296.00 & 18.6 & & 2.23 & & 0.398 & 0.035 & 1.04 & 0.01 & 0.054 & 0.002 \\
\hline $36 X-2$ & 113 & 114 & 304.73 & 304.74 & 23.4 & & 2.81 & & 0.185 & 0.028 & 0.79 & & 0.068 & \\
\hline $37 X-2$ & 21 & 22 & 313.43 & 313.44 & 21.5 & 0.3 & 2.58 & 0.04 & 0.347 & 0.048 & 0.89 & 0.06 & 0.072 & 0.001 \\
\hline $38 X-1$ & 111 & 112 & 322.01 & 322.02 & 31.4 & & 3.77 & & 0.352 & 0.045 & 0.74 & & 0.089 & \\
\hline $38 X-6$ & 98 & 99 & 329.38 & 329.39 & 7.6 & & 0.91 & & 0.137 & 0.014 & 0.83 & & 0.031 & \\
\hline $39 X-2$ & 77 & 78 & 332.77 & 332.78 & 24.0 & & 2.87 & & 0.216 & 0.030 & 0.61 & & 0.140 & \\
\hline $40 X-1$ & 94 & 95 & 341.04 & 341.05 & 9.3 & & 1.11 & & 0.140 & 0.016 & 0.75 & & 0.032 & \\
\hline $41 X-1$ & 115 & 116 & 350.85 & 350.86 & 25.0 & & 3.00 & & 0.265 & 0.039 & 0.61 & & 0.082 & \\
\hline $42 X-2$ & 66 & 67 & 361.46 & 361.47 & 9.8 & & 1.17 & & 0.099 & 0.013 & 0.81 & & 0.028 & \\
\hline $43 X-1$ & 46 & 47 & 369.36 & 369.37 & 10.8 & 1.0 & 1.30 & 0.12 & 0.106 & 0.012 & 0.89 & 0.01 & 0.056 & 0.000 \\
\hline $44 X-2$ & 72 & 73 & 380.57 & 380.58 & 17.4 & & 2.09 & & 0.195 & 0.026 & 0.72 & & 0.046 & \\
\hline $45 X-4$ & 40 & 41 & 392.95 & 392.96 & 15.3 & & 1.84 & & 0.207 & 0.028 & 0.82 & & 0.062 & \\
\hline $46 X-1$ & 35 & 36 & 397.95 & 397.96 & 16.1 & 0.2 & 1.93 & 0.03 & 0.336 & 0.037 & 0.76 & 0.01 & 0.057 & 0.000 \\
\hline $47 X-1$ & 65 & 66 & 407.85 & 407.86 & 22.0 & & 2.64 & & 0.212 & 0.032 & 0.54 & & 0.081 & \\
\hline $48 \mathrm{X}-\mathrm{CC}$ & 13 & 14 & 417.46 & 417.47 & 34.0 & & 4.08 & & 0.312 & 0.041 & 0.41 & & 0.136 & \\
\hline $49 X-1$ & 61 & 62 & 427.01 & 427.02 & 25.7 & 1.1 & 3.08 & 0.13 & 0.310 & 0.041 & 0.51 & & 0.114 & \\
\hline
\end{tabular}

$\mathrm{TIC}=$ total inorganic carbon, $\mathrm{OC}=$ organic carbon. $\mathrm{Fe}_{\mathrm{R}}=$ dithionite-extractable iron, $\mathrm{Mn}_{\mathrm{R}}=$ dithionite-extractable manganese . 
Table T5. Composition of solid phases in high-resolution sections, Sites U1399 and U1400. (Continued on next page.)

\begin{tabular}{|c|c|c|c|c|c|c|c|c|c|c|c|c|c|c|c|c|c|}
\hline \multirow{2}{*}{$\begin{array}{l}\text { Core, } \\
\text { section }\end{array}$} & \multicolumn{2}{|c|}{ Offset $(\mathrm{cm})$} & \multicolumn{2}{|c|}{ Depth CSF-A (m) } & \multirow{2}{*}{$\begin{array}{l}\mathrm{CaCO}_{3} \\
\text { (wt\%) }\end{array}$} & \multirow{2}{*}{$\begin{array}{c} \pm \\
(w t \%)\end{array}$} & \multirow{2}{*}{$\begin{array}{c}\mathrm{TIC} \\
(w t \%)\end{array}$} & \multirow{2}{*}{$\begin{array}{c} \pm \\
(w+\%)\end{array}$} & \multirow{2}{*}{$\begin{array}{l}\mathrm{OC} \\
(\mathrm{wt} \%)\end{array}$} & \multirow{2}{*}{$\begin{array}{c} \pm \\
(w+\%)\end{array}$} & \multirow{2}{*}{$\underset{(w t \%)}{N}$} & \multirow{2}{*}{$\begin{array}{l} \pm \\
(w+\%)\end{array}$} & \multirow{2}{*}{$\begin{array}{c}\mathrm{Fe}_{\mathrm{R}} \\
(\mathrm{wt} \%)\end{array}$} & \multirow{2}{*}{$\begin{array}{c} \pm \\
(w+\%)\end{array}$} & \multirow{2}{*}{$\begin{array}{c}\mathrm{Mn}_{\mathrm{R}} \\
(\mathrm{wt} \%)\end{array}$} & \multirow{2}{*}{$\begin{array}{c} \pm \\
(w t \%)\end{array}$} & \multirow[b]{2}{*}{ Lithology descriptions } \\
\hline & Top & Bottom & Top & Bottom & & & & & & & & & & & & & \\
\hline $340-U 1399$ & & & & & & & & & & & & & & & & & \\
\hline $3 \mathrm{H}-3$ & 88 & 90 & 19.08 & 19.10 & 9.2 & & 1.10 & & 0.206 & & 0.031 & & 1.13 & & 0.06 & & Mud with hints of silt/very fine grained sand \\
\hline & 93 & 95 & 19.13 & 19.15 & 5.7 & 0.1 & 0.68 & 0.01 & 0.143 & & 0.019 & & 1.25 & 0.00 & 0.05 & 0.00 & Very fine sand/silt with some mud present \\
\hline & 98 & 100 & 19.18 & 19.20 & 11.7 & & 1.41 & & 0.241 & & 0.032 & & 0.80 & 0.04 & 0.06 & 0.00 & Very fine sand/silt with some mud present \\
\hline & 101 & 103 & 19.21 & 19.23 & 7.2 & 0.0 & 0.86 & 0.00 & 0.072 & & 0.009 & & 0.56 & & 0.03 & & Fine-grained sand \\
\hline & 104 & 106 & 19.24 & 19.26 & 41.4 & 0.1 & 4.96 & 0.01 & 0.288 & & 0.040 & & 0.59 & & 0.07 & & $\begin{array}{l}35 \%-40 \% \text { shell fragments, } 60 \%-65 \% \text { fine-grained } \\
\text { sand/silt }\end{array}$ \\
\hline & 108 & 110 & 19.28 & 19.30 & 16.8 & 0.3 & 2.01 & 0.03 & 0.283 & & 0.049 & & 1.11 & & 0.13 & & Mud \\
\hline $7 \mathrm{H}-3$ & 17 & 19 & 56.37 & 56.39 & 13.4 & 0.5 & 1.61 & 0.06 & 0.273 & & 0.039 & & 0.90 & & 0.09 & & Mud with hint of silt \\
\hline & 21 & 23 & 56.41 & 56.43 & 11.4 & & 1.37 & & 0.260 & & 0.037 & & 1.15 & & 0.08 & & Mud \\
\hline & 25 & 27 & 56.45 & 56.47 & 15.6 & 0.2 & 1.88 & 0.03 & 0.265 & & 0.046 & & 1.09 & & 0.11 & & Mud with silt \\
\hline & 30 & 32 & 56.50 & 56.52 & 17.6 & & 2.12 & & 0.215 & & 0.041 & & 0.94 & 0.00 & 0.10 & 0.00 & Mud with hints of silt \\
\hline & 34 & 36 & 56.54 & 56.56 & 21.5 & 0.1 & 2.58 & 0.02 & 0.261 & & 0.048 & & 0.82 & & 0.11 & & Mud with hints of silt \\
\hline & 65 & 67 & 56.85 & 56.87 & 20.3 & & 2.44 & & 0.203 & & 0.032 & & 0.75 & & 0.14 & & Mud with hints of silt \\
\hline & 68 & 70 & 56.88 & 56.90 & 22.9 & & 2.75 & & 0.272 & & 0.038 & & 0.82 & 0.02 & 0.15 & 0.00 & $2 \%$ very fine grained sand, mud \\
\hline & 73 & 75 & 56.93 & 56.95 & 17.5 & 0.6 & 2.10 & 0.07 & 0.144 & & 0.025 & & 0.78 & & 0.08 & & Fine-very fine grained sand $50 \%-75 \%, 25 \%-50 \%$ mud \\
\hline & 76 & 78 & 56.96 & 56.98 & 17.3 & 0.1 & 2.07 & 0.01 & 0.208 & 0.010 & 0.041 & 0.003 & 0.93 & & 0.10 & & Mud \\
\hline & 81 & 83 & 57.01 & 57.03 & 13.5 & 0.1 & 1.63 & 0.01 & 0.179 & & 0.033 & & 1.21 & 0.01 & 0.10 & 0.00 & Silt/very fine grained sand dominates, clay is present \\
\hline $11 \mathrm{H}-2$ & 79 & 81 & 92.09 & 92.11 & 5.9 & 0.1 & 0.70 & 0.02 & 0.083 & & 0.013 & & 0.46 & & 0.03 & & $<1 \%$ sand, $25 \%-30 \%$ tephra, $70 \%-74 \%$ mud $/$ silt \\
\hline & 81 & 83 & 92.11 & 92.13 & 21.1 & 0.9 & 2.54 & 0.10 & 0.253 & & 0.031 & & 0.70 & & 0.07 & & $<1 \%$ sand, $5 \%-10 \%$ tephra, $89 \%-94 \%$ mud \\
\hline & 83 & 85 & 92.13 & 92.15 & 10.8 & & 1.30 & & 0.147 & & 0.019 & & 0.61 & & 0.05 & & $<1 \%$ sand, $10 \%$ tephra, $89 \%$ silty $/$ mud \\
\hline & 87 & 89 & 92.17 & 92.19 & 17.1 & 0.1 & 2.05 & 0.01 & 0.179 & & 0.025 & & 0.85 & 0.03 & 0.09 & 0.00 & Mud with hints of silt \\
\hline & 92 & 94 & 92.22 & 92.24 & 16.8 & & 2.02 & & 0.165 & & 0.025 & & 0.81 & 0.02 & 0.09 & 0.00 & $2 \%$ sand, $<1 \%$ tephra, $97 \%$ mud with hints of silt \\
\hline & 96 & 98 & 92.26 & 92.28 & 4.3 & 0.2 & 0.52 & 0.02 & 0.121 & & 0.009 & & 0.46 & & 0.02 & & $10 \%-15 \%$ tephra, silt/fine sand, minimal mud \\
\hline $16 \mathrm{H}-2$ & 24 & 26 & 125.94 & 125.96 & 11.5 & & 1.39 & & 0.249 & & 0.032 & & 0.93 & 0.02 & 0.08 & 0.00 & Mud, $<1 \%$ medium-grained sand \\
\hline & 28 & 30 & 125.98 & 126.00 & 6.2 & 0.0 & 0.74 & 0.00 & 0.184 & & 0.025 & & 1.14 & 0.13 & 0.07 & 0.00 & $<1 \%$ medium sand, mud with hints of silt \\
\hline & 32 & 34 & 126.02 & 126.04 & 8.4 & 0.1 & 1.01 & 0.01 & 0.180 & & 0.023 & & 1.05 & & 0.07 & & Muddy sand, sand is very fine to fine grained \\
\hline & 36 & 38 & 126.06 & 126.08 & 15.5 & 0.3 & 1.86 & 0.04 & 0.310 & & 0.045 & & 1.15 & & 0.26 & & Mud with hints of silt \\
\hline & 41 & 43 & 126.11 & 126.13 & 10.4 & & 1.25 & & 0.219 & & 0.032 & & 1.12 & & 0.14 & & Mud with minor amounts of silt \\
\hline $19 \mathrm{H}-2$ & 49 & 51 & 135.59 & 135.61 & 22.4 & & 2.69 & & 0.297 & & 0.054 & & 0.83 & 0.07 & 0.14 & 0.00 & Mud with hints of silt \\
\hline & 52 & 54 & 135.62 & 135.64 & 20.4 & 0.2 & 2.45 & 0.03 & 0.337 & 0.009 & 0.052 & 0.002 & 0.84 & & 0.10 & & Mud with hints of silt \\
\hline & 57 & 59 & 135.67 & 135.69 & 30.2 & 0.5 & 3.62 & 0.07 & 0.174 & & 0.023 & & 0.52 & & 0.08 & & Fine sand with hints of mud \\
\hline & 62 & 64 & 135.72 & 135.74 & 26.5 & 0.2 & 3.18 & 0.03 & 0.137 & & 0.019 & & 0.55 & & 0.07 & & Very fine to fine-grained sand with hints of mud \\
\hline & 68 & 70 & 135.78 & 135.80 & 26.3 & 0.6 & 3.15 & 0.08 & 0.140 & & 0.019 & & 0.59 & & 0.08 & & Silty/sand with minimal mud \\
\hline & 71 & 73 & 135.81 & 135.83 & 18.6 & 0.1 & 2.23 & 0.01 & 0.243 & & 0.044 & & 0.83 & 0.02 & 0.15 & 0.01 & Mud \\
\hline $25 \mathrm{H}-2$ & 94 & 96 & 168.84 & 168.86 & 16.0 & 0.1 & 1.92 & 0.01 & 0.175 & & 0.027 & & 0.93 & & 0.14 & & Mud \\
\hline & 98 & 100 & 168.88 & 168.90 & 15.5 & 0.0 & 1.86 & 0.00 & 0.103 & & 0.022 & & 0.54 & 0.01 & 0.17 & 0.01 & Mud with hints of silt \\
\hline & 103 & 105 & 168.93 & 168.95 & 3.9 & 0.1 & 0.47 & 0.01 & 0.068 & & 0.014 & & 0.37 & & 0.10 & & Very fine sand to silt of weathered tephra \\
\hline & 109 & 111 & 168.99 & 169.01 & 0.1 & 0.1 & 0.01 & 0.01 & 0.046 & 0.008 & 0.008 & 0.000 & 0.23 & & 0.00 & & Very fine sand to silt of weathered tephra \\
\hline & 114 & 116 & 169.04 & 169.06 & 21.5 & 0.0 & 2.58 & 0.00 & 0.209 & 0.005 & 0.050 & 0.003 & 0.87 & & 0.18 & & Mud with hints of silt \\
\hline $340-U 1400$ & & & & & & & & & & & & & & & & & \\
\hline $16 \mathrm{H}-2$ & 33 & 34 & 104.17 & 104.18 & 1.0 & 0.0 & 0.12 & 0.01 & 0.141 & & 0.006 & & 0.27 & & 0.01 & & Medium/coarse sand \\
\hline & 37 & 38 & 104.21 & 104.22 & 0.8 & 0.3 & 0.10 & 0.03 & 0.021 & 0.003 & 0.006 & 0.001 & 0.32 & & 0.01 & & Medium/fine sand with tephra present \\
\hline & 42 & 43 & 104.26 & 104.27 & 1.2 & 0.1 & 0.15 & 0.01 & 0.062 & 0.002 & 0.008 & 0.002 & 0.74 & & 0.02 & & Mud with $<5 \%$ medium sand \\
\hline & 46 & 47 & 104.30 & 104.31 & 6.5 & 0.1 & 0.78 & 0.02 & 0.098 & & 0.014 & & 0.59 & & 0.04 & & Mud with $<2 \%$ fine sand \\
\hline & 50 & 51 & 104.34 & 104.35 & 3.8 & 0.1 & 0.46 & 0.01 & 0.080 & 0.004 & 0.009 & 0.001 & 0.84 & 0.01 & 0.04 & 0.00 & Mud with $2 \%$ fine/medium sand \\
\hline & 55 & 56 & 104.39 & 104.40 & 0.5 & 0.2 & 0.06 & 0.02 & 0.019 & 0.007 & 0.005 & 0.003 & 0.34 & & 0.01 & & Medium-grained sand \\
\hline
\end{tabular}


Table T5. (continued).

\begin{tabular}{|c|c|c|c|c|c|c|c|c|c|c|c|c|c|c|c|c|c|}
\hline \multirow{2}{*}{$\begin{array}{c}\text { Core, } \\
\text { section }\end{array}$} & \multicolumn{2}{|c|}{ Offset $(\mathrm{cm})$} & \multicolumn{2}{|c|}{ Depth CSF-A (m) } & \multirow{2}{*}{$\begin{array}{l}\mathrm{CaCO}_{3} \\
\text { (wt\%) }\end{array}$} & \multirow{2}{*}{$\begin{array}{c} \pm \\
\text { (wt\%) }\end{array}$} & \multirow{2}{*}{$\begin{array}{c}\text { TIC } \\
\text { (wt\%) }\end{array}$} & \multirow{2}{*}{$\begin{array}{c} \pm \\
(w t \%)\end{array}$} & \multirow{2}{*}{$\begin{array}{c}\mathrm{OC} \\
\text { (wt\%) }\end{array}$} & \multirow{2}{*}{$\begin{array}{c} \pm \\
\text { (wt\%) }\end{array}$} & \multirow{2}{*}{$\underset{(w t \%)}{\mathrm{N}}$} & \multirow{2}{*}{$\begin{array}{c} \pm \\
(w t \%)\end{array}$} & \multirow{2}{*}{$\begin{array}{c}\mathrm{Fe}_{\mathrm{R}} \\
(\mathrm{wt} \%)\end{array}$} & \multirow{2}{*}{$\begin{array}{c} \pm \\
(w t \%)\end{array}$} & \multirow{2}{*}{$\begin{array}{c}\mathrm{Mn}_{\mathrm{R}} \\
(\mathrm{wt} \%)\end{array}$} & \multirow{2}{*}{$\stackrel{ \pm}{ \pm}$} & \multirow[b]{2}{*}{ Lithology descriptions } \\
\hline & Top & Bottom & Top & Bottom & & & & & & & & & & & & & \\
\hline \multirow[t]{5}{*}{$26 \mathrm{H}-5$} & 48 & 49 & 190.36 & 190.37 & 17.9 & & 2.15 & & 0.149 & & 0.024 & & 0.84 & & 0.16 & & Mud \\
\hline & 53 & 54 & 190.41 & 190.42 & 14.6 & 0.2 & 1.75 & 0.02 & 0.092 & & 0.015 & & 0.74 & & 0.12 & & $50 \%$ mud, $50 \%$ very fine to fine-grained sand \\
\hline & 57 & 58 & 190.45 & 190.46 & 16.7 & 0.1 & 2.00 & 0.01 & 0.130 & & 0.022 & & 0.80 & 0.00 & 0.15 & 0.00 & Mud \\
\hline & 61 & 62 & 190.49 & 190.50 & 10.1 & 0.3 & 1.21 & 0.03 & 0.128 & & 0.018 & & 1.04 & & 0.10 & & Mud with silt present \\
\hline & 66 & 67 & 190.54 & 190.55 & 2.5 & 0.1 & 0.30 & 0.01 & 0.091 & & 0.012 & & 1.23 & 0.09 & 0.05 & 0.00 & Mud--> volcaniclastic mud according to cruise \\
\hline \multicolumn{18}{|c|}{ 340-U1400C- } \\
\hline \multirow[t]{6}{*}{$48 \mathrm{X}-1$} & 14 & 15 & 416.94 & 416.95 & 1.3 & 0.1 & 0.15 & 0.15 & 0.105 & & 0.014 & & 1.06 & & 0.03 & & Mud/mudstone with some silt \\
\hline & 18 & 19 & 416.98 & 416.99 & 3.2 & 0.2 & 0.38 & 0.03 & 0.074 & & 0.013 & & 0.74 & & 0.05 & & Mud/mudstone with minor silt \\
\hline & 22 & 23 & 417.02 & 417.03 & 2.1 & 0.1 & 0.26 & 0.01 & 0.064 & 0.016 & 0.007 & 0.002 & 0.44 & & 0.03 & & $20 \%-30 \%$ very fine to fine-grained sand, $\mathrm{mud} / \mathrm{silt}$ \\
\hline & 25 & 26 & 417.05 & 417.06 & 2.5 & 0.1 & 0.30 & 0.01 & 0.076 & 0.003 & 0.011 & 0.001 & 0.23 & & 0.04 & & Silty mud \\
\hline & 29 & 30 & 417.09 & 417.10 & 1.6 & 0.1 & 0.19 & 0.01 & 0.046 & 0.003 & 0.006 & 0.002 & 0.42 & 0.02 & 0.02 & 0.00 & $50 \%$ sand, $50 \%$ silty $/$ mud, tephra present? $<1 \%$ \\
\hline & 39 & 40 & 417.19 & 417.20 & 25.0 & 0.2 & 3.01 & 0.02 & 0.241 & & 0.039 & & 0.79 & & 0.48 & & Mud \\
\hline
\end{tabular}

$\mathrm{TIC}=$ total inorganic carbon, $\mathrm{OC}=$ organic carbon. $\mathrm{Fe}_{\mathrm{R}}=$ dithionite-extractable iron, $\mathrm{Mn}_{\mathrm{R}}=$ dithionite-extractable manganese. 
Table T6. Dithionite-extractable metals from Site U1396 ash layers.

\begin{tabular}{|c|c|c|c|c|c|c|c|c|c|c|c|}
\hline \multirow[b]{2}{*}{$\begin{array}{c}\text { Core, } \\
\text { section }\end{array}$} & \multicolumn{2}{|c|}{ Offset $(\mathrm{cm})$} & \multirow{2}{*}{$\begin{array}{c}\mathrm{Fe} \\
\text { (wt\%) }\end{array}$} & \multirow{2}{*}{$\begin{array}{c} \pm \\
\text { (wt\%) }\end{array}$} & \multirow{2}{*}{$\begin{array}{c}\mathrm{Mn} \\
(w t \%)\end{array}$} & \multirow{2}{*}{$\underset{\text { (wt\%) }}{ \pm}$} & \multirow{2}{*}{$\begin{array}{c}\mathrm{Al} \\
\text { (wt\%) }\end{array}$} & \multirow{2}{*}{$\begin{array}{c} \pm \\
\text { (wt\%) }\end{array}$} & \multirow{2}{*}{$\begin{array}{c}\mathrm{OC} \\
\text { (wt } \%)\end{array}$} & \multirow{2}{*}{$\begin{array}{c} \pm \\
\text { (wt\%) }\end{array}$} & \multirow{2}{*}{$\begin{array}{c}\mathrm{TIC} \\
\text { (wt\%) }\end{array}$} \\
\hline & Top & Bottom & & & & & & & & & \\
\hline \multicolumn{12}{|c|}{ 340-U1396C- } \\
\hline $1 \mathrm{H}-2$ & 51 & 52 & 0.39 & & 0.0052 & & 0.030 & & 0.06 & & 3.18 \\
\hline $1 \mathrm{H}-3$ & 79 & 80 & 0.55 & & 0.0080 & & & & 0.14 & & 0.33 \\
\hline $2 \mathrm{H}-1$ & 76 & 77 & 0.43 & & 0.0043 & & 0.025 & & 0.02 & & 0.73 \\
\hline $2 \mathrm{H}-7$ & 64 & 65 & 0.83 & & 0.0056 & & 0.070 & & 0.08 & & 0.39 \\
\hline $3 \mathrm{H}-2$ & 59 & 60 & 0.57 & & 0.0057 & & 0.041 & & 0.04 & & 0.83 \\
\hline $4 \mathrm{H}-6$ & 42 & 43 & 0.39 & & 0.0068 & & 0.057 & & 0.09 & & 2.10 \\
\hline $6 \mathrm{H}-2$ & 94 & 95 & 0.70 & 0.02 & 0.0074 & 0.0005 & 0.062 & 0.000 & 0.17 & & 0.55 \\
\hline $6 \mathrm{H}-5$ & 17 & 18 & 0.80 & & 0.0064 & & & & 0.10 & & 0.24 \\
\hline $8 \mathrm{H}-5$ & 33 & 34 & 0.64 & 0.02 & 0.0049 & 0.0009 & 0.055 & & 0.20 & 0.04 & 0.18 \\
\hline $8 \mathrm{H}-5$ & 41 & 42 & 0.40 & & 0.0146 & & 0.039 & & 0.10 & & 0.29 \\
\hline $10 \mathrm{H}-6$ & 131 & 132 & 0.76 & & 0.0135 & & 0.047 & & 0.10 & & 0.18 \\
\hline $11 \mathrm{H}-2$ & 104 & 105 & 0.52 & & 0.0042 & & 0.020 & & 0.07 & 0.00 & 0.15 \\
\hline $11 \mathrm{H}-2$ & 107 & 108 & 0.48 & & 0.0043 & & 0.016 & & 0.04 & & 0.02 \\
\hline $11 \mathrm{H}-4$ & 90 & 91 & 0.58 & & 0.0129 & & 0.045 & & 0.17 & & 1.33 \\
\hline $12 \mathrm{H}-1$ & 118 & 119 & 0.36 & & 0.0123 & & 0.044 & & 0.16 & & 1.33 \\
\hline $12 \mathrm{H}-3$ & 127 & 128 & 0.82 & & 0.0067 & & 0.064 & & 0.11 & & 1.74 \\
\hline $12 \mathrm{H}-3$ & 130 & 131 & 1.26 & & 0.0079 & & 0.082 & & 0.11 & & 0.23 \\
\hline $12 \mathrm{H}-4$ & 21 & 22 & 0.66 & & 0.0098 & & 0.059 & & 0.17 & & 0.61 \\
\hline $12 \mathrm{H}-4$ & 39 & 40 & 0.77 & & 0.0124 & & 0.053 & & 0.07 & & 0.55 \\
\hline $13 \mathrm{H}-6$ & 139 & 140 & 0.34 & & 0.0040 & & 0.019 & & 0.04 & & 0.40 \\
\hline $13 \mathrm{H}-7$ & 44 & 45 & 0.32 & 0.01 & 0.0038 & 0.0002 & 0.017 & 0.000 & 0.04 & & 0.03 \\
\hline $14 \mathrm{H}-1$ & 49 & 50 & 1.35 & 0.03 & 0.0147 & 0.0004 & 0.085 & 0.001 & 0.13 & 0.02 & 0.28 \\
\hline $14 \mathrm{H}-3$ & 40 & 41 & 0.38 & & 0.0060 & & & & 0.25 & & 0.09 \\
\hline $14 \mathrm{H}-3$ & 87 & 88 & 0.39 & 0.00 & 0.0181 & 0.0000 & 0.044 & 0.001 & 0.12 & & 1.35 \\
\hline $14 \mathrm{H}-4$ & 145 & 146 & 0.63 & & 0.0088 & & 0.080 & & 0.18 & & 0.34 \\
\hline $15 \mathrm{H}-1$ & 20.5 & 21.5 & 0.86 & & 0.0157 & & 0.078 & & 0.09 & & 0.72 \\
\hline $15 \mathrm{H}-1$ & 73 & 74 & 0.94 & & 0.0112 & & 0.102 & & 0.08 & & 0.20 \\
\hline $15 \mathrm{H}-2$ & 11 & 12 & 1.06 & & 0.0142 & & 0.096 & & 0.13 & & 0.63 \\
\hline $15 \mathrm{H}-3$ & 58 & 59 & 0.68 & & 0.0144 & & 0.065 & & 0.15 & & 0.86 \\
\hline $15 \mathrm{H}-4$ & 40 & 41 & 1.03 & & 0.0112 & & 0.064 & & 0.05 & & 0.10 \\
\hline $15 \mathrm{H}-6$ & 1 & 2 & 0.70 & & 0.0264 & & 0.052 & & 0.15 & & 1.60 \\
\hline $15 \mathrm{H}-7$ & 54 & 55 & 0.58 & & 0.0067 & & 0.040 & & 0.07 & & 0.10 \\
\hline $15 \mathrm{H}-7$ & 64 & 65 & 0.44 & & 0.0100 & & & & 0.12 & & 0.23 \\
\hline
\end{tabular}

OC $=$ organic carbon, $\mathrm{TIC}=$ total inorganic carbon . 